UNITED STATES

DEPARTMENT OF THE INTERIOR

GEOLOGICAL SURVEY

\title{
DRAINAGE AREAS OF SELECTED SURFACE-WATER SITES IN FLORIDA
}

OPEN-FILE REPORT $\mathbf{8 0 - 9 5 7}$

Prepared in cooperation with

FLORIDA DEPARTMENT OF ENVIRONMENTAL REGULATION

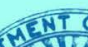


UNITED STATES

DEPARTMENT OF THE INTERIOR

GEOLOGICAL SURVEY

DRAINAGE AREAS OF SEILECTED SURFACE-WATER SITES IN FLORIDA

By Donald W. Foose

Open-File Report 80-957

Prepared in cooperation with

FLORIDA DEPARTMENT OF ENVIRONMENTAL REGULATION

Tallahassee, Florida

1980 
UNITED STATES DEPARTMENT OF THE INTERIOR

CECIL D. ANDRUS, Secretary

GEOLOGICAL SURVEY

H. William Menard, Director

For additional information write to:

U.S. Geological Survey

325 John Knox Road, Suite F-240

Tallahassee, Florida 32303 
Page

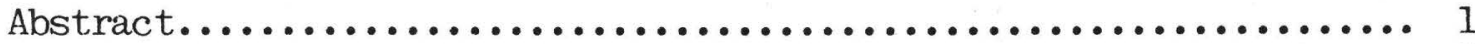

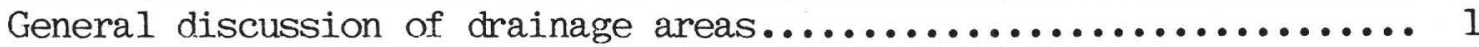

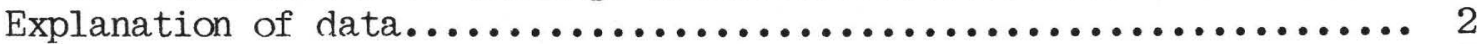

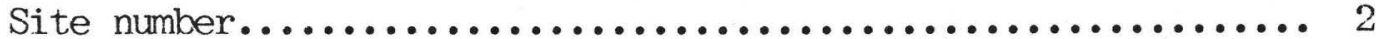

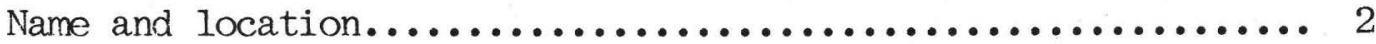

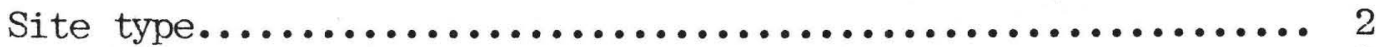

Drainage area and drainage area qualifier.................. 2

Surface area at elevation.............................. 4

Latitude - longitude................................ 4

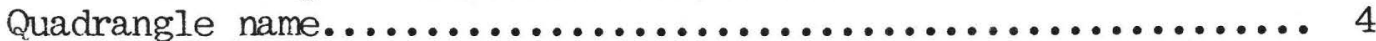

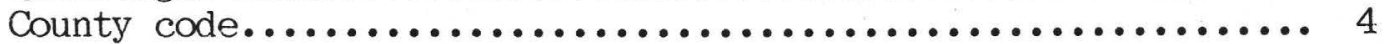

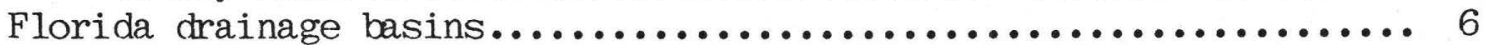

How drainage areas are determined.......................... 8

Drainage areas for selected stream sites in Florida.............. 11

St. Marys River basin............................... 12

Coastal area between St. Marys and St. Johns River........... 14

St. Johns River basin above Oklawaha River................. 14

Okalawaha River basin.............................. 19

St. Johns River basin below Oklawaha River................. 22

Coastal area between St. Johns River and Ponce de Leon

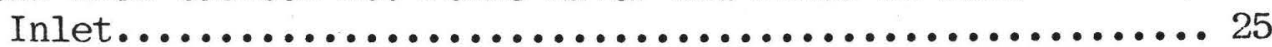

Coastal area between Ponce de Leon Inlet and Sebastian

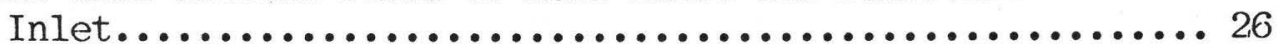

Coastal area Sebastian Inlet to St. Lucie River............. 26

Kissimee River basin ............................. 27

Taylor Creek basin and inflow to Lake Okeechobee from north.... 29

Fisheating Creek basin and inflow to Lake Okeechobee

from northwest............................... 30

Everglades and southeastern coastal area................ 30

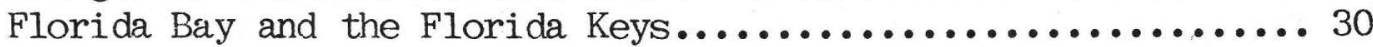

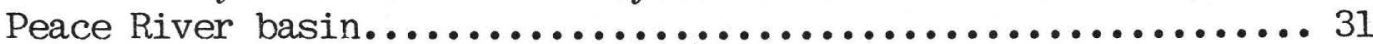

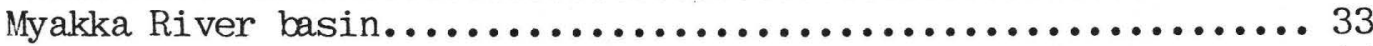

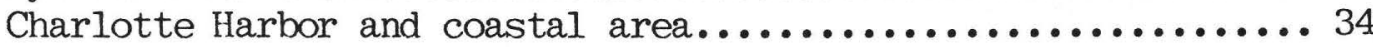

Coastal area between Myakka and Manatee Rivers............... 34

Manatee River basin................................... 35

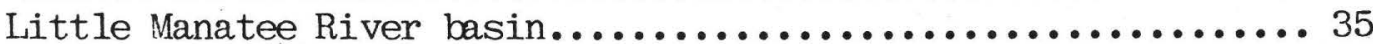

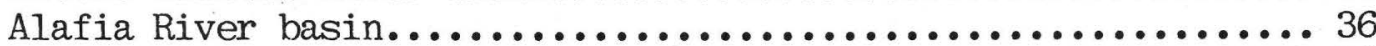

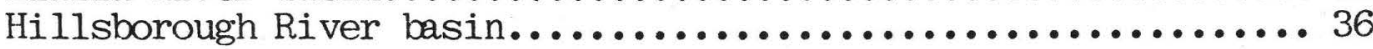

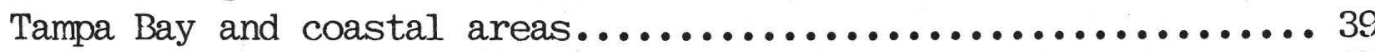

Coastal area from Tampa Bay to Withlacoochee River............ 40

Withlacoochee River basin........................... 42 
Drainage areas for selected stream sites in Florida--Continued Page Waccasassa River and coastal area between Withlacoochee

and Suwannee Rivers............................. 44

Coastal area between Suwannee and Aucilla Rivers.............. 44

Aucilla River basin.................................. 45

Suwannee River basin above Withlacoochee River

excluding Alapaha River basin.................... 45

Alapaha River basin.............................. 47

Withlacoochee River basin............................ 47

Suwannee River basin below Withlacoochee River

excluding Santa Fe River basin.................... 47

Santa Fe River basin............................... 48

St. Marks and Wakulla Rivers and coastal area between

Aucilla and Ochlockonee Rivers..................... 50

Ochlockonee River basin............................. 51

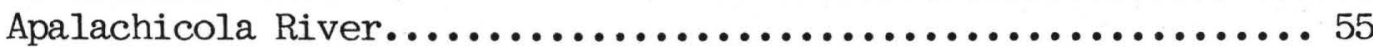

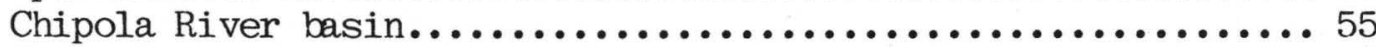

Coastal area between Ochlockonee and Apalachicola Rivers...... 56

St. Andrew Bay, inflow and coastal area................. 56

Choctawhatchee Bay, inflow and coastal area.............. 57

Yellow River basin................................ 58

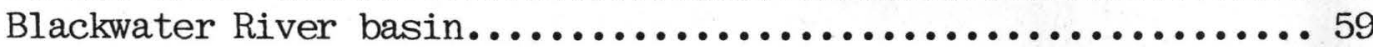

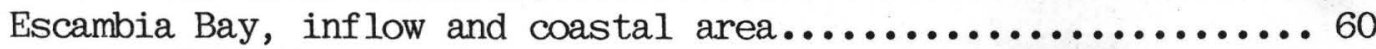

Perdido River basin................................ 62

Pea River......................................... 62

Choctawhatchee River below Pea River..................... 62

Escambia River..................................... 63

Drainage areas for selected lake sites in Florida............. 65

St. Marys River basin.............................. 66

St. Johns River above Oklawaha River.................... 66

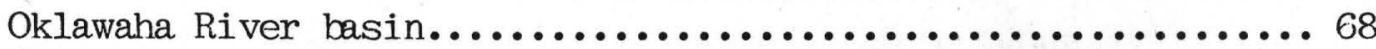

St. Johns River basin below Oklawaha River............... 70

Kissimee River basin............................... 70

Lake Okeechobee.................................... 72

Florida Bay and the Florida Keys........................ 72

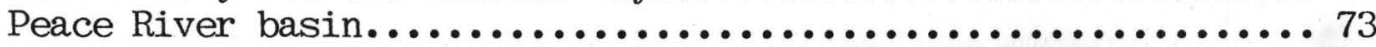

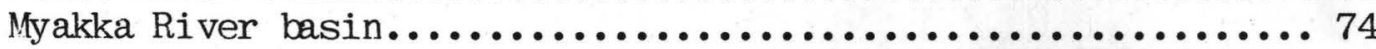

Little Manatee River basin............................ 75

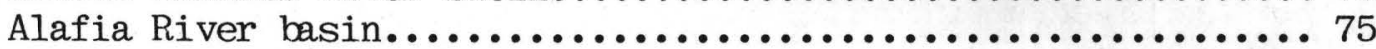

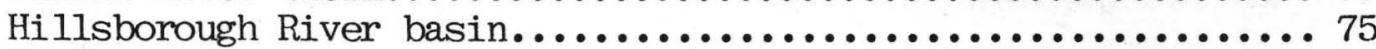

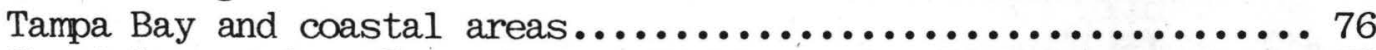

Coastal area from Tampa Bay to Withlacoochee River........... 78

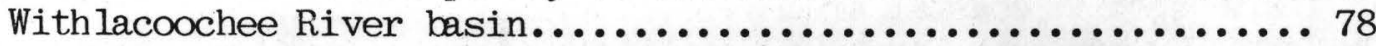

Wacasassa River and coastal area between Withlacoochee

and Suwannee Rivers............................. 79

Coastal area between Suwannee and Aucilla Rivers............. 79

Withlacoochee River basin........................... 80 
Drainage areas for selected lake sites in Florida--Continued Page Suwannee River basin below Withlacoochee River excluding Santa Fe River basin..................... 80

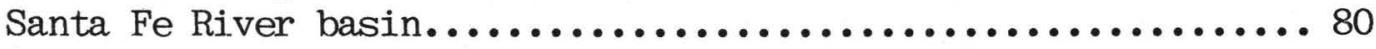

St. Marks and Wakulla Rivers and coastal area between

Aucilla and Ochlockonee Rivers..................... 80

Ochlockonee River basin............................ 81

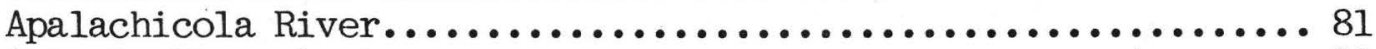

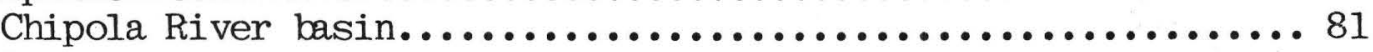

St. Andrew Bay, inflow and coastal area................. 82

Yellow River basin................................ 82

Pea River.......................................... 82

Choctawhatchee River below Pea River...................... 82

References......................................... 83

ILLUSTRATIONS

Page

Figure 1.--Example of drainage basin map................... 9

\section{TABLES}

Table 1.--Hydrologic unit names and numbers for region 03 in Florida................................... 3

2.-Codes for counties in Florida.................... 5

3.-Major stream systems in Florida................... 6 

DRAINAGE AREAS OF SELECTED SURFACE-WATER. SITES IN FLORIDA

By

Donald W. Foose

\section{ABSTRACT}

Data on drainage areas for about 1,500 surface water sites on streams and lakes in Florida are contained in this report. Sites are described in relation to a nearby city or town, and are located by county, by latitude-longitude, and by the U.S. Geological Survey 7 1/2-minute topographic map on which it is located. In addition, the surface areas of lakes are shown for the elevation given on the topographic map. These data were retrieved from the Surface Water Index developed and maintained by the Hydrologic Records Section of the Florida District Office, Water Resources Division of the U.S. Geological Survey.

\section{GENERAL DISCUSSION OF DRAINAGE AREAS}

A surface stream or a body of impounded surface water (a lake, pond, or swamp) together with all tributary surface streams and bodies of impounded surface water occupies a part of the Earth's surface known as a drainage basin. The drainage basin encloses the area up to the highest points between it and all other adjacent basins; a line connecting these points of highest elevation is called the basin boundary. The line is also the boundaries for the adjacent basins and when considered in that context is called the basin divide.

The basin divide is most often the natural crest of the area between the water features, but man-made structures such as dams, levees, dikes and road grades are designed to interrupt the flow of water and can become the basin divide. Canals, ditches, and culverts breach natural divides and can alter basin boundaries also.

The drainage boundary encloses an area from which surface runoff from precipitation normally drains by gravity into the stream and impounded surface water. This area is measured in a horizontal plane and defined as the drainage area for the basin. Drainage areas can also be determined for any point on a stream. A basin contained within a larger basin generally contributes its runoff to the larger basin. If it does not, it is called a closed or noncontributing basin. A basin may also be considered partially noncontributing when runoff stops below a certain elevation. The drainage areas in this report include non-contributing areas except when noted. 
The information for selected sites is separated into tabulations for streams and lakes, and listed by downstream order, hydrologic unit, subregion and cataloging units. See Table 1 for hydrologic unit names and numbers as adapted from Map Series No. 72. (Conover and Leach, 1975). An explanation of the information in each column follows:

Site Number.-The site number is the downstream order number for locations where data have been collected. Where no number is shown the location is most often the mouth of gaged streams but in some cases the location is where a stream crosses the state line or where a tributary stream enters the principal stream.

Name and Location.--The surface-water data collection sites in this report follow the procedure established by the U.S. Geological Survey for naming gaging stations. They are given names that reflect the specific and generic names of the water feature, an indicator of distance, and the name of a nearby city or town. To avoid confusion between sites that would ordinarily have the same name, a second more particular location is occasionally added for clarity.

Site Type (streams only).--Indicates the type of stream location for which the area has been determined. The codes are:

$$
\begin{aligned}
& \text { SW - Surface Water (natural stream with a defined channel) } \\
& \text { SL - Slough (natural stream without a defined channel) } \\
& \text { DR - Drain (a naturally occurring overflow channel) } \\
& \text { DD - Drainage Ditch (man-made overflow channel) } \\
& \text { RE - Reservoir ( man-made lake) } \\
& \text { SP - Spring } \\
& \text { OT - Other }
\end{aligned}
$$

Drainage area and drainage area qualifier.-The drainage area is shown in square miles. The precision is limited as explained in the discussion of how drainage areas are determined and where necessary is qualified as APPROX (approximate) or ABOUT. If noncontributing areas have been determined the qualifier NONCONTR is used.

The drainage area values in this report are rounded as follows:

0.01 square mile for areas less than 10 square miles;

0.1 square mile for areas 10 to 99.9 square miles;

1 square mile for areas 100 to 999 square miles.

Precision is affected by several circumstances. When maps other than 7 1/2-minute U.S. Geological Survey quadrangles are used, where the headwaters of two or more streams arise in the same swamp, where water is interchanged between basins, or where low relief is present, the resulting drainage area is rounded one more place and qualified approximate. For even less reliability, the results are rounded two (or more) places and qualified about. Less confidence than any of the above does not justify a basin houndary and the area is considered indeterminate. 
Table 1.-Hydrologic unit names and numbers for region 03 in Florida (Modified from Conover and Leach, 1975)

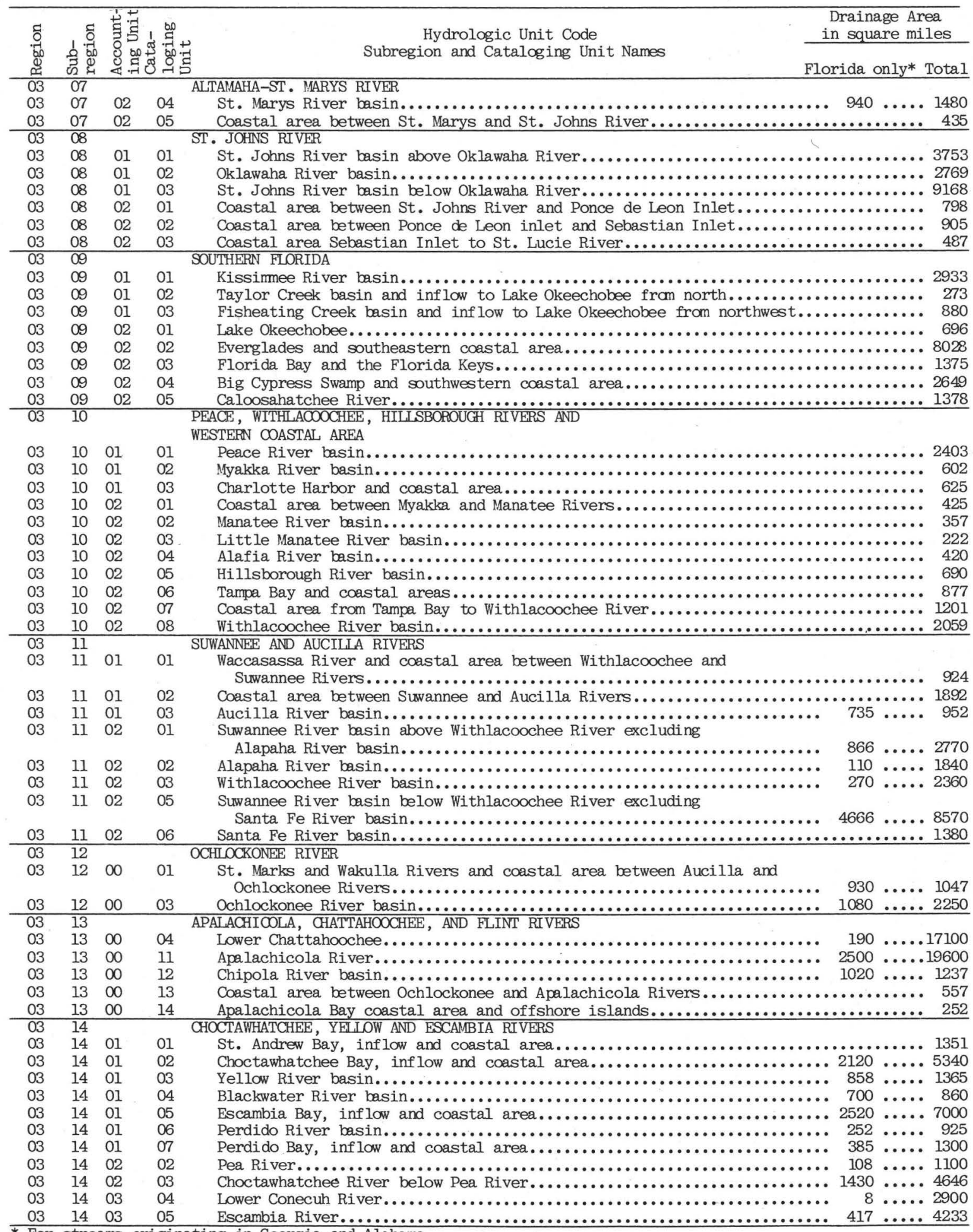

* For streams originating in Georgia and Alabama 
Surface Area at Elevation (lakes only).-The surface area of lakes is shown in acres as delineated on the $71 / 2$-minute topographic maps. It includes only the area bounded by the continuous darker blue shoreline and excludes islands. It is valid only for the elevation shown on the map.

Latitude-longitude.--The location of the site in degrees, minutes, and seconds.

Quadrangle name.--The name of the U.S. Geological Survey 7 1/2-minute topographic map on which the site is located.

County code.-The numerical code for Florida counties as outlined in the Bureau of Standards Publication FIPS-5-1 and 6-1. Table 2 lists the counties and codes. 
TABLE 2.--Codes for counties in Florida

\begin{tabular}{|c|c|c|c|}
\hline CODE & COUNTY & CODE & COUNTY \\
\hline 001 & ALACHUA & 067 & LAFAYETTE \\
\hline 003 & BAKER & 069 & LAKE \\
\hline 005 & BAY & 071 & LEE \\
\hline 007 & BRADFORD & 073 & LEON \\
\hline 009 & BREVARD & 075 & LEVY \\
\hline 011 & BROWARD & 077 & LIBERTY \\
\hline 013 & CALHOUN & 079 & MADISON \\
\hline 015 & CHARLOTTE & 081 & MANATEE \\
\hline 017 & CITRUS & 083 & MARION \\
\hline 019 & CLAY & 085 & MARTIN \\
\hline 021 & COLLIER & 087 & MONROE \\
\hline 023 & COLUMB IA & 089 & NASSAU \\
\hline 025 & DADE & 091 & OKALOOSA \\
\hline 027 & DE SOTO & 093 & OKEECHOBEE \\
\hline 029 & DIXIE & 095 & ORANGE \\
\hline 031 & DUVAL & 097 & OSCEOLA \\
\hline 033 & ESCAMBIA & 099 & PALM BEACH \\
\hline 035 & FLAGLER & 101 & PASCO \\
\hline 037 & FRANKLIN & 103 & PINELLAS \\
\hline 039 & GADSDEN & 105 & POLK \\
\hline 041 & GILCHRIST & 107 & PUTNAM \\
\hline 043 & GLADES & 109 & ST. JOHNS \\
\hline 045 & GULF & 111 & ST. LUCIE \\
\hline 047 & HAM ILTON & 113 & SANTA ROSA \\
\hline 049 & HARDEE & 115 & SARASOTA \\
\hline 051 & HENDRY & 117 & SEMINOLE \\
\hline 053 & HERNANDO & 119 & SUMTER \\
\hline 055 & HIGHLANDS & 121 & SUWANNEE \\
\hline 057 & HILLSBOROUGH & 123 & TAYLOR \\
\hline 059 & HOLMES & 125 & UNION \\
\hline 061 & INDIAN RIVER & 127 & VOLUSIA \\
\hline 063 & JACKSON & 129 & WAKULLA \\
\hline \multirow[t]{2}{*}{065} & JEFFERSON & 131 & WALTON \\
\hline & & 133 & WASHINGTON \\
\hline
\end{tabular}




\section{FLORIDA DRAINAGE BASINS}

Florida has 30 stream systems that have a major impact because of their size or location. Table 3 lists these streams and the bodies of water which receives runoff from them.

TABLE 3.-Major stream systems in Florida

\begin{tabular}{|c|c|}
\hline STREAM & OUTFALL \\
\hline St. Mary River & Cumberland Sound \\
\hline St. Johns River & Atlantic Ocean \\
\hline Oklawaha River & St. Johns River \\
\hline Kissimmee River & Lake Okeechobee \\
\hline Taylor Creek & Lake Okeechobee \\
\hline Fisheating Creek & Lake Okeechobee \\
\hline Lake Okeechobee outf low & Bays - Atlantic Ocean to Gulf of Mexico \\
\hline Peace River & Charlotte Harbor \\
\hline Myakka River & Charlotte Harbor \\
\hline Manatee River & Tampa Bay \\
\hline Little Manatee River & Tampa Bay \\
\hline Alafia River & Hillsborough Bay \\
\hline Hillsborough River & Tampa Bay \\
\hline Withlacoochee Piver & Gulf of Mexico (Withlacoochee Bay) \\
\hline Waccasassa River & Gulf of Mexico (Waccasassa Bay) \\
\hline Suwannee River & Gulf of Mexico \\
\hline Alapaha River & Suwannee River \\
\hline Withlacoochee River & Suwannee River \\
\hline Santa Fe River & Suwannee River \\
\hline Aucilla River & Gulf of Mexico \\
\hline St. Marks River & Gulf of Mexico (Apalachee Bay) \\
\hline Ochlockonee River & Ochlockonee Bay \\
\hline Apalachicola River & Apalachicola Bay \\
\hline Chipola River & Apalachicola River \\
\hline Yellow River & Blackwater Bay \\
\hline Shoal River & Yellow River \\
\hline Blackwater River & Blackwater Bay \\
\hline Choctawhatchee River & Choctawhatchee Bay \\
\hline Escambia River & Escambia Bay \\
\hline Perdido River & Perdido Bay \\
\hline
\end{tabular}

Only one of the streams in table 3 terminates in the Atlantic Ocean. Six terminate in other streams, three terminate in Lake Okeechobee, five terminate in the Gulf of Mexico, and the rest terminate in bays.

A system of classification called hydrologic units (see table 1) has boundaries based on basin divides with some important exceptions: notably the Florida gulf coast bays, Florida Bay; the Big Cypress Swamp; and Lake Okeechobee.

Because of the low relief on most of peninsular Florida, hydrologic divides are often difficult to delineate. 
Water flow over much of southeast Florida is controlled by canals, dikes, storage in impoundments, and other man-made control devices so that natural divides do not apply and drainage areas are meaningless. The drainage pattern of some streams such as the Caloosahatchee and Kissimmee Rivers and Reedy and Shingle Creeks have been so changed by man-made structures that the drainage areas as formerly determined have been revised to "indeterminate."

Springs and canals would not ordinarily have a meaningful drainage area. Some canals are "improved" streams and retain some stream characteristics. A good example is Sumter Creek Canal in west-central Florida. Drainage areas with appropriate qualifiers can then be determined.

There are two significantly noncontributing areas in Florida: Approximately 50 square miles near Bennett in northern Florida called the Deadenings and approximately 650 square miles near Gainesville in central Florida called Paynes Prairie. Numerous other minor noncontributing areas, such as sinkholes exist, however. Many lakes and ponds have no perennial outlets and have either noncontributing or partially noncontributing areas.

Many Florida river systems have their headwaters in swamps. The St. Johns River for example, flows out of the St. Johns Marsh which extends into St. Lucie County. The St. Marys and Suwannee Rivers originate in the extensive Okefenokee Swamp of southern Georgia. The Econfina, Fenholloway and Steinhatchee Rivers, and Spring Warrior Creek originate in San Pedro Bay. The Green Swamp is the headwaters of three major systems, the Withlacoochee, the Oklawaha, and the Hillsborough. When more than one stream originates in a swamp, the drainage area for the swamp, in the absence of field data to the contrary, is split midway between the channels of the affected streams.

Several streams in Florida disappear underground. The Aucilla River in Jefferson County, Lost Creek and the St. Marks River in Wakulla County, Little River in Suwannee County, Falling Creek and Rose Creek in Columbia County, the Alapaha River in Madison County, the Little Alapaha River in Hamilton County, Norton Creek in Madison County, and the Santa Fe River in Alachua County among others. The drainage area for these streams is sometimes considered a closed basin and noncontributing to any other streams, but is sometimes considered to be contributing to another stream, based on the analysis of the situation for that stream. 
Drainage basin boundaries and divides for the Florida District are determined in conformity to Federal Interagency River Basin Committee, Notes on Hydrologic Activities-Bulletin No. 4, November 1951: "InterAgency Coordination of Drainage Area Data", which establishes both standards and methodology. U.S. Geological Survey 7 1/2-minute maps are the maps on which most drainage area delineations are made. When $71 / 2-$ minute topographic maps are not available state base maps, air photomaps, county maps, or state highway survey maps are used. As of 1979, the entire State of Florida is mapped on 1047, 7 1/2-minute, 1:24000 scale, U.S. Geological Survey topographic maps. These maps are considered to be accurate within 40 feet horizontally and one-half the contour interval vertically.

In order to determine a drainage area, a boundary is delineated along the natural ridge or the top of man-made features as shown on the map of the area. The effect of man-made drainage is taken into account. In some cases an on-the-ground investigation is made. The portion of the area for each site on each map is planimetered, or measured by a mechanical or electronic device, which relates the area inside a closed line on the map to an area on the ground. Each map represents an area on the Earth's surface as determined on Smithsonian geological tables. When the total as planimetered is different from the area in the table, a constant is determined and each map area multiplied by it to balance the map.

After all the maps for a basin or site have been planimetered and the areas compared to and balanced against the area of the quadrangle, the areas for each site or basin are added together and a drainage area in square miles is tabulated.

The Florida district office of the U.S. Geological Survey maintains a file of maps on which drainage areas are determined. In 1978, in cooperation with the Florida Department of Environmental Regulation, the District traced the entire file onto the latest 1:24000-scale 7 1/2-minute topographic maps using a unified symbology. See figure 1 for an example of the basin map.

The major basin boundaries are the heavy lines and generally correspond with the hydrologic unit lines. Basins for tributaries to the major streams, and basins for coastal streams in coastal hydrologic units are outlined in the second heaviest lines; basins for tertiary streams are outlined in the third heaviest lines; and basins for other minor streams or landlocked lakes are outlined in the thinest lines. Drainage areas for points along streams, such as gaging stations, lake outlets, or dams are shown by dashed lines. Block symbols with a number refer to locations where surface-water data have been or are being collected. Block symbols with a dot in the center indicate miscellaneous measurements at sites not operated on a periodic basis. Circles are sites where water-quality data were collected without discharge or lake elevation information. 
UNITED STATES
G. DEFARTMNT OF THE INTERIOR
GEOLOGICAL SURVEY

ORI ANDO WEST QUADRANGLE

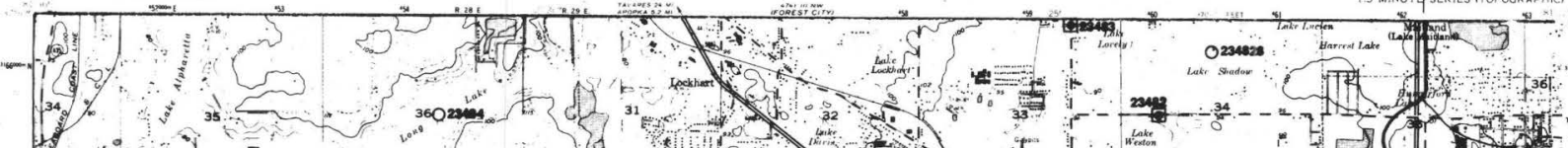

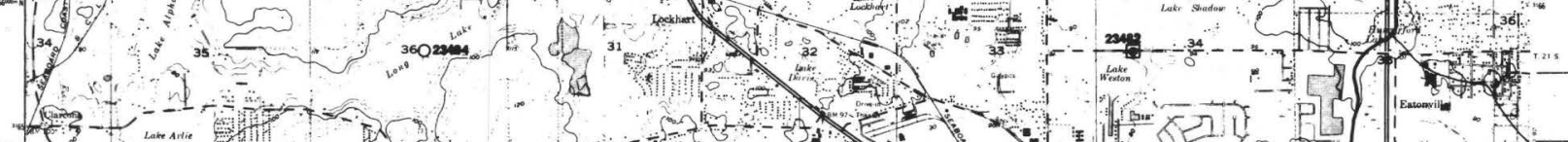

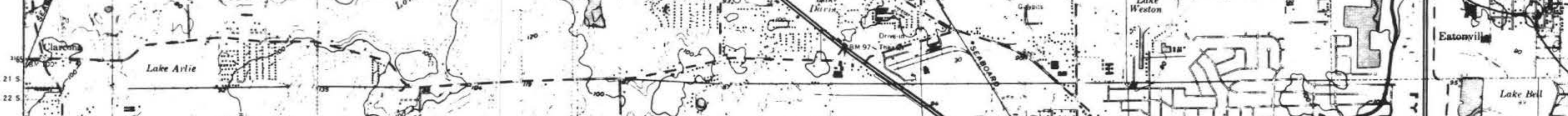

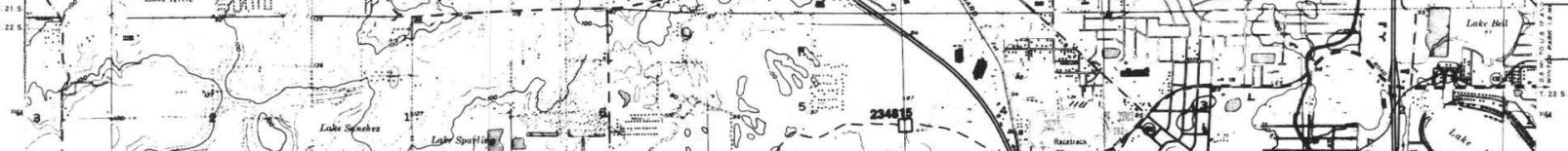

(1)

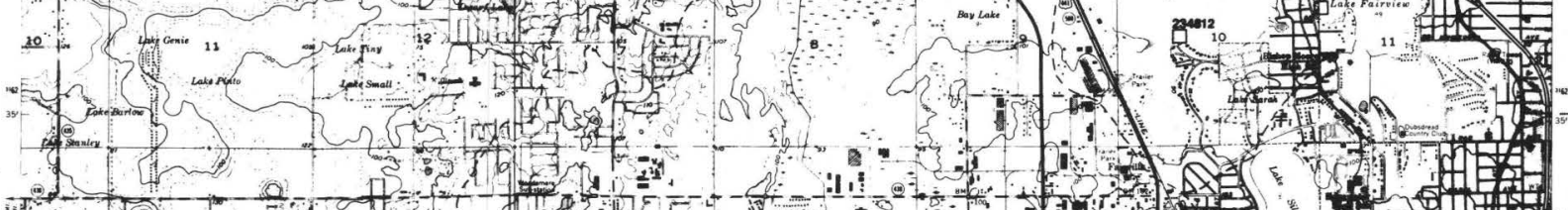

(150)

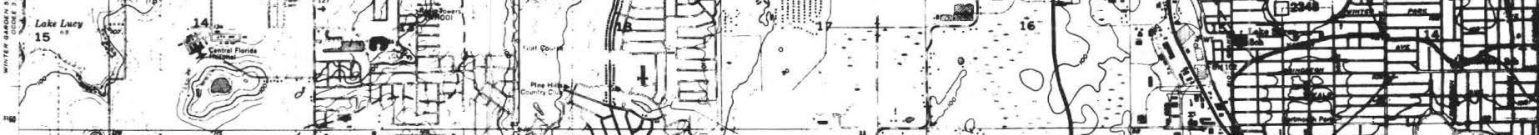

- 0 1.

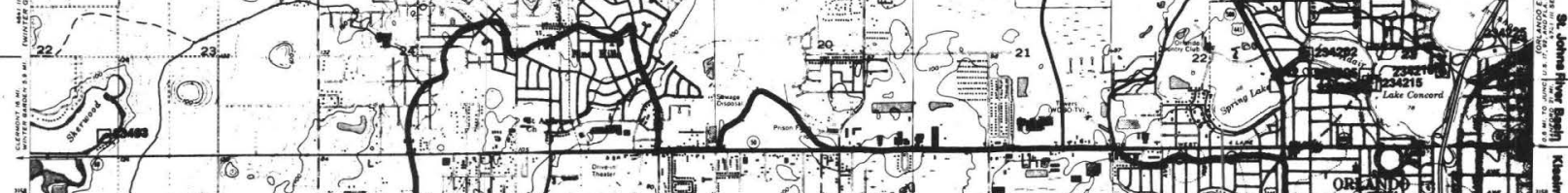

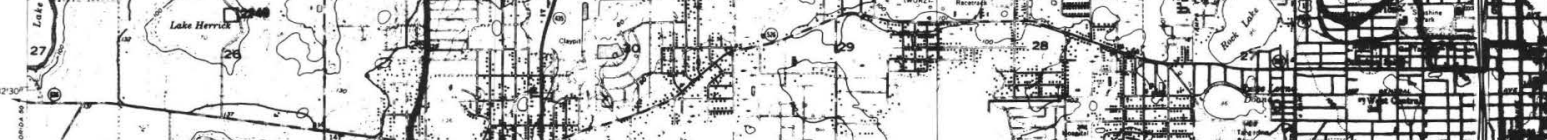
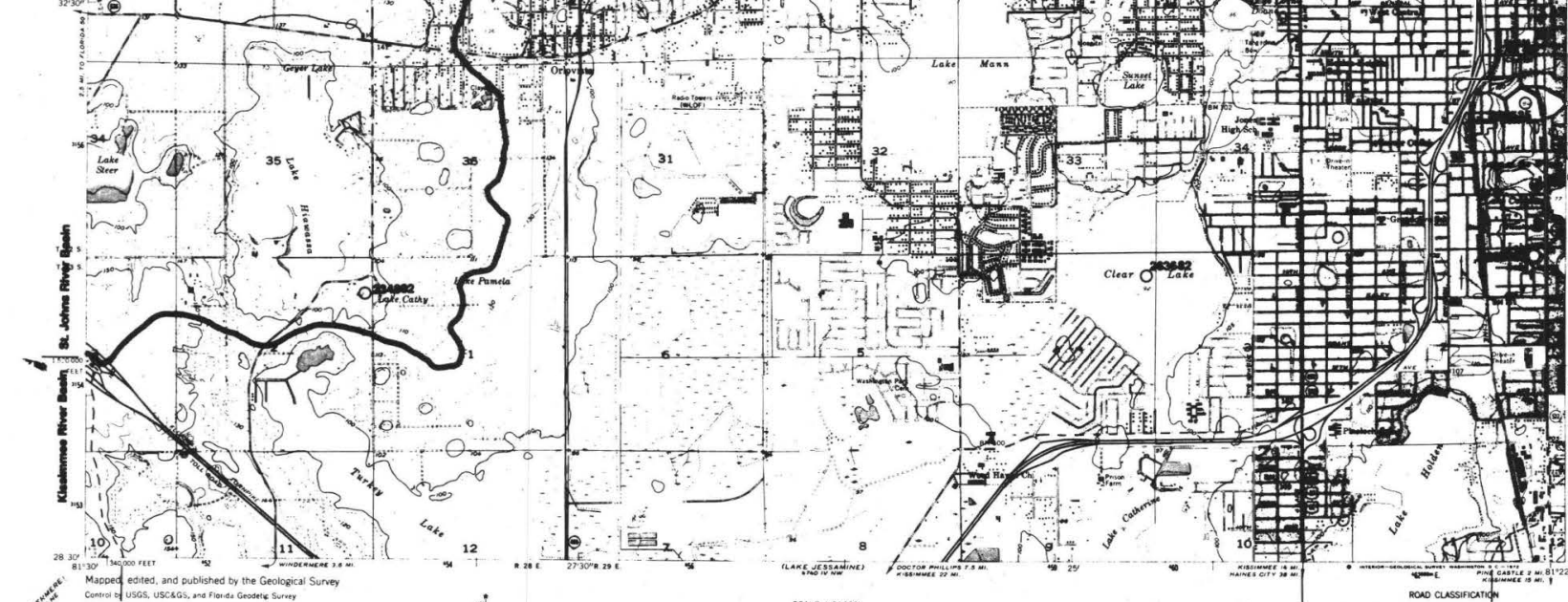

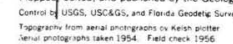

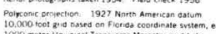

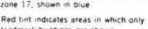
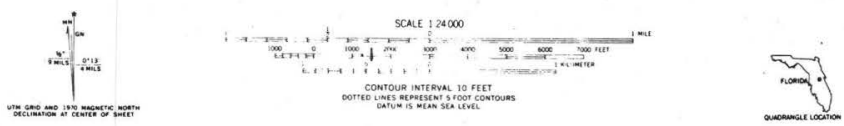

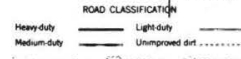

Figure 1. Example of drainage basin map. 
Ozalid copies of these maps are available from the U.S. Geological Survey, 325 John Knox Road, Suite F-240, Tallahassee, Florida 32303 for the cost of reproduction. An Index to Topographic Maps of Florida, issued by the Topographic Division of the U.S. Geological Survey is contained in the back of this report. The name of each quadrangle is shown on the Index and is the only identification for ordering copies. 
DRAINAGE AREAS FOR SEILECTED STREAM SITES IN FLORIDA 
SI TE

NUMBER

NAME AND LOCATION

02228500 NORTH PRONG ST MARYS RIVER AT MONIAC GA ST MARYS RIVER BASIN IN GEORGIA (TOTAL) 02229000 MIDDLE PRONG ST MARYS RIVER AT TAYLOR MIDDLE PRONG ST MARYS RIVER AT MOUTH

02229300 CEDAR CREEK NR GLEN ST MARY CEDAR CREEK AT MOUTH

02229500 SOUTH PRONG ST MARYS RIVER NR SANDERSON 02230000 TURKEY CREEK AT MACCLENNY TURKEY CREEK AT MOUTH

02230500 SOUTH PRONG ST MARYS RIVER AT GLEN ST MARY SOUTH PRONG ST MARYS RIVER AT MOUTH

02231000 ST MARYS RIVER NR MACCLENNY

02231050 DEEP CREEK NR RALWIN

02231051 DEEP CREEK TRIBUTARY NO 3 NR BALDWIN 02231052 DEEP CREEK TRIBUTARY NR BALDWIN

02231054 DEEP CREEK TRIBUTARY NO 2 NR BALDWIN DEEP CREEK AT MOUTH

02231065 BRANDY BRANCH NR BRYCEVILLE BRANDY BRANCH AT MOUTH

02231070 ST MARYS RIVER TRIBUTARY NR BRYCEVILLE 02231080 DEEP CREEK NO 2 NR BRYCEVILLE

DEEP CREEK NO 2 AT MOUTH

02231085 ST MARYS RIVER TRIBUTARY NO 2 NR KENT 02231090 ST MARYS RIVER TRIBUTARY NO 3 NR KENT 02231100 ST MARYS RIVER NR ST GEORGE GA

\begin{tabular}{|c|c|c|c|c|c|c|c|}
\hline $\begin{array}{l}\text { SITE } \\
\text { TYPE }\end{array}$ & $\begin{array}{l}\text { DRAINAGE } \\
\text { AREA } \\
\text { SQ.MI. }\end{array}$ & $\begin{array}{l}\text { DRAINAGE } \\
\text { AREA } \\
\text { QUAL IF IER }\end{array}$ & $\begin{array}{c}\text { LAT } \\
\mathrm{DG} / \mathrm{MI} / \mathrm{SO}\end{array}$ & $\begin{array}{c}\text { LONG } \\
\mathrm{DG} / \mathrm{MI} / \mathrm{SD}\end{array}$ & QUADRANGL & LE NAME & $\begin{array}{l}\text { CNTY } \\
\text { CODE }\end{array}$ \\
\hline SW & 160 & APPROX & $30 / 31 / 03$ & $82 / 13 / 50$ & MONIAC & & 003 \\
\hline SW & 540 & APPROX & & & & & \\
\hline SW & 125 & & $30 / 26 / 10$ & $82 / 17 / 20$ & TAYLOR & & 003 \\
\hline SW & 174 & & $30 / 25 / 25$ & $82 / 12 / 35$ & MACCLENNY & NW & \\
\hline SW & 71.4 & & $30 / 20 / 20$ & $82 / 12 / 00$ & MACCLENNY & WEST & 003 \\
\hline SW & 80.0 & & $30 / 21 / 38$ & $82 / 10 / 23$ & MACCLENNY & WEST & \\
\hline SW & 57.8 & APPROX & $30 / 12 / 17$ & $82 / 15 / 49$ & SANDERSON & SOUTH & 003 \\
\hline SW & 19.9 & & $30 / 16 / 05$ & $82 / 07 / 20$ & MACCLENNY & EAST & 003 \\
\hline SW & 27.0 & & $30 / 15 / 53$ & $82 / 09 / 02$ & MACCLENNY & WEST & \\
\hline SW & 156 & & $30 / 16 / 40$ & $82 / 08 / 40$ & MACCLENNY & WEST & 003 \\
\hline SW & 183 & & $30 / 21 / 46$ & $82 / 06 / 03$ & MACCLENNY & EAST & \\
\hline SW & 700 & APPROX & $30 / 21 / 35$ & $82 / 04 / 55$ & MACCLENNY & EAST & 003 \\
\hline SW & $23 . \bar{c}$ & & $30 / 18 / 02$ & $82 / 01 / 50$ & MACCLENNY & EAST & 089 \\
\hline SW & 1.89 & & $30 / 18 / 30$ & $82 / 00 / 30$ & MACCLENNY & EAST & 089 \\
\hline SW & .53 & & $30 / 18 / 50$ & $82 / 00 / 50$ & MACCLENNY & EAST & 089 \\
\hline SW & 6.71 & & $30 / 19 / 30$ & $82 / 00 / 30$ & MACCLENNY & EAST & 031 \\
\hline SW & 48.5 & & $30 / 21 / 56$ & $82 / 02 / 31$ & MACCLENNY & EAST & \\
\hline SW & 62.5 & & $30 / 22 / 00$ & $82 / 01 / 00$ & MACCLENNY & EAST & 089 \\
\hline Sw & 66.9 & & $30 / 22 / 45$ & $82 / 02 / 16$ & MACCLENNY & NE & \\
\hline SW & 1.67 & & $30 / 24 / 20$ & $82 / 01 / 50$ & MACCLENNY & NE & 089 \\
\hline SW & 10.1 & & $30 / 27 / 20$ & $82 / 00 / 20$ & MACCLENNY & NE & 089 \\
\hline SW & 14.7 & & $30 / 27 / 37$ & $82 / 01 / 24$ & MACCLENNY & NE & \\
\hline SW & 3.29 & & $30 / 28 / 52$ & $81 / 59 / 55$ & BRYCEVILLE & & 089 \\
\hline SW & 1.7 & & $30 / 31 / 00$ & $81 / 59 / 30$ & HILLIARD S & SW & 089 \\
\hline SW & 890 & APPROX & $30 / 31 / 28$ & $82 / 01 / 07$ & ST GEORGE & & 089 \\
\hline
\end{tabular}


DRAINAGE AREAS FOR SELECTED STREAM SITES IN FLORIDA

AL TAMAHA/ST. MARYS RIVERS

ST. MARYS RIVER BASIN

SITE
NUMBER

NAME AND LOCATION

02231105 ST MARYS RIVER TRIBUTARY NO 4 NR KENT

02231110 MILL CREEK NR KENT

MILL CREEK AT MOUTH

02231120 DEEP CREEK NO 3 NEAR KENT

DEEP CREEK NO 3 AT MOUTH

02231130 STAVE BRANCH NR HILLIARD

STAVE BRANCH AT MOUTH

02231145 DUNN CREEK NR BOULOGNE

DUNN CREEK AT MOUTH

02231150 LITTLE DUNN CREEK NR BOULOGNE

LITTLE DUNN CREEK AT MOUTH

02231170 BRUSH CREEK NR BOULOGNE

BRUSH (BRUSHY) CREEK AT MOUTH

02231220 ST MARYS RIVER AT BOULOGNE

02231230 PIGEON CREEK AT BOULOGNE

PIGEON CREEK AT MOUTH

02231238 ST MARYS RIVER TRIBUTARY AT KINGS FERRY

02231242 CABBAGE CREEK NR KINGS FERRY

CABBAGE CREEK AT MOUTH

02231250 LITTLE ST MARYS RIVER NR HILLIARD

02231251 LITTLE ST MARYS RIVER AT LESSIE

02231252 WILDER CREEK AT LESSIE

LITTLE ST MARYS RIVER AT MOUTH

02231253 ST MARYS RIVER NR GROSS

ST MARYS RIVER AT MOUTH

\begin{tabular}{|c|c|c|c|c|c|c|c|}
\hline $\begin{array}{l}\text { SITE } \\
\text { TYPE }\end{array}$ & $\begin{array}{l}\text { DRAINAGE } \\
\text { AREA } \\
\text { SQ.MI. }\end{array}$ & $\begin{array}{l}\text { DRAINAGE } \\
\text { AREA } \\
\text { QUALIFIER }\end{array}$ & $\begin{array}{c}\text { LAT } \\
D G / M I / S O\end{array}$ & $\begin{array}{c}\text { LONG } \\
\mathrm{DG} / \mathrm{MI} / \mathrm{SO}\end{array}$ & QUADRANGLE & NAME & $\begin{array}{l}\text { CNTY } \\
\text { CODE }\end{array}$ \\
\hline SW & 1.11 & & $30 / 32 / 30$ & $81 / 59 / 50$ & HILLIARD SW & & 089 \\
\hline SW & 31.0 & & $30 / 34 / 00$ & $81 / 59 / 30$ & HILLIARD SW & & 089 \\
\hline SW & 31.4 & & $30 / 33 / 58$ & $82 / 00 / 22$ & ST GEORGE & & \\
\hline SW & 11.2 & & $30 / 36 / 00$ & $82 / 00 / 00$ & HILLIARD SW & & 089 \\
\hline SW & 14.2 & & $30 / 36 / 14$ & $82 / 01 / 14$ & ST GEORGE & & \\
\hline SW & .28 & & $30 / 37 / 44$ & $82 / 00 / 46$ & TOLEDO & & 089 \\
\hline SW & 1.15 & & $30 / 36 / 22$ & $82 / 01 / 24$ & ST GEORGE & & \\
\hline SW & 9.91 & & $30 / 42 / 26$ & $82 / 01 / 19$ & TOLEDO & & 089 \\
\hline SW & 10.7 & & $30 / 42 / 34$ & $82 / 02 / 11$ & TOLEDO & & \\
\hline SW & 8.72 & & $30 / 43 / 00$ & $82 / 00 / 26$ & TOLEDO & & 089 \\
\hline SW & 12.2 & & $30 / 45 / 02$ & $82 / 01 / 39$ & FOLKSTON & & \\
\hline SW & .85 & & $30 / 43 / 50$ & $81 / 59 / 40$ & HILL I ARD & & 089 \\
\hline SW & 4.02 & & $30 / 45 / 39$ & $82 / 00 / 44$ & FOLKSTON & & \\
\hline SW & 1,170 & APPROX & $30 / 46 / 30$ & $81 / 58 / 40$ & BOULOGNE & & 089 \\
\hline SW & 9.36 & & $30 / 45 / 39$ & $81 / 58 / 04$ & BOULOUGNE & & 089 \\
\hline SW & 11.9 & & $30 / 47 / 03$ & $81 / 58 / 14$ & BOUL OGNE & & \\
\hline SW & .16 & & $30 / 46 / 40$ & $81 / 50 / 50$ & KINGS FERRY & & 089 \\
\hline SW & 2.69 & & $30 / 45 / 10$ & $81 / 51 / 30$ & KINGS FERRY & & 089 \\
\hline SW & 9.63 & & $30 / 46 / 50$ & $81 / 47 / 50$ & KINGS FERRY & & \\
\hline SW & 19.8 & & $30 / 43 / 55$ & $81 / 53 / 35$ & HILL I ARD & & 089 \\
\hline SW & 39.5 & & $30 / 42 / 30$ & $81 / 46 / 40$ & HILLIARD NE & & 089 \\
\hline SW & 7.91 & & $30 / 42 / 40$ & $81 / 46 / 40$ & HILLIARD NE & & 089 \\
\hline SW & 64.4 & & $30 / 44 / 48$ & $81 / 42 / 42$ & GROSS & & \\
\hline SW & 1,360 & APPROX & $30 / 44 / 29$ & $81 / 41 / 17$ & GROSS & & 089 \\
\hline SW & 1,480 & APPROX & $30 / 43 / 29$ & $81 / 30 / 04$ & FERNANDINA & BEACH & \\
\hline
\end{tabular}


DRAINAGE AREAS FOR SELECTED STREAM SITES IN FLORIDA
ALTAMAHA/ST. MARYS RIVERS

COASTAL. AREA BETWEEN ST. MARYS AND ST. JOHNS RIVER

SITE
NUMBER

NAME AND LOCATION

02231260 MILLS CREEK NR HILLARD

02231263 MILLS CREEK NR CALLAHAN

02231267 SPELL SWAMP NR ITALIA

SPELL SWAMP AT MOUTH

02231270 ALLIGATOR CREEK NR CALLAHAN

02231272 CUSHING CREEK AT CALLAHAN

CUSHING CREEK AT MOUTH

02231275 PLUMMER CREEK NR ITALIA

PLUMMER CREEK AT MOUTH

02231280 THOMAS CREEK NR CRAWFORO

02231282 THOMAS CREEK NR CALLAHAN

02231284 THOMAS CREEK AT STATE HIGHWAY 115 NR CALLAHAN

THOMAS CREEK AT MOUTH

02231290 LOFTON CREEK NR YULEE

NASSAU RIVER AT MOUTH

\begin{tabular}{|c|c|c|c|c|c|c|c|}
\hline $\begin{array}{l}\text { SITE } \\
\text { TYPE }\end{array}$ & $\begin{array}{l}\text { ORAINAGE } \\
\text { AREA } \\
\text { SQ.MI. }\end{array}$ & $\begin{array}{l}\text { DRAINAGE } \\
\text { AREA } \\
\text { QUALIF IER }\end{array}$ & $\begin{array}{c}\text { LAT } \\
D G / M I / S D\end{array}$ & $\begin{array}{c}\text { LONG } \\
\mathrm{DG} / \mathrm{MI} / \mathrm{SD}\end{array}$ & QUADRANGLE & NAME & $\begin{array}{l}\text { CNTY } \\
\text { CODE }\end{array}$ \\
\hline OT & 17.6 & & $30 / 38 / 21$ & $81 / 51 / 47$ & HILLI I ARD NE & & 089 \\
\hline SW & 37.9 & & $30 / 37 / 50$ & $81 / 47 / 40$ & HILLIARD NE & & 089 \\
\hline SW & 12.3 & & $30 / 38 / 10$ & $81 / 45 / 30$ & HILLIARD NE & & 089 \\
\hline SW & 13.0 & & $30 / 37 / 20$ & $81 / 45 / 15$ & CALLAHAN & & - \\
\hline SW & 25.8 & & $30 / 34 / 32$ & $81 / 49 / 10$ & CALLAHAN & & 089 \\
\hline SW & 19.9 & & $30 / 33 / 30$ & $81 / 49 / 10$ & CALLAHAN & & 089 \\
\hline SW & 22.0 & & $30 / 34 / 20$ & $81 / 49 / 05$ & HILLARD SW & & \\
\hline SW & 11.3 & & $30 / 37 / 53$ & $80 / 41 / 06$ & GROSS & & 089 \\
\hline SW & 24.3 & & $30 / 34 / 57$ & $81 / 39 / 24$ & I TAL I A & & \\
\hline SW & 29.9 & & $30 / 27 / 39$ & $81 / 49 / 57$ & DINSMORE & & 031 \\
\hline SW & 52.6 & & $30 / 30 / 20$ & $81 / 47 / 30$ & CALLAHAN & & 089 \\
\hline SW & 64.1 & & $30 / 31 / 00$ & $81 / 45 / 50$ & CALLAHAN & & 089 \\
\hline SW & 109 & & & & ITAL I A & & 089 \\
\hline SW & 400 & & & & AMELIA CITY & & 089 \\
\hline S & 221 & & $28 / 44 / 39$ & $81 / 52 / 22$ & HOWEY IN THE & HILL & 069 \\
\hline
\end{tabular}

ST. JOHNS RIVER

ST. JOHNS RIVER RASIN AROVE OKLAWAHA RIVER

SITE
NUMBER

NAME AND LOCATION

02231313 JOE GORE SLOUGH AT HILOLO

02231315 GOMEZ CREEK AT HILOLO

02231323 FORT DRUM CREEK AT US HIGHWAY 441 AT FORT DRUM

02231329 FORT DRUM CREEK AT FORT DRUM

02231333 FORT DRUM CREEK NR FORT ORUM

02231337 SWEETWATER BRANCH AT US HIGHWAY 441 NR FORT DRU

02231339 PARKER SLOUGH NR FORT DRUM
DRAINAGE DRAINAGE

SITE AREA AREA LAT LONG

TYPE. SO.MI. QUALIFIER DG/MI/SO DG/MI/SD QUADRANGLE NAME CODE

SL -9.24

27/26/56 80/46/32 TAYLOR CREEK NE

093

SW $\quad 14.5$

SW $\quad 18.4$

27/26/56 80/45/32 TAYLOR CREEK NE

$27 / 30 / 4580 / 48 / 26$ FORT DRUM

093

SW 25.3

27/31/22 80/47/56 FORT DRUM

093

SW 28.4

$27 / 32 / 50 \quad 80 / 47 / 59$ FORT DRUM

093

SW $\quad 3.48$

$27 / 34 / 1780 / 49 / 57$ FORT DRUM

093

SL $\quad 4.65$

27/35/04 80/50/19 FORT DRUM
093

093 
DRAINAGE AREAS FOR SELECTED STREAM SITES IN FLORIDA

ST. JOHNS RIVER BASIN ABOVE OKLAWAHA RIVER

SITE

NAME AND LOCATION

02231340 SWEETWATER BRANCH NR FORT DRUM

02231341 BOGGY BRANCH NR FT DRUM

02231342 FORT DRUM CREEK AT SUNSHINE ST PKWY NR FT DRUM 02231345 JIM GREEN CREEK NR FORT DRUM

02231350 ST JOHNS HEADWATERS NR VERO BEACH

02231380 BLUE CYPRESS CREEK NR YEHAW JUNCTION

02231390 COW LOG BRANCH NR YEEHAW JUNCTION

02231396 BLUE CYPRESS CREEK NR FELLSMERE

BLUE CYPRESS CREEK AT MOUTH
02231355 PADGETT BRANCH NR OSOWAW

\section{DRAINAGE DRAINAGE}

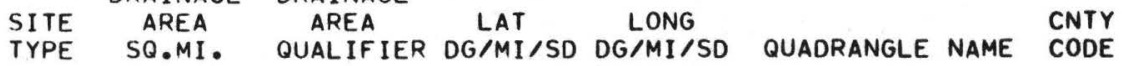

SW $9.82 \quad 27 / 33 / 58 \quad 80 / 49 / 03$ FORT DRUM 093

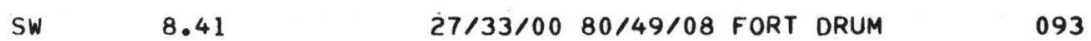

SW $\quad 52.6 \quad 27 / 34 / 0680 / 47 / 47$ FORT DRUM 093

SW $12.8 \quad 27 / 35 / 2280 / 48 / 28$ FORT DRUM 093

CN $29727 / 41 / 3280 / 25 / 00$ FELLSMERE 4 NW 061

SW $6.38 \quad 27 / 38 / 4280 / 52 / 37$ FORT DRUM NW 093

SW $59.8 \quad 27 / 54 / 4180 / 53 / 20$ FORT DRUM NW 097

SW $20.5 \quad 27 / 41 / 1980 / 52 / 52$ FORT DRUM NW 097

SW $105 \quad 27 / 43 / 4080 / 48 / 19$ FORT DRUM NE 061

SW $\quad 108$

CN $\quad 537$

02231460 ST JOHNS RIVER CREST STAGE GAGE NO I NR MICCO

02231467 ST JOHNS RIVER AB LAKE HELEN BLAZES NR MELBORNE 02231470 ST JOHNS RIVER CREST STAGE GAGE NO 2 NR MALABAR 02231475 BULL CREEK NR HOLOPAW

02231489 TYSON CREEK NR KENANSVILLE

02231496 PADGETT BRANCH NR KENANSVILLE

02231565 CRABGRASS CREEK NEAR HOLOPAW

02231600 JANE GREEN CREEK NR DEER PARK JANE GREEN CRFEK AT MOUTH

02231800 ST JOHNS RIVER CREST STAGE GAGE NO 3 NR MELBOUR 02232000 ST JOHNS RIVER NR MELBOURNE

02232120 ST JOHNS RIVER CREST STAGE NO 3A NR EAU GALLIE 02232140 ST JOHNS RIVER CREST-STAGE GAGE NO 4 NR DEER PA 02232155 PENNYWASH CREEK NR DEER PARK

PENNYWASH CREEK AT MOUTH
SW $\quad 616$

SW 661

SW $\quad 674$

SW $\quad 31.7$

SW $\quad 23.3$

SW $\quad 4.36$

SW $\quad 30.2$

SW 248

SW 260

SW 953

SW 968

SW 1,046

SW 1,075

SW $\quad 17.2$

SW 22.1
27/44/25 80/46/30 FORT DRUM NE

$27 / 49 / 1980 / 48 / 43$ KENANSVILLE. SE 27/54/42 80/46/13 KENANSVILLE NE $27 / 59 / 4380 / 47 / 30$ KENANSVILLE NE 28/00/47 80/46/13 DEER PARK SE 28/01/53 81/02/21 HOLOPAW SE 27/56/38 80/59/50 KENANSVILLE $27 / 57 / 5981 / 00 / 23$ LAKE MARIAN NE 28/07/48 81/00/07 HOLOPAW 28/04/27 80/53/18 DEER PARK 28/02/44 80/48/56 OEER PARK SE 28/03/27 80/46/51 DEER PARK SE 28/05/03 80/45/11 DEER PARK SE 28/11/45 80/47/05 DEER PARK NE 28/11/45 80/51/25 DEER PARK NE 28/10/54 80/53/44 DEER PARK NW $28 / 11 / 2980 / 52 / 18$ DEER PARK NE
061

009

009

009

097

097

097

097

009

009

009

009

097 
DRAINAGE AREAS FOR SELECTEO STREAM SITES IN FLORIDA

ST. JOHNS RIVERT. JOHNS RIVER

SITE

NUMBER

NAME AND LOCATION

02232160 ST JOHNS RIVER CREST STAGE GAGE NO 5 NR DEER PA 02232180 ST JOHNS RIVER CREST-STAGE GAGE NO 6 NR DEER PA 02232200 WOLF CREEK NR DEER PARK

WOLF CREEK AT MOUTH

02232240 ST JOHNS RIVER CSG NO 6A AT LK WINDER NR COCOA 02232260 ST JOHNS RIVER CREST STAGE GAGE NO 7 NR COCOA 02232350 ST JOHNS RIVER CREST STAGE GAGE NO 7A NR COCOA 02232400 ST JOHNS RIVER NR COCOA 02232410 ST JOHNS RIVER CREST STAGE GAGE NO $7 \mathrm{~B}$ NR COCOA 02232413 TAYLOR CREEK AT S-164 NR COCOA 02232415 TAYLOR CREEK NR COCOA

02232430 ST JOHNS RIVER CREST STAGE GAGE NO 8 NR CHRISTM 02232450 JIM CREEK NEAR CHRISTMAS

02232451 JIM CREEK AT COUNTY ROAD NR CHRISTMAS

02232455 SECOND CREEK NR CHRISTMAS

02232458 SETTLEMENT CREEK NR CHRISTMAS

02232500 ST JOHNS RIVER NR CHRISTMAS

02232550 TOOTOOSAHATCHEE CREEK NR CHRISTMAS

02232660 SIXMILE CREEK NR MIMS

02232699 ST JOHNS RIVER NR TITUSVILLE

02232700 ST JOHNS RIVER CREST STAGE GAGE NO 9 NR CHRISTM 02232702 CHRISTMAS CREEK NR CHRISTMAS

02232705 JOSHUA CREEK NR CHRISTMAS

02232712 ROBERTS BRANCH NR BITHLO

02233000 OISSTON CANAL NR BITHLO

02233001 ECONLOCKHATCHEE RIVER AT MAGNOLIA RANCH NR BITH

DRAINAGE DRAINAGE

SITE AREA ARE LONG LAT CNTY

1,100

SW 1,101

SW $\quad 25.7$

SW 31.2

SW 1,180

SW 1,209

SW 1,273

SW 1,331

SW 1,339

SW

SW

SW

SW

SW

SW

SW

SW

SW

SW

SW

SW

SW

SW

SW

$\mathrm{CN}$

Sw
28/12/35 80/51/25 DEER PARK NE 28/13/10 80/52/00 DEER PARK NE 28/12/46 80/54/40 DEER PARK NW 28/13/21 80/52/28 DEER PARK NE 28/16/02 80/51/28 LAKE POINSETT 009 $28 / 17 / 2080 / 49 / 12$ LAKE POINSETT 009 28/22/06 80/52/20 LAKE POINSETT 009 28/22/10 80/52/22 LAKE POINSETT 009 28/22/12 80/52/22 LAKE POINSETT 009 28/20/23 80/56/06 LAKE POINSETT SW 097 28/21/08 80/55/43 LAKE POINSETT SW 009 28/29/21 80/52/55 LAKE POINSETT NW 095 28/26/07 80/57/58 LAKE POINSETT NW 095 28/26/16 80/57/44 LAKE POINSETT NW 095 28/26/54 80/58/25 LAKE POINSETT NW 095 28/27/33 80/59/19 LAKE POINSETT NW 095 28/32/34 80/56/40 TITUSVILLE SW 095 28/30/04 81/00/03 BITHLO 095 28/39/55 80/54/27 AURANTIA 009 $28 / 36 / 1380 / 58 / 13$ TITUSVILLE SW 009 28/36/36 80/57/54 TITUSVILLE SW 009 28/34/08 81/01/36 BITHLO 095 28/34/31 81/03/11 BITHLO 095 28/35/54 81/04/32 BITHLO 095 28/25/20 81/07/17 NARCOOSEE NE 095 28/25/27 81/07/10 NARCOOSEE NE 095 
DRAINAGE AREAS FOR SELECTED STREAM SITES IN FIORIDA

ST. JOHNS RIVER BASIN JOHNS RIVFR

SITE

NUMBER

NAME AND LOCATION

02233100 ECONLOCKHATCHEE RIVER NR BITHLO

02233102 ECONLOCKHATCHEE RIVER TRIBUTARY NR BITHLO

02233104 ECONLOCKHATCHEE RIVER AT STATE HIGHWAY 420 NR B

02233200 LITTLE ECONLOCKHATCHEE RIVER NR UNION PARK

02233300 LITTLE ECONLOCKHATCHEE RIVER AT UNION PARK

02233446 SUSANNA - CORRINE DITCH AT ORLANOO

02233464 LITTLE ECONLOCKHATCHEE RIVER NR OVIEDO

02233474 LITTLE ECONLOCKHATCHEE R AT IRON BR NR OVIEDO

LITTLE ECONLOCKHATCHEE RIVER AT MOUTH

02233484 ECONLOCKHATCHEE RIVER NR OVIEDO

02233750 MILLS BRANCH AT CHULUOTA

ECONLOCKHATCHEE RIVER AT MOUTH

02234000 ST JOHNS RIVER ABOVE LAKE HARNEY NR GENEVA

02234010 ST JOHNS RIVER AT OSCEOLA

02234150 COW CREEK NR MAYTOWN

COW CREEK AT MOUTH

02234207 ADAIR-CONCORD CANAL AT ORLANDO

02234216 CONCORD-IVANHOE CULVERT AT ORLANDO

02234262 LAKE SUE OUTLET AT ORLANDO

02234302 HOWELL CREEK NR MAITLAND

02234308 HOWELL CREEK NR ALTAMONTE SPRINGS

02234322 HOWELL CREEK NR OVIEDO

02234324 HOWELL CREEK NR SLAVIA

02234344 HOWELL CREEK AT STATE HIGHWAY 419 NEAR OVIEDO

$\begin{array}{lcllll} & \text { DRAINAGE } & \text { DRAINAGE } & & \\ \text { SITE } & \text { AREA } & \text { AREA } & \text { LAT } & \text { LONG } & \\ \text { TYPE } & \text { SQ.MI. } & \text { QUALIFIER DG/MI/SD DG/MI/SD } & \text { QUADRANGLE NAME } & \text { CODE }\end{array}$

QUALIFIER DG/MI/SD DG/MI/SD QUADRANGLE NAME CODE

SW $\quad 1.83$

SW 129

SW $\quad 27.1$

SW $\quad 39.4$

$\mathrm{CN} \quad .63$

SW $\quad 59.9$

SW $\quad 70.0$

SW 81.7

SW $\quad 225$

SW 241

SW $\quad 8.75$

SW 267

SW 2,043

SW 2.092

SW $\quad 14.8$

SW $\quad 17.6$

$\begin{array}{ll}C N & 1.27\end{array}$

DR $\quad 2.10$

OT $\quad 6.34$

SW $\quad 20.6$

SW 22.0

SW $\quad 28.9$

SW 29.2

SW $\quad 52.0$
28/33/54 81/09/16 OVIEDO SW 095

28/33/55 81/11/19 OVIEDO SW 095

28/31/29 $81 / 14 / 39$ OVIEDO SW 095

28/34/06 81/15/06 ORLANDO EAST 095

28/33/57 81/19/16 ORLANDO EAST 095

28/34/23 81/15/11 ORLANDO EAST 117

$28 / 36 / 3781 / 13 / 33$ OVIEDO SW 117

$28 / 39 / 1581 / 10 / 14$ OVIEDO

$28 / 39 / 1981 / 10 / 12$ OVIEDO 117

28/40/40 81/06/51 GENEVA 117

28/37/53 81/07/21 GENEVA 117

$28 / 42 / 1381 / 01 / 42$ GENEVA

28/42/50 81/02/08 GENEVA

117

$28 / 47 / 3781 / 03 / 29$ OSCEOLA

117

28/50/13 81/01/32 OSCEOLA

127

$28 / 48 / 3081 / 03 / 10$ OSCEOLA

$28 / 33 / 2881 / 23 / 19$ ORLANDO WEST

095

$28 / 33 / 3081 / 22 / 56$ ORLANDO WEST

$28 / 34 / 5281 / 21 / 24$ ORLANDO EAST

095

095

$28 / 37 / 2781 / 20 / 33$ ORLANDO EAST 095

28/37/54 81/19/28 CASSELBERRY 117

28/38/35 81/16/00 CASSELBERRY 117

28/38/51 81/15/53 CASSELBERRY 117

$28 / 41 / 2381 / 14 / 52$ OVIEDO
28/34/59 81/10/07 OVIEDO SW 095 
DRAINAGE AREAS FOR SELECTED STREAM SITES IN FLORIDA
ST. JOHNS RIVER

ST. JOHNS RIVER BASIN AGOVE OKLAWAHA RIVER

SITE

NUMBER

NAME ANO LOCATION

HOWELL CREEK AT MOUTH

02234384 SOLDIER CREEK NR LONGWOOD

02234400 GEE CREEK NR LONGWOOD

GEE CREEK AT MOUTH

SOLDIER CREEK AT MOUTH

02234435 ST JOHNS RIVER AT ST HWY 46 NR SANFORD

02234440 ST JOHNS RIVER ABOVE LAKE MONROE NR SANFORD

02234500 ST JOHNS RIVER NR SANFORD

02234635 WEKIVA RIVER NR APOPKA

02234815 LAKE WEKIVA OUTLET NR MAITLAND

02234945 LITTLE WEKIVA RIVER AT FOREST CITY
DRAINAGE DRAINAGE

AREA AREA

AREA LAT LONG CNTY

SW $\quad 53.0 \quad 28 / 42 / 0381 / 15 / 08$ CASSELBERRY

SW 21.2 28/43/07 81/18/32 CASSELBERRY

SW $12.8 \quad 28 / 42 / 13 \quad 81 / 17 / 27$ CASSELBERRY

SW $\quad 13.5 \quad 28 / 42 / 55 \quad 81 / 17 / 25$ CASSELBERRY

SW $\quad 36.8 \quad 28 / 42 / 58 \quad 81 / 17 / 23$ CASSELBERRY

SW 2,445

SW $\quad 2,456$

SW 2,582

SW $\quad 58.3$

CN $\quad 13.4$

SW $\quad 73.8$

02234947 LITTLE WEKIVA RIVER AT STATE HIGHWAY 436 AT FOR SW 73.9

02234988 CRANES ROOST AT ALTAMONTE SPRINGS

02234990 LITTLE WEKIVA RIVER NR ALTAMONTE SPRINGS

LITTLE WEKIVA RIVER AT MOUTH

OT $\quad 2.89$

SW $\quad 90.7$

SW $\quad 102$

02235000 WEKIVA RIVER NR SANFORD

02235155 BLACK WATER CREEK NR ALTOONA

02235200 BLACK WATER CREEK NR CASSIA

BLACK WATER CREEK AT MOUTH

WEKIVA RIVER AT MOUTH

02236000 ST JOHNS RIVER NR DELAND

02236010 ST JOHNS RIVER AT ST FRANCIS LANDING NR DE. LAND

02236120 DEEP CREEK NR BARBERVILLE

02236125 ST JOHNS RIVER AT ASTOR

02236157 PRICE CREEK NR PIERSON

ST JOHNS RIVER ABOVE OKLAWAHA RIVER
SW $\quad 189$

SW $\quad 32.5$

SW $\quad 126$

SW $\quad 196$

SW $\quad 396$

SW 3,066

SW $\quad 3,072$

SW $\quad 35.4$

SW $\quad 3,330$

SW 6.21 NONCONTR

SW $\quad 3,753$
117

117

117

28/47/09 $81 / 10 / 50$ OSTEEN

117

117

28/42/48 81/26/44 FOREST CITY 095

28/36/10 81/25/38 ORLANDO WEST 095

28/39/42 81/24/37 FOREST CITY 117

28/39/57 81/23/18 FOREST CITY 117

28/41/13 81/23/50 FOREST CITY 117

$28 / 45 / 2081 / 24 / 59$ SANFORD SW

$28 / 48 / 5481 / 25 / 10$ SANFORD SW

28/57/37 81/34/48 PAISLEY

069

28/52/37 81/29/21 PINE LAKES

069

28/52/05 81/22/53 SANFORD SW

28/52/36 81/22/01 ORANGE CITY

29/00/29 81/22/58 LAKE WOODRUFF

069

29/02/14 81/25/05 LAKE WOODRUFF

069

29/09/47 81/23/27 PIERSON

127

29/10/00 81/31/20 ASTOR

069

127
28/48/08 81/12/41 OSTEEN

28/39/46 81/24/38 FOREST CITY 117 
SITE

NUMBER

NAME AND LOCATION

02236300 GREEN SWAMP RUN NR LOUGHMAN

02236500 BIG CREEK NR CLERMONT

02236504 BEAR BRANCH NR CLERMONT

BIG CREEK AT MOUTH

02236600 LITTLE CREEK AT COOPERS RANCH NR CLERMONT

02236700 LITTLE CREEK NR CLERMONT

02236800 LAKE GLONA OUTLET NR CLERMONT

02236900 PALATLAKAHA RIVER AT CHERRY LAKE OUTLET NR GROV

02236901 PALATLAKAHA RIVER BL SPILLWAY AT CH LK O NR GRO

02236905 PALATLAKAHA RIVER AT GROVELAND

02237001 PALATLAKAHA RIVER BL SPWY NR MASCOTTE

02237205 PALATLAKAHA RIVER NR OKAHUMPKA

02237293 PALATLAKAHA RIVER ABOVE STR M-1 NR OKAHUMPKA

02237339 HELENA RUN NR OKAHUMPKA

HELENA RUN AT CONFLUENCE WITH PALATLAKAHA RIVER

PALATLAKAHA RIVER AT MOUTH

02237522 DEAD RIVER NR TAVARES

02237541 JOHNS LAKE OUTLET AT OAKLAND

02237700 APOPKA BEAUCLAIR CANAL NR ASTATULA

02237701 APOPKA BEAUCLAIR CANAL BELOW DAM NR ASTATULA

02237710 APOPKA-BEAUCLAIR CA AT STATE HWY 448 NR ASTATUL

02237732 WOLF BRANCH NR MT DORA

02237802 DORA CANAL AT TAVARES

02238000 HAINES CREEK AT LISBON

\begin{tabular}{|c|c|c|c|c|c|c|}
\hline $\begin{array}{l}\text { SITE } \\
\text { TYPE }\end{array}$ & $\begin{array}{l}\text { DRAINAGE } \\
\text { AREA } \\
\text { SQ.MI. }\end{array}$ & $\begin{array}{l}\text { DRAINAGE } \\
\text { AREA } \\
\text { QUALIF IER }\end{array}$ & $\begin{array}{l}\text { LAT } \\
D G / M I / S D\end{array}$ & $\begin{array}{c}\text { LONG } \\
\mathrm{DG} / \mathrm{MI} / \mathrm{SD}\end{array}$ & QUADRANGLE NAME & $\begin{array}{l}\text { CNTY } \\
\text { CODE }\end{array}$ \\
\hline OT & 33 & APPROX & $28 / 15 / 19$ & $81 / 40 / 32$ & LAKE LOUISA SW & 105 \\
\hline SW & 68 & APPROX & $28 / 26 / 51$ & $81 / 44 / 25$ & LAMONT SE & 069 \\
\hline OT & 1.83 & & $28 / 27 / 00$ & $81 / 44 / 25$ & LAKE LOUISA & .069 \\
\hline SW & 70 & APPROX & $28 / 27 / 29$ & $81 / 44 / 10$ & LAKE LOUISA & \\
\hline SW & 9.9 & APPROX & $28 / 25 / 50$ & $81 / 47 / 43$ & LAKE NELLIE & 069 \\
\hline SW & 14.70 & & $28 / 27 / 39$ & $81 / 45 / 26$ & LAKE NELLIE & 069 \\
\hline OT & 8.4 & APPROX & $28 / 28 / 43$ & $81 / 47 / 10$ & LAKE NELLIE & 069 \\
\hline SW & 165 & & $28 / 35 / 33$ & $81 / 49 / 21$ & CLERMONT WEST & 069 \\
\hline SW & 165 & & $28 / 35 / 32$ & $81 / 49 / 22$ & CLERMONT WEST & 069 \\
\hline SW & 177 & & $28 / 34 / 37$ & $81 / 51 / 21$ & CLERMONT WEST & 069 \\
\hline SW & 182 & & $28 / 36 / 56$ & $81 / 51 / 53$ & CLERMONT WEST & 069 \\
\hline SW & 182 & & $28 / 36 / 57$ & $81 / 51 / 58$ & CLERMONT WEST & 069 \\
\hline SW & 208 & & $28 / 43 / 00$ & $81 / 53 / 00$ & CENTER HILL & 069 \\
\hline SW & 221 & & $28 / 44 / 39$ & $81 / 52 / 22$ & HOWEY IN THE HILL & 069 \\
\hline SW & 15.6 & & $28 / 45 / 52$ & $81 / 52 / .53$ & LEESBURG WEST & 069 \\
\hline SW & 15.7 & & $28 / 45 / 51$ & $81 / 52 / 43$ & LEESBURG WEST & \\
\hline SW & 238 & & $28 / 46 / 06$ & $81 / 52 / 41$ & LEESBURG WEST & \\
\hline SW & 357 & & $28 / 48 / 41$ & $81 / 46 / 16$ & LEESBURG EAST & 069 \\
\hline $\mathrm{DR}$ & 40.5 & & $28 / 30 / 02$ & $81 / 37 / 17$ & WINTER GARDEN & 095 \\
\hline $\mathrm{CN}$ & 184 & & $28 / 43 / 20$ & $81 / 41 / 06$ & ASTATULA & 069 \\
\hline $\mathrm{CN}$ & 184 & & $28 / 43 / 22$ & $81 / 41 / 06$ & ASTATULA & 069 \\
\hline $\mathrm{CN}$ & 188 & & $28 / 44 / 48$ & $81 / 41 / 21$ & ASTATULA & 069 \\
\hline SW & 2.32 & & $28 / 48 / 54$ & $81 / 36 / 57$ & SORRENTO & 069 \\
\hline $\mathrm{CN}$ & 238 & & $28 / 48 / 39$ & $81 / 44 / 33$ & EUSTIS & 069 \\
\hline SW & 648 & & $28 / 52 / 20$ & $81 / 46 / 50$ & LEESBURG EAST & 069 \\
\hline
\end{tabular}


SITE
NUMBER

NAME AND LOCATION

02238001 HAINES CREEK BELOW BURRELL DAM AT LISBON

02238204 YALE GRIFFIN CANAL. NR LISBON

02238499 OKLAWAHA RIVER ABOVE. MOSS BLUFF DAM AT MOSS BLU

02238500 OKLAWAHA RIVER AT MOSS BLUFF

02238550 OKLAWAHA RIVER AT OKLAWAHA FARMS BRIDGE NR MOSS

02238801 LAKE WEIR OUTLET AT OKLAWAHA

02239000 OKLAWAHA RIVER NR OCALA

02240000 OKLAWAHA RIVER NR CONNER

02240440 EATON CREEK NR EUREKA

EATON CREEK AT MOUTH

02240500 OKLAWAHA RIVER AT EUREKA

02240800 HATCHET CREEK NR GAINESVILLE

HATCHET CREEK AT MOUTH

02240810 LITTLE HATCHET CREEK NR GAINESVILLE

02240813 NEWNANS LAKE TRIBUTARY NR GAINESVILLE

02240817 NEWNANS LAKE TRIBUTARY NO 2 NR GAINESVILLE

02240902 PRAIRIE CREEK NR GAINESVILLE

02240920 FAIRFIELD SINK DRAIN AT FAIRFIELD

02240940 LEVY LAKE CREEK NR MICANOPY

LEVY LAKE CREEK AT MOUTH

02240950 HOGTOWN CREEK NR GAINESVILLE

02240954 HOGTOWN CREEK NR ARREDONDO

02240960 PAYNES PRAIRIE NR MICANOPY

02240984 SWEETWATER BRANCH TRIBUTARY AT GAINESVILLE

02241000 CAMPS CANAL NR ROCHELLE

DRAINAGE DRAINAGE

AREA LONG CNTY $\begin{array}{llll}\text { SITE } & \text { AREA } & \text { AREA } & \text { LAT } \\ \text { TYPE } & \text { SQ.MI. QUALIFIER DG/MI/SD DG/MI/SD QUADRANGLE NAME } & \text { CNTY } \\ & & \text { CODE }\end{array}$

SW $648 \quad 28 / 52 / 20 \quad 81 / 46 / 50$ LEESBURG EAST 069

$\mathrm{CN} \quad 68.1$

SW $\quad 879$

SW $\quad 879$

SW $\quad 886$

DR $\quad 53.8$

SW 1,018

SW 1,196

SW $\quad 84.6$

SW $\quad 108$

SW 1,367

SW

65.2

65.4

10.2

.74

2.24

114

1.70

.47

1.66

18.5

41.2

650 APPROX

.79

775
28/55/17 81/46/26 EMERALDA ISLAND 069

29/04/52 81/52/51 LAKE WEIR 083

29/04/55 81/53/05 LAKE WEIR 083

29/05/58 81/54/42 LAKE WEIR 083

29/02/40 81/55/30 LAKE WEIR 083

29/11/00 81/59/40 LYNNE 083

29/12/50 81/59/10 LYNNE 083

29/20/15 81/53/00 FORT MCCOY 083

29/20/14 81/52/50 FORT MCCOY 083

29/22/20 81/54/10 FORT MCCOY 083

$29 / 41 / 1582 / 12 / 24$ ORANGE HEIGHTS 001

29/41/00 82/12/00 ORANGE HEIGHTS

29/40/56 82/13/51 ORANGE HEIGHTS 001

29/40/45 82/14/03 ORANGE HEIGHTS 001

$29 / 39 / 4482 / 15 / 14$ GAINESVILLE EAST 001

29/36/3982/14/54 ROCHELLE 001

29/21/58 82/15/17 FAIRFIELD 083

29/30/59 82/17/42 MICANOPY 001

0/00/00 0/00/00 MICANOPY 001

29/39/01 82/22/32 GAINESVILLE WEST 001

$29 / 38 / 1782 / 23 / 33$ GAINESVILLE WEST 001

29/33/50 82/20/00 MICANOPY 001

29/39/00 82/19/14 GAINESVILLE EAST 001

$29 / 34 / 3,482 / 14 / 59$ ROCHELLE

001 
DRAINAGE AREAS FOR SELECTED STREAM SITES IN FLORIDA

ST. JOHNS RIVER

OKLAWAHA RIVER BASIN

SITE
NUMBBER

NAME AND LOCATION

02241100 RIVER STYX NR MICANOPY

02241800 LOCHLOOSA CREEK NR MELROSE

02241900 LOCHLOOSA CREEK AT GROVE PARK

02242000 LOCHLOOSA CREEK NR HAWTHORNE

LOCHLOOSA CREEK AT MOUTH

02242005 LOCHLOOSA LAKE TRIBUTARY AT HAWTHORNE

02242007 TRIBUTARY TO LOCHLOOSA LAKE TRIBUTARY NR HAWTHO

02242451 ORANGE. LAKE OUTLET NR CITRA

02243000 ORANGE CREEK AT ORANGE SPRINGS

02243400 LITTLE ORANGE CREEK NR ORANGE SPRINGS

LITTLE ORANGE CREEK AT MOUTH

ORANGE CREEK AT MOUTH

02243500 OKLAWAHA RIVER NR ORANGE SPRINGS

02243530 BRUNTBRIDGE BROOK AT KENWOOD

02243609 DEEP CREEK NR KENWOOD

02243682 SWEETWATER CREEK NR HOLLISTER

02243800 DEEP CREEK NR RODMAN

02243958 LAKE OCKLAWAHA NR ORANGE SPRINGS

02243959 OKLAWAHA RIVER ABOVE RODMAN DAM NR ORANGE SPRIN

02243960 OKLAWAHA RIVER AT RODMAN DAM NR ORANGE SPRINGS

02244000 OKLAWAHA R AT RIVERSIDE LANDING NR ORANGE SPRIN

02244005 OKLAWAHA RIVER AT ST HWY 19 NR SALT SPRINGS

OKLAWAHA RIVER AT MOUTH

02244040 ST JOHNS RIVER AT BUFFALO BLUFF NR PALATKA

\begin{tabular}{|c|c|c|c|c|c|c|}
\hline $\begin{array}{l}\text { SITE } \\
\text { TYPE }\end{array}$ & $\begin{array}{c}\text { DRAINAGE } \\
\text { AREA } \\
\text { SQ.MI. }\end{array}$ & $\begin{array}{l}\text { DRAINAGE } \\
\text { AREA } \\
\text { QUALIF IER }\end{array}$ & $\begin{array}{c}\text { LAT } \\
\mathrm{DG} / \mathrm{MI} / \mathrm{SD}\end{array}$ & $\begin{array}{c}\text { LONG } \\
\mathrm{DG} / \mathrm{MI} / \mathrm{SD}\end{array}$ & QUADRANGLE NAME & $\begin{array}{l}\text { CNTY } \\
\text { CODE }\end{array}$ \\
\hline SW & 800. & & $29 / 31 / 00$ & $82 / 13 / 22$ & ROCHELLE & 001 \\
\hline SW & 2.79 & & $29 / 42 / 07$ & $82 / 08 / 02$ & ORANGE HEIGHTS & 001 \\
\hline SW & 37.4 & & $29 / 36 / 00$ & $82 / 08 / 42$ & ROCHELLE & 001 \\
\hline SW & 46.3 & & $29 / 33 / 53$ & $82 / 08 / 25$ & ROCHELLE & 001 \\
\hline SW & 46.9 & & $29 / 35 / 00$ & $82 / 09 / 00$ & ROCHELLE & 001 \\
\hline SW & 1.18 & & $29 / 35 / 24$ & $82 / 06 / 07$ & HAWTHORNE & 001 \\
\hline SW & .12 & & $29 / 35 / 24$ & $82 / 06 / 18$ & HAWTHORNE & 001 \\
\hline OT & 1,012 & & $29 / 26 / 30$ & $82 / 06 / 35$ & CITRA & 001 \\
\hline SW & 1,119 & & $29 / 30 / 34$ & $81 / 56 / 47$ & KEUKA & 083 \\
\hline SW & 75.5 & & $29 / 31 / 22$ & $81 / 56 / 23$ & KEUKA & 107 \\
\hline SW. & 76.2 & & $29 / 30 / 44$ & $81 / 56 / 28$ & KEUKA & \\
\hline SW & 1,198 & & & & KEUKA & \\
\hline SW & 2,657 & & $29 / 30 / 13$ & $81 / 54 / 43$ & KEUKA & 083 \\
\hline SW & 4.63 & & $29 / 32 / 08$ & $81 / 53 / 02$ & KEUKA & 107 \\
\hline SW & 6.34 & & $29 / 34 / 12$ & $81 / 52 / 41$ & KEUKA & 107 \\
\hline SW & 4.90 & & $29 / 37 / 43$ & $81 / 49 / 46$ & BAYWOOD & 107 \\
\hline SW & 54.3 & & $29 / 32 / 28$ & $81 / 50 / 12$ & RODMAN & 107 \\
\hline RE & 2,747 & & $29 / 30 / 30$ & $81 / 48 / 15$ & RODMAN & 107 \\
\hline SW & 2,747 & & $29 / 30 / 30$ & $81 / 48 / 15$ & RODMAN & 107 \\
\hline SW & 2,747 & & $29 / 30 / 30$ & $81 / 48 / 15$ & RODMAN & 107 \\
\hline SW & 2,747 & & $29 / 30 / 00$ & $81 / 48 / 00$ & LK DELANCY & 107 \\
\hline SW & 2,762 & & $29 / 29 / 00$ & $81 / 44 / 00$ & WELAKA & 107 \\
\hline SW & 2,769 & APPROX & $29 / 28 / 15$ & $81 / 41 / 15$ & WELAKA & \\
\hline SW & 6.582 & & $29 / 35 / 46$ & $81 / 41 / 00$ & SATSUMA & 107 \\
\hline
\end{tabular}


DRAINAGE AREAS FOR SELECTEO STREAM SITES IN FLORIDA

ST. JOHNS RIVER BASIN BELOW OKLAWAHA RIVER

SITE

NAME AND LOCATION

02244100 SWEETWATER BRANCH NR BUNNELL

02244150 BLACK BRANCH NR BUNNELL

02244200 HAW CREEK NR BUNNELL

02244300 MIDDLE HAW CREEK AT RELAY STATION NR BUNNELL

02244320 MIDDLE HAW CREEK NR KORONA

02244330 MIDDLE HAW CREEK NR BUNNELL

02244400 LITTLE HAW CREEK AT STATE HIGHWAY 11 NR BUNNELL

02244420 LITTLE HAW CREEK NR SEVILLE

HAW CREEK AT MOUTH

02244440 DUNNS CREEK NR SATSUMA

DUNNS CREEK AT MOUTH

02244450 ST. JOHNS RIVER AT PALATKA

02244470 PALMETTO BRANCH TRIBUTARY AT CARRAWAY

02244473 RICE CREEK NR SPRINGSIDE

02244477 RICE CREEK NR WOODBURN

02244551 BLUE POND OUTLET NR KEYSTONE HEIGHTS

02244651 MAGNOLIA LAKE OUTLET NR KEYSTONE HEIGHTS

02244751 BROOKLYN LAKE OUTLET AT KEYSTONE HEIGHTS

02244801 LAKE GENEVA OUTLET NR KEYSTONE HEIGHTS

02245000 ETONIA CREEK NR FLORAHOME

02245050 ETONIA CREEK AT BARDIN

02245053 ETONIA CREEK NR PALATKA

02245100 SIMMS CREEK NR W TOCOI

02245140 SIMMS CREEK NR BARDIN

02245150 SIMMS CREEK NR PALATKA

\begin{tabular}{cc} 
SITE & $\begin{array}{c}\text { DRAINAGE } \\
\text { AREA }\end{array}$ \\
TYPE & SQ.MI \\
SW & 11.1 \\
SW & 14.5 \\
SW & 109 \\
SW & 54.6 \\
SW & 78.3 \\
SW & 86.6 \\
SW & 66.4 \\
SW & 93.0 \\
SW & 230 \\
SW & 492 \\
SW & 498 \\
SW & 7,094 \\
SW & 2.94 \\
SW & 43.2 \\
SW & 51.1 \\
DR & 2.32 \\
DR & 14.8 \\
DR & 17.4 \\
DR & 35.5 \\
SW & 167 \\
SW & 219 \\
SW & 219 \\
SW & 4.33 \\
SW & 47.3 \\
\hline & 50.8
\end{tabular}

DRAINAGE

AREA LONG CNTY

QUALIFIER DG/MI/SD DG/MI/SD QUADRANGLE NAME CODE

$29 / 25 / 1081 / 18 / 00$ BUNNELL

035

$29 / 26 / 5081 / 16 / 30$ BUNNELL

035

$29 / 24 / 1081 / 21 / 10$ BUNNELL

035

29/18/22 81/16/10 CODYS CORNER 035

29/21/35 81/18/42 CODYS CORNER 035

29/22/10 81/21/10 BUNNELL 035

29/16/07 81/20/11 CODYS CORNER 035

29/19/20 81/23/10 SEVILLE

035

29/23/55 81/25/56 ST JOHNS PARK

29/34/39 81/37/35 SATSUMA

035

$29 / 35 / 2081 / 37 / 51$ SATSUMA

$29 / 35 / 4681 / 36 / 29$ SAN MATEO 107

29/42/38 81/47/33 BAYWOOD 107

29/41/17 81/44/32 PALATKA 107

29/41/40 81/43/30 PALATKA 107

29/52/08 82/01/20 KEYSTONE HEIGHTS 019

29/49/02 82/01/21 KEYSTONE HEIGHTS 019

29/47/18 82/02/10 KEYSTONE HEIGHTS 019

29/45/15 82/00/35 KEYSTONE HEIGHTS 019

29/44/08 81/51/47 BAYWOOD 107

29/43/00 81/43/31 PALATKA 107

29/42/50 81/42/30 PALATKA 107

29/50/16 81/42/22 BOSTWICK 019

29/44/26 81/42/49 PALATKA 107

29/43/00 81/42/30 PALATKA 107 
DRAINAGE AREAS FOR SELECTED STREAM SITES IN FLORIDA

ST. JOHNS RIVER BASIN BELOW OKLAWAHA RIVER

SITE
NUMBER

NAME AND LOCATION

SIMMS CREEK AT MOUTH

ETONIA CREEK AT MOUTH

02245200 RICE CREEK NR PALATKA

RICE CREEK AT MOUTH

02245250 SIXTEENMILE CREEK NR HASTINGS

02245255 DEEP CREEK NR HASTINGS

02245265 CRACKER BRANCH AT HASTINGS

02245280 MOCCASIN BRANCH AT ARMSTRONG

02245285 MC CULLOUGH CREEK NR RIVERDALE

02245300 CLARKS CREEK NR GREEN COVE SPRINGS

02245315 SIXMILE CREEK AT BAKERSVILLE
02245325 MILL CREEK NR BAKERSVILLE

02245335 TROUT CREEK NR ORANGEDALE

02245350 GOVERNORS CREEK NR GREEN COVE SPRINGS

GOVERNORS CREEK AT MOUTH

02245400 SOUTH FORK BLACK CREEK NR CAMP BLANDING

02245430 ATES CREEK NR PENNY FARMS

02245470 GREENS CREEK NR PENNEY FARMS

02245500 SOUTH FORK BLACK CREEK NR PENNEY FARMS

02245600 BULL CREEK NR MIDDLEBURG

02245710 NORTH FORK BLACK CREEK NR KINGSLEY

02245750 NORTH FORK BLACK CREEK AB BOGGY BR NR HIGHLAND

02245760 BOGGY BRANCH NR HIGHLAND

02245800 NORTH FORK BLACK CREEK NR HIGHLAND

02245850 LONG BRANCH AT MAXVILLE

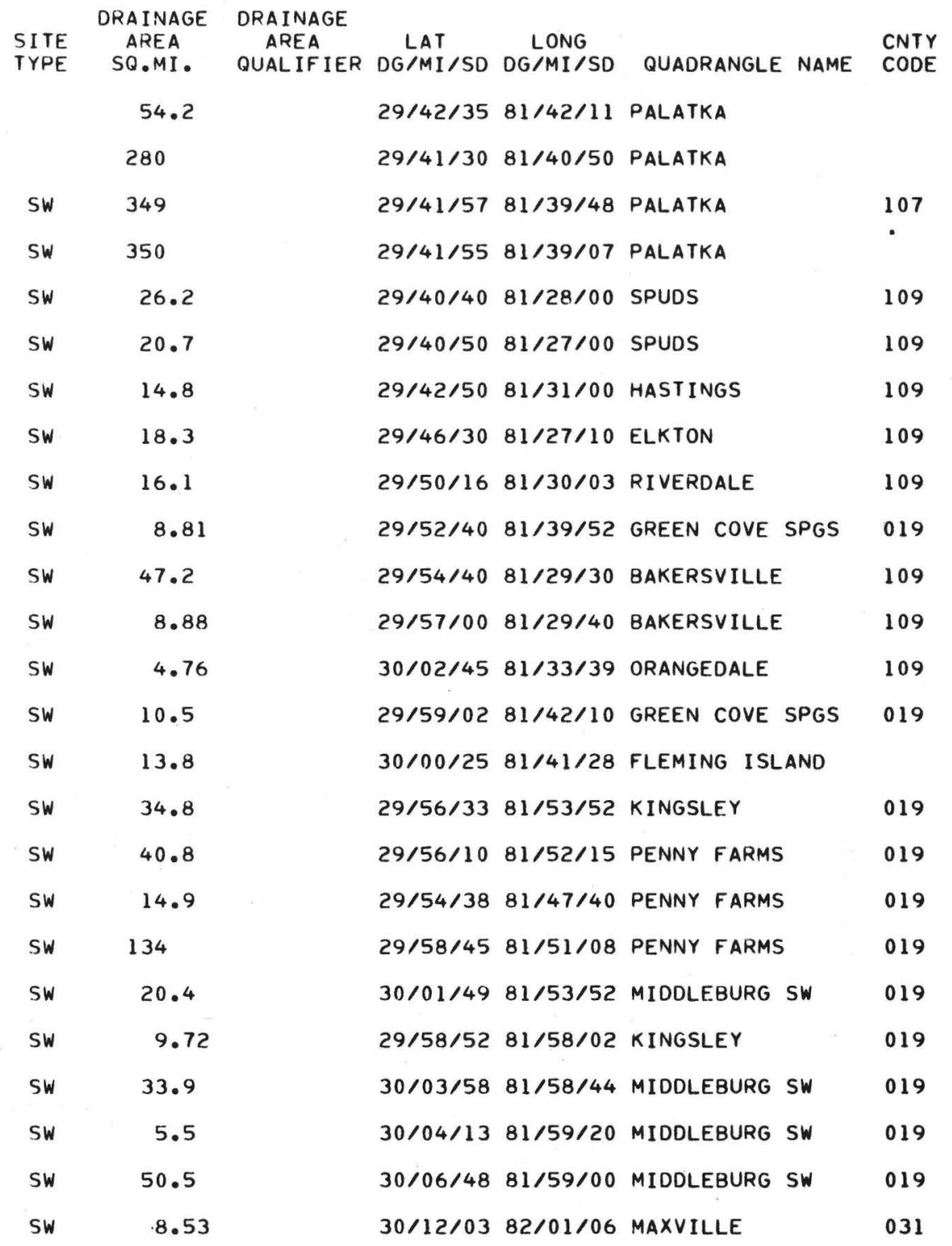


DRAINAGE AREAS FOR SELECTEO STREAM SITES IN FLORIDA

ST. JOHNS RIVER BASIN BELOW OKLAWAHA RIVER

SITE

NAME AND LOCATION

02245860 NORTH FORK BLACK CREEK NR MAXVILLE

02245900 YELLOW WATER CREEK NR MAXVILLE

02245920 SAL TAYLOR CREEK NR FIFTONE

02245930 YELLOW WATER CREEK NR HIGHLAND

02246000 NORTH FORK BLACK CREEK NR MIDDLEBURG

$$
\text { BLACK CREEK AT MOUTH }
$$

02246150 BIG DAVIS CREEK AT BAYARO

BIG DAVIS CREEK AT MOUTH

02246200 DURBIN CREEK NR DURBIN

DURBIN CREEK AT MOUTH

02246202 CORMORANT BRANCH NR MANDARIN

JULINGTON CREEK AT MOUTH

02246250 ST JOHNS RIVER AT USNAS NR JACKSONVILLE

02246300 ORTEGA RIVER AT JACKSONVILLE

02246450 WILLS BRANCH NR MARIETTA

02246455 SOUTH FORK WILLS BRANCH NR MARIETTA

02246460 WILLIAMSON CREEK AT CEDAR HILLS

02246497 MCCOY CREEK AT JACKSONVILLE

02246500 ST JOHNS RIVER AT JACKSONVILLE

02246515 POTTSBURG CREEK NR SOUTH JACKSONVILLE

02246522 RED BAY BRANCH TRIBUTARY AT JACKSONVILLE

02246530 ST JOHNS RIVER AT US DREDGE DEPOT AT JACKSONVIL

02246600 TROUT RIVER AT DINSMORE

02246602 LITTLE TROUT RIVER AT DINSMORE

02246645 SIXMILE CREEK AT PICKETTVILLE
DRAINAGE DRAINAGE

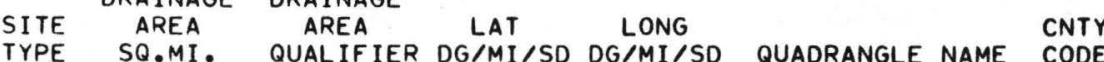

SW $\quad 101$

SW $\quad 21.9$

SW $\quad 27.5$

SW $\quad 56.5$

SW $\quad 177$

SW $\quad 488$

SW $\quad 13.6$

SW $\quad 15.6$

SW $\quad 36.7$

SW $\quad 44.4$

SW $\quad 1.62$

SW $\quad 100$

SW $\quad 8,618$

SW $\quad 30.9$

SW $\quad 7.04$

SW $\quad 2.00$

SW $\quad .92$

SW $\quad 3.51$

ES 8,754

SW $\quad 9.89$

CN $\quad .58$

8,800

20.9

SW $\quad 1.28$

SW $\quad 12.1$

$30 / 08 / 3381 / 55 / 48$ FIFTONE

031

$30 / 13 / 4481 / 55 / 17$ FIFTONE

031

$30 / 12 / 0081 / 54 / 41$ FIFTONE

031

$30 / 11 / 1281 / 55 / 13$ FIFTONE

019

30/06/47 81/54/24 MIDOLEBURG SW

30/02/31 81/42/41 FLEMING ISLAND

30/09/05 81/31/35 BAYARD

30/01/37 81/33/20 BAYARD

30/05/57 81/31/34 ORANGEDALE

$30 / 07 / 5081 / 36 / 01$ BAYARO

30/08/56 81/37/43 ORANGE PARK

30/07/37 81/38/19 ORANGE PARK

$30 / 13 / 3981 / 39 / 58$ ORANGE PARK

$30 / 14 / 5081 / 47 / 49$ JACKSONVILLE HEIG 031 30/17/29 81/46/00 MARIETTA

031

30/17/05 81/46/05 MARIETTA

031

30/16/19 81/45/05 MARIETTA

031

$30 / 19 / 3581 / 41 / 56$ JACKSONVILLE

031

30/19/16 81/39/54 JACKSONVILLE

031

$30 / 15 / 5081 / 35 / 25$ ARLINGTON

031

$30 / 20 / 4081 / 35 / 22$ ARLINGTON

031

$30 / 21 / 2581 / 37 / 18$ ARLINGTON

031

30/25/51 81/46/07 DINSMORE

031

30/26/23 81/46/21 DINSMORE

031

30/21/46 81/46/24 MARIETTA 
DRAINAGE AREAS FOR SELECTED STREAM SITES IN FLORIDA

ST. JOHNS RIVER BASIN BELOW OKLAWAHA RIVER

SITE
NUMBER

NAME ANO LOCATION

02246650 SIXMILE CREEK NR MARIETTA

02246700 LITTLE SIXMILE CREEK NR MARIETTA

02246709 RIBAULT RIVER NR JACKSONVILLE

TROUT RIVER AT MOUTH

02246750 CEDAR CREEK NR PANAMA PARK

02246754 PICKETT BRANCH NR FREEPORT

02246760 LITTLE CEDAR CREEK NR EASTPORT

02246800 DUNN CREEK NR EASTPORT

02246828 PABLO CREEK AT JACKSONVILLE

02246832 CEDAR SWAMP CREEK AT JACKSONVILLE

ST JOHNS RIVER AT MOUTH

\begin{tabular}{|c|c|c|c|c|c|c|}
\hline $\begin{array}{l}\text { SITE } \\
\text { TYPE }\end{array}$ & $\begin{array}{l}\text { DRAINAGE } \\
\text { AREA } \\
\text { SQ.MI. }\end{array}$ & $\begin{array}{l}\text { DRAINAGE } \\
\text { AREA } \\
\text { QUALIFIER }\end{array}$ & $\begin{array}{c}\text { LAT } \\
\mathrm{DG} / \mathrm{MI} / \mathrm{SD}\end{array}$ & $\begin{array}{c}\text { LONG } \\
\mathrm{DG} / \mathrm{MI} / \mathrm{SD}\end{array}$ & QUADRANGLE NAME & $\begin{array}{l}\text { CNTY } \\
\text { CODE }\end{array}$ \\
\hline SW & 16.1 & & $30 / 22 / 14$ & $81 / 44 / 47$ & JACKSONVILLE & 031 \\
\hline SW & 3.21 & & $30 / 21 / 51$ & $81 / 44 / 16$ & JACKSONVILLE & 031 \\
\hline SW & 20.7 & & $30 / 22 / 29$ & $81 / 43 / 37$ & JACKSONVILLE & 031 \\
\hline SW & 92.9 & & $30 / 23 / 25$ & $81 / 38 / 10$ & TROUT RIVER & \\
\hline SW & 6.27 & & $30 / 27 / 30$ & $81 / 40 / 49$ & TROUT RIVER & 031 \\
\hline SW & 3.01 & & $30 / 27 / 59$ & $81 / 40 / 05$ & TROUT RIVER & 031 \\
\hline SW & 2.96 & & $30 / 28 / 05$ & $81 / 39 / 23$ & TROUT RIVER & 031 \\
\hline SW & 4.86 & & $30 / 28 / 15$ & $81 / 36 / 07$ & EASTPORT & 031 \\
\hline SW & 25.8 & & $30 / 14 / 07$ & $81 / 28 / 42$ & PALM VALLEY & 031 \\
\hline SW & 3.40 & & $30 / 14 / 38$ & $81 / 28 / 26$ & PALM VALLEY & 031 \\
\hline SW & 9,168 & & $30 / 24 / 04$ & $81 / 24 / 08$ & MAYPORT & \\
\hline
\end{tabular}

COASTAL AREA BETWEEN ST. JOHNS RIVER AND PONCE DE LEGN INLET
SITE
NUMBER

NAME ANO LOCATION

02246900 MOULTRIE CREEK AT ST HWY 207 NR ST AUGUSTINE

02247000 MOULTRIE CREEK NR ST AUGUSTINE

02247010 FORT PEYTON BRANCH NR ST AUGUSTINE

MOULTRIE CREEK AT MOUTH

02247027 MOSES CREEK NR MOULTRIE

02247200 FISH SWAMP OUTLET NR SUMMER HAVEN

02247210 PELLICER CREEK NR SUMMER HAVEN

PELLICER CREEK AT MOUTH

02247480 TIGER BAY CANAL NR DAYTONA BEACH

\begin{tabular}{|c|c|c|c|c|c|c|}
\hline $\begin{array}{l}\text { SITE } \\
\text { TYPE }\end{array}$ & $\begin{array}{l}\text { DRAINAGE } \\
\text { AREA } \\
\text { SQ.MI. }\end{array}$ & $\begin{array}{l}\text { DRAINAGE } \\
\text { AREA } \\
\text { QUALIF IER }\end{array}$ & $\begin{array}{c}\text { LAT } \\
\mathrm{DG} / \mathrm{MI} / \mathrm{SD}\end{array}$ & $\begin{array}{c}\text { LONG } \\
\mathrm{DG} / \mathrm{MI} / \mathrm{SD}\end{array}$ & QUADRANGLE NAME & $\begin{array}{l}\text { CNTY } \\
\text { CODE }\end{array}$ \\
\hline SW & 19.80 & & $29 / 50 / 50$ & $81 / 21 / 39$ & ST AUGUSTINE BCH & 109 \\
\hline SW & 20.80 & & $29 / 49 / 40$ & $81 / 20 / 57$ & ST AUGUSTINE BCH & 109 \\
\hline OT & 7.62 & & $29 / 49 / 20$ & $81 / 20 / 50$ & ST AUGUSTINE BCH & 109 \\
\hline SW & 43.40 & & $29 / 49 / 48$ & $81 / 18 / 26$ & ST AUGUSTINE BCH & \\
\hline SW & 7.51 & & $29 / 46 / 28$ & $81 / 18 / 59$ & ST AUGUSTINE BCH & 109 \\
\hline SW & 4.62 & & $29 / 39 / 20$ & $81 / 19 / 43$ & DINNER ISLAND & 109 \\
\hline SW & 43.70 & & $29 / 39 / 02$ & $81 / 18 / 03$ & DINNER ISLAND & 109 \\
\hline SW & 84.00 & & $28 / 39 / 45$ & $81 / 14 / 06$ & MATANZAS INLET & \\
\hline $\mathrm{CN}$ & 29.00 & APPROX & $29 / 09 / 58$ & $81 / 09 / 18$ & DAYTONA BEACH NW & 127 \\
\hline
\end{tabular}


02247500 TOMOKA RIVER NR DAYTONA BEACH

02247510 TOMOKA RIVER NR HOLLY HILL

02247600 LITTLE TOMOKA RIVER NR ORMOND BEACH

LITTLE TOMOKA RIVER AT MOUTH

TOMOKA RIVER AT MOUTH

02248000 SPRUCE CREEK NR SAMSULA

02248010 SPRUCE CREEK TRIBUTARY NR SAMSULA

02248020 SPRUCE CREEK TRIBUTARY NO 2 NR SAMSULA

02248050 SPRUCE CREEK NR PORT ORANGE

SPRUCE CREEK AT MOUTH

$\begin{array}{cc}\text { SW } & 76.2 \\ \text { SW } & 76.8 \\ \text { SW } & 10.0 \\ \text { SW } & 20.0 \\ \text { SW } & 152 \\ \text { SW } & 33.4 \\ \text { SW } & 8.46 \\ \text { SW } & 9.87 \\ \text { SW } & 88.0 \\ \text { SW } & 111\end{array}$

29/12/35 81/06/20 DAYTONA BEACH

29/15/27 81/07/24 ORMOND BEACH 29/20/57 81/05/26 ORMOND BEACH

29/03/01 81/02/49 SAMSULA

COASTAL AREA BETWEEN PONCE DE LEON INLET AND SEBASTIAN INLET

SI TE
NUMBER

NAME AND LOCATION

SITE
TYPE $\begin{gathered}\text { DRAINAGE } \\ \text { AREA } \\ \text { SQ.MI. }\end{gathered}$

ORAINAGE

AREA LAT LONG

LAT
QUALA IFIER DG/MI/SD DG/MI/SD QUADRANGLE NAME

CNTY CODE

02249000 EAU GALLIE RIVER NR EAU GALLIE

02249500 CRANE CREEK AT MELBOURNE

CRANE CREEK AT MOUTH

02250000 TURKEY CREEK PALM BAY

02250500 GOAT CREEK NR VALKARIA
28/07/55 80/39/17 EAU GALLIE

28/04/42 80/37/48 MELBOURNE WEST

28/04/32 80/35/58 MELBOURNE EAST

28/00/46 80/37/20 MELBOURNE EAST

$27 / 58 / 0180 / 33 / 57$ GRANT
009

009

COASTAL AREA SEBASTIAÑ INLET TO ST. LUCIE RIVER

SITE

NAME AND LOCATION

02251500 NORTH PRONG SEBASTIAN CREEK NR MICCO

02251765 FELLSMERE CANAL NR FELLSMERE

02253600 MOORE CREEK AT FT PIERCE
DRÁINAGE DRAINAGE

SITE AREA AREA LAT LONG

LONG CNTY

SW 56.0 27/51/21 $80 / 31 / 28$ FELLSMERE

CN $78.4 \quad 27 / 49 / 1880 / 36 / 27$ FELLSMERE 061

SW $\quad 4.00 \quad 27 / 26 / 58 \quad 80 / 19 / 37$ FT. PIERCE 111 
SITE
NUMBER

NAME AND LOCATION

02261000 LIZZIE-COON CA NR ASHTON

02261500 MYRTLE-MARY JANE CANAL NR NARCOOSSEE

02262000 MARY JANE-HART CANAL NR NARCOOSSEE

02262500 AJAY-EAST TOHOPEKALIGA CANAL NR NARCOOSSEE

02262900 BOGGY CREEK NR TAFT

02263000 BOGGY CREEK NR KISSIMMEE

BOGGY CREEK AT MOUTH

02263500 ST CLOUD CANAL AT S-59 NR ST CLOUD

02263501 ST CLOUD CANAL BELOW S-59 NR ST CLOUD

02263700 SHINGLE CREEK NR VINELAND

02263800 SHINGLE CREEK AT AIRPORT NR KISSIMMEE

02263851 BAY LAKE OUTLET AT S105A NR VINELAND

02263869 SOUTH LAKE OUTLET AT S-15 NR VINELAND

02264000 CYPRESS CREEK AT VINELAND

02264100 BONNET CREEK NR VINELAND

02264500 SHINGLE CREEK NR KISSIMMEE

SHINGLE CREEK AT MOUTH

02265000 SOUTH PORT CANAL AT S-61 NR ST CLOUD

02265001 SOUTH PORT CANAL BELOW S-61 NR ST CLOUD

02266000 CANOE CREEK NR ST CLOUD

CANOE CREEK AT MOUTH

02266025 REEDY CREEK ABOVE S-46 NR VINELAND

02266200 WHITTENHORSE CREEK NR VINELAND

WHITTENHORSE CREEK AT MOUTH

02266291 LATERAL 405 AT S-405A NR DOCTOR PHILLIPS $\begin{array}{lcllll} & \text { DRAINAGE } & \text { DRAINAGE } & & \\ \text { SITE } & \text { AREA } & \text { AREA } & \text { LAT } & \text { LONG } & \\ \text { TYPE } & \text { SQ.MI• } & \text { QUALIFIER DG/MI/SD DG/MI/SD } & \text { QUADRANGLE NAME } & \text { CODE }\end{array}$

CN $\quad 31.5 \quad 28 / 15 / 2981 / 11 / 20$ LAKE POINSETT 097

CN $\quad 124$

CN 171

SW $\quad 83.6$

SW 86.3

SW $\quad 88.8$

$\mathrm{CN} \quad 308$

CN $\quad 308$

SW $\quad 48.0$

SW 89.2

CN $\quad 14.8$

CN $\quad 4.00$

SW $\quad 30.3$

SW $\quad 56.1$

SW $\quad 180$

SW $\quad 180$

APPROX

28/20/22 81/10/27 NARCOOSSEE

28/22/54 81/11/24 NARCOOSEE NW 095

$28 / 20 / 2381 / 13 / 42$ NARCOOSSEE

28/22/16 81/18/39 ST CLOUD NORTH

28/20/52 81/19/12 ST CLOUD NORTH

28/19/46 81/18/50 ST CLOUD NORTH

$28 / 15 / 5681 / 18 / 38$ ST CLOUD NORTH

28/15/56 81/18/38 ST CLOUD NORTH

28/24/36 81/26/02 LAKE JESSAMINE

$28 / 18 / 1481 / 27 / 04$ KISSIMMEE

28/24/48 81/33/27 WINDERMERE

28/24/45 81/32/17 WINDERMERE

$28 / 23 / 2581 / 31 / 11$ WINDERMERE

097

097

095

095

097

097

095

097

095

095

095

28/19/58 81/31/20 INTERCESSION CITY 097

28/16/01 81/26/53 KISSIMMEE

097

28/15/56 81/24/36 KISSIMEE

28/08/22 81/21/06 ST CLOUD SOUTH 097

28/08/22 81/21/06 ST CLOUD SOUTH 097

28/04/22 81/15/39 CYPRESS LAKE 097

28/04/28 81/17/50 CYPRESS LAKE

$28 / 24 / 1481 / 36 / 42$ WINDERMERE

095

28/23/05 81/37/00 WINDERMERE

095

28/23/38 81/36/28 WINDERMERE

$28 / 25 / 3481 / 36 / 23$ WINDERMERE

5

7

\section{Y}

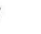

5

7


SITE
NUMBER

NAME AND LOCATION

02266300 REEDY CREEK NR VINELAND

02266550 REEOY CREEK NR POINIANNA

REEDY CREEK AT MOUTH

022667000 HORSE CREEK AT DAVENPORT

HORSE CREEK AT MOUTH

02267000 CATFISH CREEK NR LAKE WALES

CATFISH CREEK AT MOUTH

02268500 WEOHYAKAPKA CREEK NR LAKE WALES

02268903 KISSIMMEE RIVER AT S65 NR LAKE WALES

02.268904 KISSIMMEE RIVER BELOW S65 NR LAKE WALES $\begin{array}{cclccc} & \text { DRAINAGE } & \text { DRAINAGE } & & \\ \text { SITE } & \text { AREA } & \text { AREA } & \text { LAT } & \text { LONG } & \\ \text { TYPE } & \text { SQ.MI. } & \text { QUALIFIER DG/MI/SD DG/MI/SD } & \text { QUADRANGLE NAME } & \text { CODE }\end{array}$

SW 75.0 APPROX 28/19/57 81/34/48 INTERCESSION CITY 097

SW 170 APPROX 28/08/59 81/26/28 LAKE TOHOPEKALIGA 097

SW 190 APPROX 28/04/01 81/20/56 LAKE HATCHNEHA

SW 22.8 28/10/31 81/35/54 DAVENPORT 105

SW $\quad 26.6$

SW $\quad 58.9$

SW $\quad 76.3$

SW 96.8

SW 1,607

SW 1,607

SW $\quad 1,607$

02269040 KISSIMMEE RIVER CREST-STAGE GAGE NOI NR AVON PA SW 1,737

02269060 KISSIMMEE RIVER CREST-STAGE GAGE NO2 NR AVON PA SW 1,791

02269100 KISSIMMEE RIVER AT FORT KISSIMMEE

02269140 KISSIMMEE RIVER CREST-STAGE GAGE NO3 AT FORT KI

SW 1,911

SW 1,911

02269160 KISSIMMEE RIVER CREST-STAGE GAGE NO4 NR BASINGE

2,035

02269500 REEDY CREEK NR FROSTPROOF

60.9

02269501 REEDY CREEK BELOW CONTROL NR FROSTPROOF

SW

SW

60.9

02269720 MORGAN HOLE CREEK NR AVON PARK

02269830 LITTLE RED WATER LAKE OUTLET NR SEBRING

13.9

9.62

02269997 CARTER CREEK NR, LAKEMONT

02270000 CARTER CREEK NR SEBRING

02270500 ARBUCKLE CREEK NR DE SOTO CITY

ARBUCKLE CREEK AT MOUTH

02270701 LAKE ANNIE OUTLET NR LAKE PLACID
28/09/11 81/33/48 DAVENPORT

$27 / 57 / 40 \quad 81 / 29 / 48$ HESPERIDES

105

28/00/4381/25/29

27/52/24 81/23/52 LAKE WEOHYAKAPKA 105

$27 / 48 / 1481 / 11 / 53$ LAKE MARIAN SW 097

$27 / 48 / 1481 / 11 / 53$ LAKE MARIAN SW 097

27/46/13 81/10/45 LAKE MARIAN SW 105

27/40/15 81/08/42 FORT KISSIMMEE NW 105

27/37/08 81/08/44 FORT KISSIMMEE 055

27/35/27 81/09/20 FORT KISSIMMEE 055

27/35/02 81/09/29 FORT KISSIMMEE 055

27/26/04 81/08/31 BASINGER NW 093

27/43/13 81/28/40 LAKE ARBUCKLE 105

27/43/13 81/28/40 LAKE ARBUCKLE 105

27/37/52 81/17/47 LAKE ARBUCKLE NE 055

27/32/48 81/27/13 LK ARBUCKLE SW 055

27/32/00 81/23/25 LK ARBUCKLE 055

27/32/00 81/23/25 LK ARBUCKLE SW 055

27/26/32 81/17/51 LORIDA

055

27/26/03 81/17/08 LORIDA

$27 / 12 / 3881 / 20 / 56$ CHILDS 
DRAINAGE AREAS FOR SELECTEO STREAM SITES IN FLORIDA

SOUTHERN FLORIDA

KISSIMMEE RIVER BASIN

SITE

NUMBER

NAME AND LOCATION

02271000 STEARNS CREEK NR LAKE PLACID

02271001 STEARNS CREEK BELOW CONTROL NR LAKE. PLACIO

02271500 JOSEPHINE CREEK NR DE SOTO CITY

02271541 GRASSY-HUNTLY PIPELINE NR LAKE PLACID

GRASSY CREEK AT MOUTH

02271675 JOSEPHINE CREEK NR ISTOKPOGA

JOSEPHINE CREEK AT MOUTH

$\begin{array}{cc}\text { SITE } & \begin{array}{c}\text { DRAINAGE } \\ \text { AREA } \\ \text { TYPE }\end{array} \\ \text { SQ.MI } \\ \text { SW } & 44.0 \\ \text { SW } & 44.0 \\ \text { SW } & 109 \\ \text { OT } & 5.39 \\ \text { SW } & 18.7 \\ \text { SW } & 143 \\ \text { SW } & 143\end{array}$

DRAINAGE

$\begin{array}{cccc}\text { AREA } & \text { LAT } & \text { LONG } & \\ \text { QUALIFIER } & \text { DG/MI/SD DG/MI/SD } & \text { CNTY } \\ \text { QUADRANGLE NAME } & \text { CODE }\end{array}$

27/19/22 81/25/09 LK JUNE IN WINTER 055

27/19/24 81/25/07 LK JUNE IN WINTER 055 27/22/26 81/23/37 LK JUNE IN WINTER 055 27/16/15 81/20/10 LAKE PLACID 055 27/23/13 81/22/40 SEBRING 27/23/44 81/20/39 LORIDA 055

TAYLOR CREEK BASIN AND INFLOW TO LAKE OKEECHOBEE FROM NORTH

02274000 TAYLOR CREEK NR BASSINGER

02274330 TAYLOR CREEK ABOVE SI NR OKEECHOBEE

02274495 WILLIAMSON DITCH AT S7 NR OKEECHOBEE

02274500 TAYLOR CREEK ABOVE OKEECHOBEE

02275000 TAYLOR CREEK AT OKEECHOBEE

02275500 TAYLOR CREEK NR OKEECHOBEE

TAYLOR CREEK AT MOUTH

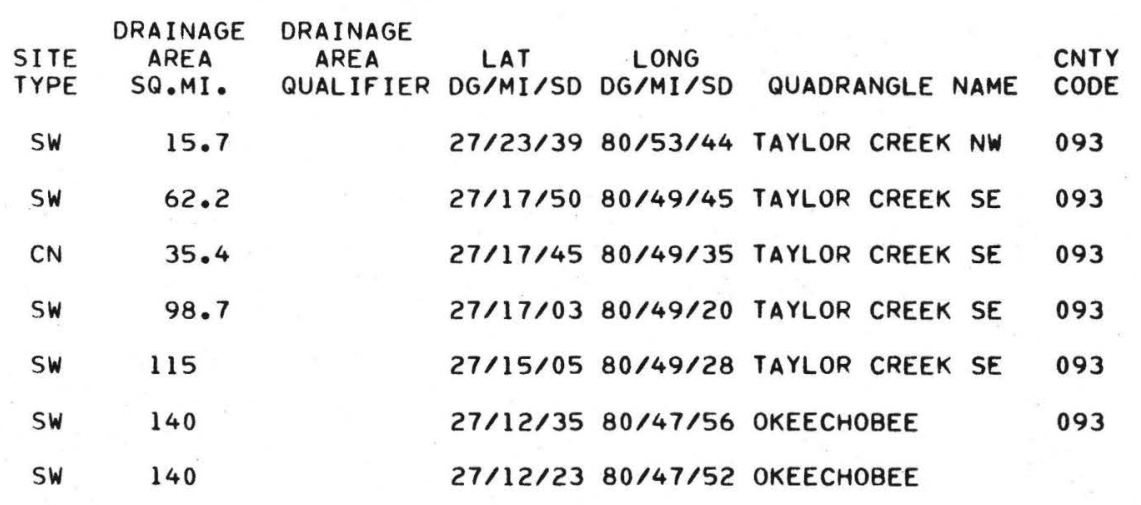


DRAINAGE AREAS FOR SELECTED STREAM SITES IN FLORIDA

SOUTHERN FLORIDA
FISHEATING CREEK BASIN AND INFLOW TO LAKE OKEECHOBEE FROM NORTHWEST

SITE
NUMBER NAME AND LOCATION

02256000 FISHEATING CREEK NR VENUS

02256500 FISHEATING CREEK AT PALMDALE

02256550 FISHEATING CREEK NR PALMDALE

FISHEATING CREEK AT MOUTH

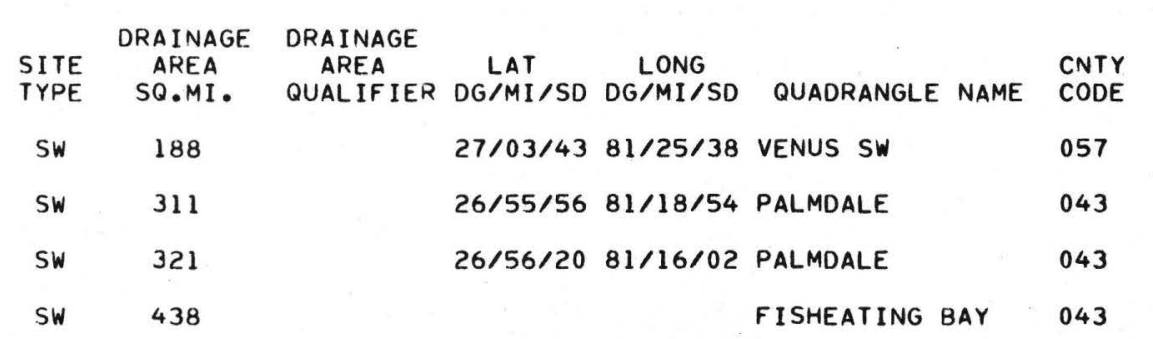

EVERGLADES AND SOUTHEASTERN COASTAL AREA

\begin{abstract}
SITE
NAME AND LOCATION
\end{abstract}

02276568 SAVANNAHS DRAINAGE CANAL AT PORT ST. LUCIE

02276984 MONREVE RANCH DRAINAGE CANAL NR STUART

02277107 WARNER CREEK NEAR JENSEN BEACH, FLA

02279517 LATERAL DITCH NO 1 AT DELRAY BEACH

\begin{tabular}{|c|c|c|c|c|c|c|}
\hline $\begin{array}{l}\text { SITE } \\
\text { TYPE }\end{array}$ & $\begin{array}{c}\text { DRAINAGE } \\
\text { AREA } \\
\text { SQ.MI. }\end{array}$ & $\begin{array}{l}\text { DRAINAGE } \\
\text { AREA } \\
\text { QUALIFIER }\end{array}$ & $\begin{array}{l}\text { LAT } \\
D G / M I / S D\end{array}$ & $\begin{array}{c}\text { LONG } \\
D G / M I / S D\end{array}$ & QUADRANGLE NAME & $\begin{array}{l}\text { CNTY } \\
\text { CODE }\end{array}$ \\
\hline DR & 17.0 & APPROX & $27 / 18 / 12$ & $80 / 18 / 27$ & ANKONA & 111 \\
\hline $\mathrm{CN}$ & 6.20 & & $27 / 03 / 40$ & $80 / 19 / 11$ & INDIAN TOWN SE & 085 \\
\hline SW & 1.46 & & $27 / 14 / 08$ & $80 / 14 / 12$ & ST. LUCIE INLET & 085 \\
\hline $\mathrm{CN}$ & .06 & & $26 / 29 / 01$ & $80 / 03 / 49$ & DELRAY BCH & 099 \\
\hline
\end{tabular}

FLORIDA BAY AND THE FLORIDA KEYS

SITE

02291600 ESTERO RIVER AT ESTERO

02293000 ORANGE RIVER NR FORT MYERS

02293050 ORANGE RIVER AT BUCKINGHAM NR FORT MYERS

ORANGE RIVER AT MOUTH

\begin{tabular}{|c|c|c|c|c|c|c|}
\hline $\begin{array}{l}\text { SITE } \\
\text { TYPE }\end{array}$ & $\begin{array}{l}\text { DRAINAGE } \\
\text { AREA } \\
\text { SQ.MI. }\end{array}$ & $\begin{array}{l}\text { DRAINAGE } \\
\text { AREA } \\
\text { QUAL IF IER }\end{array}$ & $\begin{array}{c}\text { LAT } \\
\mathrm{DG} / \mathrm{MI} / \mathrm{SD}\end{array}$ & $\begin{array}{c}\text { LONG } \\
D G / M I / S D\end{array}$ & QUADRANGLE NAME & $\begin{array}{l}\text { CNTY } \\
\text { CODE }\end{array}$ \\
\hline SW & 50.0 & APPROX & $26 / 26 / 04$ & $81 / 48 / 18$ & ESTERO & 071 \\
\hline SW & 60.0 & APPROX & $26 / 40 / 00$ & $81 / 43 / 00$ & OLGA & 071 \\
\hline SW & 70.0 & APPROX & $26 / 40 / 12$ & $81 / 43 / 56$ & OLGA & 071 \\
\hline SW & 90.0 & APPROX & & & FORT MYERS & 071 \\
\hline
\end{tabular}


SITE

NAME AND LOCATION

02293450 GUM LAKE MARSH OUTLET AT LAKE ALFRED

02293498 SMART-FANNIE CANAL AT STR P-6 NR WINTER HAVEN

02293650 LAKE HENRY OUTLET AB STR P5 NR HAINES CITY

02293664 LAKE HAMILTON OUTLET AT STRUCT P8 NR LK HAMILTO 02293665 LAKE HAMILTON OUTLET BELOW ST P8 NR LAKE HAMIL

02293694 PEACE CREEK DRAINAGE CANAL NR DUNDEE

02293986 PEACE CREEK DRAINAGE CANAL NR ALTURAS

02294068 LAKE LULU OUTLET AT ELOISE

02294490 SADDLE CREEK AB STRUCTURE PII NR BARTOW

02294491 SADDLE CREEK AT S P11 NR BARTOW

SADOLE CREEK AT MOUTH

02294650 PEACE RIVER AT BARTOW

02294898 PEACE RIVER AT FORT MEADE

02295013 BOWLEGS CREEK NR FORT MEADE

02295067 BOWLEGS CREEK AT PISGAH ROAD NR FORT MEADE

BOWLEGS CREEK AT MOUTH

02295356 PAYNE CREEK NR FORT GREEN SPRINGS

02295420 PAYNE CREEK NR BOWLING GREEN

PAYNE CREEK AT MOUTH

02295435 HOG BRANCH NR WAUCHULA

02295637 PEACE RIVER AT ZOLFO SPRINGS

02296180 LITTLE CHARLEY BOWLEGS CREEK NR CREWSVILLE.

02296191 LITTLE CHARLIE BOWLEGS C AT S FENCE LINE RD NR

02296207 LITTLE CHARLEY BOWLEGS CREEK AT COTTAGE RD NR S

02296215 TIGER BRANCH NR SEBRING
DRAINAGE DRAINAGE

AREA LAT LONG

$\begin{array}{lcc}\text { SITE AREA AREA } & \text { LAT } \\ \text { TYPE SQ.MI } & \text { QUALIFIER DG/MI/SO DG/MI/SO QUADRANGLE NAME CODE }\end{array}$

OT 4.2 APPROX 28/06/11 81/41/22 WINTER HAVEN 105

CN $18.0 \quad 28 / 03 / 2081 / 42 / 10$ WINTER HAVEN 105

$\begin{array}{llll}\text { CN } & 4.17 & 28 / 04 / 50 & 81 / 39 / 45 \\ \end{array}$

CN 20.5 28/01/54 81/38/42 WINTER HAVEN 105

CN 20.5 28/01/54 81/38/42 WINTER HAVEN 105

$\begin{array}{llll}\text { CN } & 58 \text { APPROX 28/01/50 81/39/35 WINTER HAVEN } 105\end{array}$

CN $160 \quad 27 / 55 / 2381 / 42 / 28$ ELOISE 105

$\begin{array}{llll}\text { CN } 23 & \text { APPROX } 27 / 59 / 03 & 81 / 43 / 47 \text { ELOISE } & 105\end{array}$

SW $135 \quad 27 / 56 / 1781 / 51 / 05$ BARTOW 105

SW $135 \quad 27 / 56 / 1781 / 51 / 05$ BARTOW 105

SW $\quad 145$

SW $\quad 390$

SW $\quad 465$

SW $\quad 47.2$

SW $\quad 70.9$

SW $\quad 70.9$

SW $\quad 69.4$

SW 121

SW $\quad 123$

SW $\quad 5.31$

SW $\quad 826$

SW $\quad 21.2$

SW $\quad 30.6$

SW $\quad 38.1$

SW $\quad 2.18$

27/55/02 81/49/19 BARTOW

27/54/07 81/49/03 BARTOW

27/45/04 81/46/56 HOMELAND 105

27/41/57 81/41/40 BEREAH 105

$27 / 43 / 1581 / 47 / 20$ BOWLING GREEN 105

27/43/16 81/47/28 BOWLING GREEN

$27 / 36 / 38$ 81/52/17 WAUCHULA

$27 / 37 / 1381 / 49 / 33$ WAUCHULA

29/37/08 81/48/07 WAUCHULA

27/35/32 81/49/20 WAUCHULA

27/30/15 81/48/04 WAUCHULA

049

27/25/48 81/33/04 CREWSVILLE 055

$27 / 27 / 48 \quad 81 / 33 / 15$ CREWSVILLE 055

27/28/13 81/33/14 CREWSVILLE 55

27/28/36 81/31/53 CREWSVILLE 055 
SITE
NUMBER

NAME AND LOCATION

02296222 LITTLE CHARLEY BOWLEGS CREEK AB CONTROL NR SEBR

02296223 LITTLE CHARLEY BOWLEGS CREEK NR SEBRING

OAK CREEK AT MOUTH

02296408 CHARLIE CREEK NR ZOLFO SPRINGS

02296500 CHARLIE CREEK NR GARDNER

CHARLIE CREEK AT MOUTH

02296750 PEACE RIVER AT ARCADIA

02297100 JOSHUA CREEK AT NOCATEE

JOSHUA CREEK AT MOUTH

02297155 HORSE CREEK NR MYAKKA HEAD

02297251 HORSE CREEK NR LIMESTONE

02297310 HORSE CREEK NR ARCADIA

HORSE CREEK AT MOUTH

02297340 PEACE RIVER NR FORT ODGEN

02297733 MOSSY GULLY TRIBUTARY AT STATE HWY 70 NR ARCADI

02297875 COW SLOUGH NR ARCADIA

02298123 PRAIRIE CREEK NR FORT OGDEN

PRAIRIE CREEK AT MOUTH

02298202 SHELL CREEK NR PUNTA GORDA

SHELL CREEK AT MOUTH

PEACE RIVER AT MOUTH

02298523 OGLEBY CREEK NR MYAKKA CITY

\section{DRAINAGE DRAINAGE}

AREA AREA

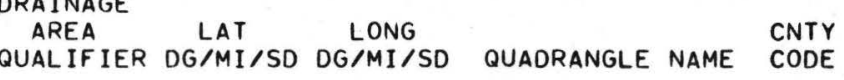

TYPE SQ.MI. QUALIFIER DG/MI/SD DG/MI/SD QUADRANGLE NAME CODE

$41.9 \quad 27 / 28 / 4081 / 33 / 25$ CREWSVILLE 055

41.9 27/28/40 81/33/25 CREWSVILLE

055

27/25/44 81/44/02 SWEETWATER

27/24/33 81/44/46 SWEETWATER

049

27/22/29 81/47/48 GARDNER

049

27/20/58 81/49/34 GARDNER

$27 / 13 / 1981 / 52 / 34$ NOCATEE

027

27/09/59 81/52/47 NOCATEE

027

27/09/44 81/54/06 NOCATEE

APPROX 27/29/13 82/01/25 MYAKKA HEAD

049

27/21/58 81/58/25 LIMESTONE 049

27/11/57 81/59/19 NOCATEE 027

27/06/14 81/59/02 FT OGDEN

27/05/21 81/58/51 FT OGOEN

027

$27 / 12 / 3181 / 40 / 41$ LONG ISL MARSH NW 027

$27 / 12 / 3281 / 34 / 33$ LONG ISL MARSH NE 027

27/03/06 81/47/05 ARCADIA SE

027

26/59/02 81/55/54 CLEVELAND

26/59/04 81/56/09 CLEVELAND

015

26/57/54 81/59/39 CLEVELAND

26/55/22 82/06/46 PUNTA GORDA

27/22/47 81/14/08 MYAKKA CITY

081 
DRAINAGE AREAS FOR SELECTED STREAM SITES IN FLORIDA

PEACE, WITHLACOOCHEE, HILLSBOROUGH RIVERS \& WESTERN COASTAL AREAS

SITE

NAME AND LOCATION

02298458 MYAKKA RIVER NR MYAKKA HEAD

02298478 WINGATE CREEK NR MYAKKA HEAD

02298482 JOHNSON CREEK NR MYAKKA HEAD

02298484 WINGATE CREEK NR MYAKKA CITY

02298554 MYAKKA RIVER NR MYAKKA CITY

02298608 MYAKKA RIVER AT MYAKKA CITY

02298805 MYAKKA RIVER BELOW UPPER MYAKKA LAKE NR SARASOT

02298830 MYAKKA RIVER NR SARASOTA

02298850 MYAKKA RIVER BELOW LOWER MYAKKA LAKE NR SARASOT

02298900 MYAKKA RIVER NR LAUREL

\section{MYAKKA RIVER NR VENICE}

02298970 MYAKKA RIVER TRIBUTARY NR VENICE

02299020 MYAKKA RIVER TRIBUTARY NO 2 NR VENICE

02299030 MYAKKA RIVER TRIBUTARY NO 3 NR VENICE

02299035 MYAKKA RIVER TRIBUTARY NO 4 NR VENICE

02299190 DEER PRAIRIE CREEK NR WOODMERE

02299210 DEER PRAIRIE CREEK NR VENUS

DEER PRAIRIE CREEK AT MOUTH

02299225 MYAKKA RIVER TRIBUTARY NO 5 NR VENICE

02299410 BIG SLOUGH CANAL NR MYAKKA CITY

02299455 BIG SLOUGH CANAL AT NORTH PORT CHARLOTTE

02299470 BIG SLOUGH NR MURDOCK

BIG SLOUGH AT MOUTH

MYAKKA RIVER AT MOUTH MYAKKA RIVER BASIN 
SITE
NUMBER NAME AND LOCATION

02293360 SOUTH PRONG ALLIGATOR CREEK NR PUNTA GORDA

SOUTH PRONG ALLIGATOR CREEK AT CNF WITH NORTH P

02293390 NORTH PRONG ALLIGATOR CREEK NR PUNTA GORDA

NORTH PRONG ALLIGATOR CREEK AT CNF WITH SOUTH P

02293400 ALLIGATOR CREEK NR PUNTA GORDA

ALLIGATOR CREEK AT MOUTH

\begin{tabular}{|c|c|c|c|c|c|c|c|}
\hline $\begin{array}{l}\text { SITE } \\
\text { TYPE }\end{array}$ & $\begin{array}{c}\text { DRAINAGE } \\
\text { AREA } \\
\text { SQ.MI. }\end{array}$ & $\begin{array}{l}\text { DRAINAGE } \\
\text { AREA } \\
\text { QUALIF IER }\end{array}$ & $\begin{array}{c}\text { LAT } \\
D G / M I / S D\end{array}$ & $\begin{array}{c}\text { LONG } \\
\mathrm{DG} / \mathrm{MI} / \mathrm{SD}\end{array}$ & QUADRANGLE & NAME & $\begin{array}{l}\text { CNTY } \\
\text { CODE }\end{array}$ \\
\hline SW & 18.1 & & $26 / 53 / 03$ & $81 / 58 / 45$ & CLEVELAND & & 015 \\
\hline SW & 18.8 & & $26 / 53 / 28$ & $81 / 59 / 12$ & CLEVELAND & & \\
\hline SW & 8.46 & & $26 / 53 / 41$ & $81 / 58 / 31$ & CLEVELANO & & 015 \\
\hline SW & 9.21 & & $26 / 53 / 28$ & $81 / 59 / 12$ & CLEVELAND & & \\
\hline SW & 31.1 & & $26 / 53 / 08$ & $82 / 00 / 22$ & PUNTA GORDA & & 015 \\
\hline SW & 44.5 & & $26 / 52 / 47$ & $82 / 03 / 39$ & PUNTA GORDA & & \\
\hline
\end{tabular}

CUASTAL AREA BETWEEN MYAKKA AND MANATEE RIVERS

SITE
NUMBER NAME AND LOCATION

02299661 EAST BRANCH CORAL CREEK NR PLACIDA 02299678 OYSTER CREEK NR ENGLEWOOD 02299700 COW PEN SLOUGH NR BEE RIDGE 02299721 COW PEN SLOUGH NR VENICE 02299728 FOX CREEK NR LAUREL

02299729 SHAKETT CREEK NR LAUREL

02299738 SOUTH CREEK NR OSPREY

02299750 PHILIPPE CREEK NR SARASOTA

02299780 PHILIPPE CREEK NR BEE RIDGE

02299795 NORTH BRANCH CANAL AT SARASOTA

02299800 PHILIPPE CREEK AT SARASOTA

02299805 TRIBUTARY 1 TO PHIL IPPE CREEK AT SARASOTA

02299807 PHILIPPE CREEK AT HAYOEN

PHILIPPE CREEK AT MOUTH

02299861 WALKER CREEK AT SARASOTA

02299864 WHITAKER BAYOU AT SARASOTA

\begin{tabular}{|c|c|c|c|c|c|c|c|}
\hline $\begin{array}{l}\text { SITE } \\
\text { TYPE }\end{array}$ & $\begin{array}{l}\text { DRAINAGE } \\
\text { AREA } \\
\text { SQ.MI. }\end{array}$ & $\begin{array}{l}\text { DRAINAGE } \\
\text { AREA } \\
\text { QUALIF IER }\end{array}$ & $\begin{array}{c}\text { LAT } \\
D G / M I / S D\end{array}$ & $\begin{array}{c}\text { LONG } \\
\mathrm{DG} / \mathrm{MI} / \mathrm{SD}\end{array}$ & QUADRANGLE & NAME & $\begin{array}{l}\text { CNTY } \\
\text { CODE }\end{array}$ \\
\hline SW & 2.73 & & $26 / 52 / 06$ & $82 / 14 / 17$ & PUNTA GORDA & SW & 115 \\
\hline SW & 5.03 & & $26 / 56 / 03$ & $82 / 18 / 11$ & ENGLEWOOD & & 115 \\
\hline$S L$ & 38 & APPROX & $27 / 14 / 56$ & $82 / 23 / 10$ & LAUREL & & 115 \\
\hline SL & 56 & APPROX & $27 / 10 / 00$ & $82 / 23 / 45$ & LAUREL & & 115 \\
\hline SW & 10 & APPROX & $27 / 09 / 54$ & $82 / 25 / 43$ & LAUREL & & 115 \\
\hline SW & 74 & APPROX & $27 / 08 / 14$ & $80 / 26 / 09$ & LAUREL & & 115 \\
\hline SW & 3.00 & & $27 / 10 / 32$ & $82 / 27 / 30$ & LAUREL & & 115 \\
\hline SW & 21.3 & & $27 / 18 / 31$ & $82 / 27 / 06$ & BEE RIDGE & & 115 \\
\hline SW & 31.1 & & $27 / 19 / 22$ & $82 / 28 / 53$ & BEE RIDGE & & 115 \\
\hline $\mathrm{CN}$ & 7.60 & & $27 / 20 / 14$ & $82 / 29 / 48$ & BEE RIDGE & & 115 \\
\hline SW & 45.1 & & $27 / 19 / 20$ & $82 / 30 / 20$ & SARASOTA & & 115 \\
\hline $\mathrm{CN}$ & 2.87 & & $27 / 17 / 28$ & $82 / 30 / 50$ & SARASOTA & & 115 \\
\hline SW & 53.5 & & $27 / 16 / 55$ & $82 / 31 / 19$ & SARASOTA & & 115 \\
\hline SW & 55.4 & APPROX & $27 / 16 / 15$ & $82 / 32 / 17$ & SARASOTA & & \\
\hline SW & 6.00 & APPROX & $27 / 22 / 03$ & $82 / 32 / 40$ & SARASOTA & & 115 \\
\hline ES & 7.00 & APPROX & $27 / 21 / 11$ & $82 / 33 / 07$ & SARASOTA & & 115 \\
\hline
\end{tabular}


SITE

NUMBER

NAME AND LOCATION

02299913 NORTH FORK MANATEE RIVER AT DUETTE

02299920 NORTH FORK MANATEE RIVER NR MYAKKA CITY

02299935 EAST FORK MANATEE RIVER NR MYAKKA CITY

02299950 MANATEE RIVER NR MYAKKA HEAD

02300004 GILLEY CREEK NR RYE

02300010 MANATEE LAKE NR LORRAINE

02300018 GAMBLE CREEK NR PARRISH

GAMBLE CREEK AT MOUTH

02300032 BRADEN RIVER NR LORRAINE

02300035 BRADEN RIVER NR ONECO

02300040 BRADEN RIVER NR BRADENTON

02300042 WARD LAKE NR BRADENTON

02300044 BRADEN RIVER NR ELWOOD PARK

BRADEN RIVER AT MOUTH

MANATEE RIVER AT MOUTH MANATEE RIVER BASIN

\begin{tabular}{|c|c|c|c|c|c|c|c|}
\hline $\begin{array}{l}\text { SITE } \\
\text { TYPE }\end{array}$ & $\begin{array}{l}\text { DRAINAGE } \\
\text { AREA } \\
\text { SQ.MI. }\end{array}$ & $\begin{array}{l}\text { DRAINAGE } \\
\text { AREA } \\
\text { QUALIF IER }\end{array}$ & $\begin{array}{c}\text { LAT } \\
D G / M I / S D\end{array}$ & $\begin{array}{c}\text { LONG } \\
\mathrm{DG} / M I / S D\end{array}$ & QUADRANGLE & NAME & $\begin{array}{l}\text { CNTY } \\
\text { CODE }\end{array}$ \\
\hline SW & 6.29 & & $27 / 35 / 23$ & $82 / 07 / 40$ & KEENTOWN & & 081 \\
\hline SW & 16.2 & & $27 / 31 / 50$ & $82 / 10 / 19$ & KEENTOWN & & 081 \\
\hline SW & 11.4 & & $27 / 13 / 19$ & $82 / 06 / 14$ & DUETTE & & 081 \\
\hline SW & 65.3 & & $27 / 28 / 24$ & $82 / 12 / 41$ & MYAKKA CITY & & 081 \\
\hline SW & 10.2 & & $27 / 30 / 41$ & $82 / 17 / 15$ & RYE & & 081 \\
\hline RE & 123 & & & & VERNA & & 081 \\
\hline \multirow[t]{2}{*}{ SW } & 50.6 & & $27 / 33 / 11$ & $82 / 23 / 24$ & PARRISH & & 081 \\
\hline & 58.8 & & & & PARRISH & & \\
\hline SW & 25.8 & & $27 / 25 / 20$ & $82 / 25 / 00$ & LORRAINE & & 081 \\
\hline SW & 29.8 & & $27 / 25 / 10$ & $82 / 26 / 40$ & LORRAINE & & 081 \\
\hline SW & 59.0 & & $27 / 26 / 01$ & $82 / 29 / 13$ & LORRAINE & & 081 \\
\hline RE & 59.5 & & $27 / 26 / 29$ & $82 / 29 / 09$ & LORRAINE & & 081 \\
\hline SW & 59.9 & & $27 / 26 / 45$ & $82 / 29 / 30$ & LORRAINE & & 081 \\
\hline SW & 86.4 & & $27 / 30 / 16$ & $82 / 31 / 55$ & PALMETTO & & \\
\hline SW & 357 & & $27 / 31 / 41$ & $82 / 38 / 46$ & PALMETTO & & \\
\hline
\end{tabular}

LITTLE MANATEE RIVER BASIN

SITE
NUMBER

NAME AND LOCATION

02300100 LITTLE MANATEE RIVER NR FORT LONESOME

02300200 SOUTH FORK LITTLE MANATEE RIVER NR DUETTE SOUTH FORK LITTLE MANATEE RIVER AT MOUTH 02300500 LITTLE MANATEE RIVER NR WIMAUMA

LITTLE MANATEE RIVER AT MOUTH

\begin{tabular}{|c|c|c|c|c|c|c|}
\hline $\begin{array}{l}\text { SITE } \\
\text { TYPE }\end{array}$ & $\begin{array}{c}\text { DRAINAGE } \\
\text { AREA } \\
\text { SQ.MI. }\end{array}$ & $\begin{array}{l}\text { DRAINAGE } \\
\text { AREA } \\
\text { QUALIFIER }\end{array}$ & $\begin{array}{c}\text { LAT } \\
D G / M I / S D\end{array}$ & $\begin{array}{c}\text { LONG } \\
\mathrm{DG} / \mathrm{MI} / \mathrm{SD}\end{array}$ & QUADRANGLE NAME & $\begin{array}{l}\text { CNTY } \\
\text { CODE }\end{array}$ \\
\hline SW & 32.9 & & $27 / 42 / 16$ & $82 / 11 / 53$ & FT LONESOME & 057 \\
\hline SW & 9.4 & APPROX & $27 / 35 / 25$ & $82 / 10 / 57$ & KEENTOWN & 081 \\
\hline SW & 39 & APPROX & $27 / 43 / 06$ & $82 / 29 / 00$ & WIMAUMA & \\
\hline SW & 150 & & $27 / 40 / 15$ & $82 / 21 / 10$ & WIMAUMA & 057 \\
\hline SW & 222 & & & & RUSKIN & \\
\hline
\end{tabular}


SITE
NUMBER

NAME AND LOCATION

02301000 NORTH PRONG ALAFIA RIVER AT KEYSVILLE

NORTH PRONG ALAFIA R AT CNF WITH SP ALAFIA RIVE

02301300 SOUTH PRONG ALAFIA RIVER NR LITHIA

02301314 MIZELLE CREEK NR KEYSVILLE

SOUTH PRONG ALAFIA R AT CNF WITH NP ALAFIA RIVE

02301350 LITTLE ALAFIA RIVER NR HOPEWELL

02301368 PLEASANT GROVE RESERVOIR AT PLEASANT GROVE

02301370 LITTLE ALAFIA RIVER AT TURKEY CR RD AT PLEASANT

02301376 LITTLE ALAFIA RIVER AT DURANT

02301400 TURKEY CREEK NR DURANT

TURKEY CREEK AT MOUTH

LITTLE ALAFIA RIVER AT MOUTH

02301500 ALAFIA RIVER AT LITHIA

02301638 ALAFIA RIVER AT BELL SHOALS ROAD NR RIVERVIEW

02301680 BELL CREEK NR RIVERVIEW

BUCKHORN CREEK AT MOUTH

02301718 ALAFIA RIVER AT RIVERVIEW

02301721 ALAFIA RIVER AT GIBSONTON

ALIFIA RIVER AT MOUTH

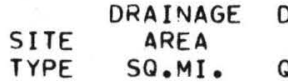

SW $\quad 135$

SW $\quad 140$

SW $\quad 107$

SW $\quad 3.69$

SW $\quad 135$

SW $\quad 8.65$

RE $\quad 19.6$

SW $\quad 19.6$

SW 20.8

SW $\quad 14.2$

SW $\quad 19.0$

SW $\quad 42.7$

SW $\quad 20.1$

SW $\quad 8.27$

SW $\quad 413$

SW $\quad 418$

SW $\quad 420$
SW $\quad 335$

SW $\quad 372$

ORAINAGE

AREA LAT LONG

APPROX 27/53/01 82/06/01 NICHOLS

APPROX 27/51/44 82/08/22

27/47/47 82/07/04 KEYSVILLE

057

$27 / 50 / 14$ 82/05/17 KEYSVILLE

057

$27 / 51 / 44 \quad 82 / 08 / 22$

27/56/15 82/09/23 DOVER

27/54/37 82/10/08 DOVER

057

$27 / 54 / 3482 / 10 / 15$ DOVER

057

27/53/54 82/11/13 DOVER

057

27/56/15 82/11/39 DOVER

057

27/53/54 82/11/17 DOVER

27/53/16 82/11/37 DOVER

APPROX

27/52/19 82/12/41 LITHIA

27/51/25 82/16/10 RIVERVIEW

$27 / 51 / 1282 / 16 / 27$ RIVERVIEW

$27 / 53 / 14 \quad 82 / 18 / 28$ BRANDON

$27 / 51 / 5482 / 18 / 56$ RIVERVIEW

057

27/51/34 82/23/04 GIBSONTON

HILLSBOROUGH RIVER BASIN

\begin{abstract}
SITE
NUMBER
NAME ANO LOCATION
\end{abstract}

02301900 FOX BRANCH NR SOCRUM

FOX BRANCH AT MOUTH

02301990 HILLSBOROUGH RIVER ABOVE CRYSTAL SGS NR ZEPHYRH

$\begin{array}{cclccl}\text { SITE } & \begin{array}{c}\text { DRAINAGE } \\ \text { AREA }\end{array} & \begin{array}{l}\text { DRAINAGE } \\ \text { AREA }\end{array} & \begin{array}{c}\text { LAT } \\ \text { QUALIFIER }\end{array} \text { DG/MI/SD DG/MI/SD } & \text { LUA } \\ \text { TYPE } & \begin{array}{c}\text { SQMI. } \\ \text { QUADANGLE NAME }\end{array} & \begin{array}{c}\text { CNTY } \\ \text { CODE }\end{array} \\ \text { SW } & 9.5 & \text { APPROX } & 28 / 10 / 5582 / 00 / 45 \text { SOCRUM } & 105 \\ \text { SW } & 22 & \text { APPROX } & 28 / 14 / 2082 / 06 / 57 & \text { SOCRUM } & \\ \text { SW } & 82 & \text { APPROX } & 28 / 11 / 0782 / 11 / 03 & \text { ZEPHYRHILLS } & 101\end{array}$


SITE

NUMBER

NAME AND LOCATION

02302100 BIG DITCH NR CRYSTAL SPRINGS

BIG DITCH AT MOUTH

ITCHEPACKESASSA CREEK AT MOUTH

02302500 BLACKWATER CREEK NR KNIGHTS

BLACK WATER CREEK AT MOUTH

02303000 HILLSBOROUGH RIVER NR ZEPHYRHILLS

02303100 NEW RIVER NR ZEPHYRHILLS

NEW RIVER AT MOUTH

TWO HOLE BRANCH AT MOUTH

02303130 BUSY BRANCH NR ZEPHYRHILLS

BUSY BRANCH AT MOUTH

HOLLOMANS BRANCH AT MOUTH

02303167 SPARTMAN BRANCH NR PLANT CITY

02303183 MILL CREEK AT THONOTOSASSA ROAD NR PLANT CITY

02303188 MILL CREEK AT FORBES ROAD NR PLANT CITY

02303200 PEMBERTON CREEK NR DOVER

02303254 BAKER CREEK TRIBUTARY CANAL AT US HWY 92 NR SEF

02303271 BAKER CREEK NR THONOTOSASSA

02303300 FLINT CREEK NR THONOTOSASSA

CAMPBELL CREEK AT MOUTH

02303320 FLINT CREEK AT STATE HIGHWAY 582 NR THONOTOSASS

FLINT CREEK AT MOUTH

02303330 HILLSBOROUGH RIVER AT MORRIS BRIDGE NR THONOTOS

CLAY GULLY AT MOUTH

02303350 TROUT CREEK NR SULPHUR SPRINGS

TROUT CREEK AT MOUTH

\begin{tabular}{|c|c|c|c|c|c|c|}
\hline $\begin{array}{l}\text { SITE } \\
\text { TYPE }\end{array}$ & $\begin{array}{l}\text { DRAINAGE } \\
\text { AREA } \\
\text { SQ.MI. }\end{array}$ & $\begin{array}{l}\text { DRAINAGE } \\
\text { AREA } \\
\text { QUALIFIER }\end{array}$ & $\begin{array}{c}\text { LAT } \\
\mathrm{DG} / \mathrm{MI} / \mathrm{SD}\end{array}$ & $\begin{array}{c}\text { LONG } \\
\mathrm{DG} / \mathrm{MI} / \mathrm{SD}\end{array}$ & QUADRANGLE NAME & $\begin{array}{l}\text { CNTY } \\
\text { CODE }\end{array}$ \\
\hline $\mathrm{CN}$ & 1.6 & APPROX & $28 / 09 / 35$ & $82 / 09 / 10$ & ZEPHYRHILLS & 057 \\
\hline SW & 5.5 & APPROX & $28 / 10 / 13$ & $82 / 11 / 40$ & ZEPHYHILLS & \\
\hline SW & 60 & APPROX & $28 / 08 / 15$ & $82 / 07 / 07$ & SOCRUM & 057 \\
\hline SW & 110 & APPROX & $28 / 08 / 25$ & $82 / 09 / 00$ & ZEPHYRHILLS & 057 \\
\hline SW & 120 & APPROX & $28 / 09 / 02$ & $82 / 12 / 56$ & ZEPHYRHILLS & \\
\hline SW & 220 & APPROX & $28 / 08 / 59$ & $82 / 13 / 57$ & ZEPHYRHILLS & 057 \\
\hline SW & 15 & APPROX & $28 / 09 / 55$ & $82 / 15 / 55$ & WESLEY CHAPEL & 057 \\
\hline SW & 19 & APPORX & $28 / 08 / 37$ & $82 / 15 / 01$ & WESLEY CHAPEL & \\
\hline SW & 11 & APPROX & $28 / 08 / 14$ & $82 / 15 / 42$ & WESLEY CHAPEL & \\
\hline SW & 11 & APPROX & $28 / 08 / 48$ & $82 / 16 / 48$ & WESLEY CHAPEL & 057 \\
\hline SW & 14 & APPROX & $28 / 07 / 18$ & $82 / 16 / 19$ & THONOTOSASSA & \\
\hline SW & 14 & APPROX & $28 / 05 / 53$ & $82 / 16 / 14$ & THONOTOSASSA & \\
\hline SW & 8.2 & APPROX & $28 / 01 / 32$ & $82 / 11 / 13$ & PLANT CITY WEST & 057 \\
\hline SW & 7.8 & APPROX & $28 / 02 / 08$ & $82 / 09 / 51$ & PLANT CITY WEST & 057 \\
\hline Sw & 9.10 & & $28 / 01 / 50$ & $82 / 11 / 14$ & PLANT CITY WEST & 057 \\
\hline SW & 24 & APPROX & $28 / 01 / 34$ & $82 / 14 / 12$ & PLANT CITY WEST & 057 \\
\hline SW & 24 & APPROX & $28 / 00 / 47$ & $82 / 15 / 25$ & THONOTOSASSA & 057 \\
\hline SW & 58 & APPROX & $28 / 02 / 52$ & $82 / 16 / 04$ & THONOTOSASSA & 057 \\
\hline SW & 60 & APPROX & $28 / 04 / 04$ & $82 / 16 / 04$ & THONOTOSASSA & 057 \\
\hline SW & 8.5 & APPROX & $28 / 04 / 03$ & $82 / 15 / 51$ & & \\
\hline SW & 70 & APPROX & $28 / 04 / 30$ & $82 / 15 / 50$ & THONOTOSASSA & 057 \\
\hline SW & 71 & APPROX & $28 / 04 / 57$ & $82 / 17 / 07$ & THONOTOSASSA & \\
\hline SW & 375 & APPROX & $28 / 05 / 50$ & $82 / 18 / 45$ & THONOTOSASSA & 057 \\
\hline SW & 8.3 & APPROX & $28 / 06 / 10$ & $82 / 19 / 20$ & THONOTOSASSA & \\
\hline SW & 23 & APPROX & $28 / 08 / 20$ & $82 / 21 / 50$ & WESLEY CHAPEL & 057 \\
\hline SW & 31 & $0 x$ & 28 & $82 / 19 / 20$ & THONOTOSASSA & \\
\hline
\end{tabular}


SITE

NAME AND LOCATION

02303358 CYPRESS CREEK NR DARBY

02303400 CYPRESS CREEK NR SAN ANTONIO

02303408 CYPRESS CREEK NR DREXEL

02303420 CYPRESS CREEK AT WORTHINGTON GARDENS

02303433 HANNA LAKE OUTLET NR LUTZ

02303512 THIRTEENMILE RUN NR DREXEL

02303790 THIRTEENMILE RUN NR LUTZ

THIRTEENMILE RUN AT MOUTH

02303800 CYPRESS CREEK NR SULPHUR SPRINGS

CYPRESS CREEK AT MOUTH

02304000 HILLSBOROUGH RIVER AT FOWLER AVENUE NR TAMPA

02304040 HILLSBOROUGH RIVER AT WHITEWAY AVENUE

02304500 HILLSBOROUGH RIVER NR TAMPA

02304501 HILLSBOROUGH RIVER BELOW DAM NR TAMPA

02305000 LAKE HUTCHINS OUTLET NR LUTZ

02305176 LAKE CRENSHAW OUTLET NR LUTZ

02305275 BRANT-CHARLES CANAL NR LUTZ

02305312 BIRD-PLATT CANAL NR LUTZ

02305500 DRAINAGE DITCH AT BEARSS AVENUE NR SULPHUR SPRI

02305800 DRAINAGE D AT FLA AVE AND ATLANTIC BLVD NR S SG

02306002 ARTIC ST STORM DRAIN AT SULPHUR SPRINGS, FLA.

02306006 KIRBY STREET DRAINAGE OITCH AT TAMPA

02306014 HILLSBOROUGH RIVER AT SLIGH AVENUE AT TAMPA

02306021 ST LOUIS STREET DRAINAGE DITCH AT TAMPA

HILLSBOROUGH RIVER AT MOUTH

\section{DRAINAGE DRAINAGE}

SITE AREA AREA LAT LONG 4 CNTY$$
7.11
$$

SW $\quad 7.11$

SW $\quad 73.2$

SW 117

OT

$$
\text { - }
$$

.7 APPROX

7.2 APPROX

2.8 APPROX

4.0 APPROX

160 APPROX

165 APPROX

630 APPROX

630

650

650

650

2.7 APPROX

1.3 APPROX

.9 APPROX

7.8 APPROX

12 APPROX

14 APPROX
28/22/32 82/19/47 SPRING LAKE

28/19/25 82/23/03 EHREN

28/16/43 82/24/35 EHREN

28/11/08 82/24/03 LUTZ

28/08/10 82/26/35 LUTZ

28/11/08 82/27/07 LUTZ

$28 / 06 / 3382 / 25 / 52$ SULPHUR SPGS

$28 / 06 / 0682 / 24 / 47$ SULPHUR SPRINGS

$28 / 05 / 2082 / 24 / 33$ SULPHUR SPGS

$28 / 04 / 2682 / 22 / 51$ SULPHUR SPRINGS

28/03/15 82/21/50 THONOTOSASSA

28/02/44 82/22/01 THONOTOSASSA

28/01/25 82/25/41 SULPHUR SPGS

28/01/26 82/25/42 SULPHUR SPGS

28/07/40 82/29/15 LUTZ

$28 / 07 / 2582 / 29 / 49$

$28 / 07 / 0682 / 28 / 40$ SULPHUR SPGS

28/05/55 82/28/37 SULPHUR SPGS

$28 / 05 / 1682 / 27 / 55$ SULPHUR SPGS

28/03/55 82/27/34 SULPHUR SPGS

$28 / 01 / 4382 / 27 / 22$ SULPHUR SPGS

28/01/07 82/28/04 SULPHUR SPGS

0/00/00 0/00/00 SULPHUR SPGS

27/57/54 82/29/13 TAMPA

TAMPA
101

101

101

101

057

101

057

057

057

057

057

057

057

057

057

057

057

057

057

057

057 
SITE
NUMBER

NAME AND LOCATION

02300700 BULLFROG CREEK NR WIMAUMA

02300703 BULLFROG CREEK NR RIVERVIEW

02300705 BULLFROG CREEK AT GIBSONTON

?ULLFROG CREEK AT MOUTH

02301780 SIXMILE CREEK AT BUFFALO AVENUE NR TAMPA

OC. $? 83$ MANGO LAKE NUTLET AT MANGO

02301800 SIXMILE CREEK AT TAMPA

02301802 SIXMILE CREEK AT S-160 AT TAMPA

PALM RIVER AT MOUTH

$? 306071$ GANDY BOULEVARD DRAINAGE DITCH AT TAMPA

02306240 MAGDALENE BAY CANAL NR SULPHUR SPRINGS

TAMPA BAY AND COASTAL AREAS

02306289 LAKE MAGDALENE OUTLET NR LUTZ

02306500 SWEETWATER CREEK NR SULPHUR SPRINGS

02306647 SWEETWATER CREEK NR TAMPA

SWEETWATER CREEK AT MOUTH

BRUSHY CREEK AT MOUTH

02307000 ROCKY CREEK NR SULPHUR SPRINGS

ROCKY CREEK AT MOUTH

02307243 BROOKER CREEK NR ODESSA

02307323 BROOKER CREEK NR LAKE FERN

02307359 BROOKER CREEK NR TARPON SPRINGS

BRDOKER CREEK AT MOUTH

02307498 LAKE TARPON CANAL AT S-551 NR OLDSMAR

02307499 LAKE TARPON CANAL BELOW S-551 NR OLDSMAR

02307537 SOUTH FORK BISHOP CREEK NR OLDSMAR

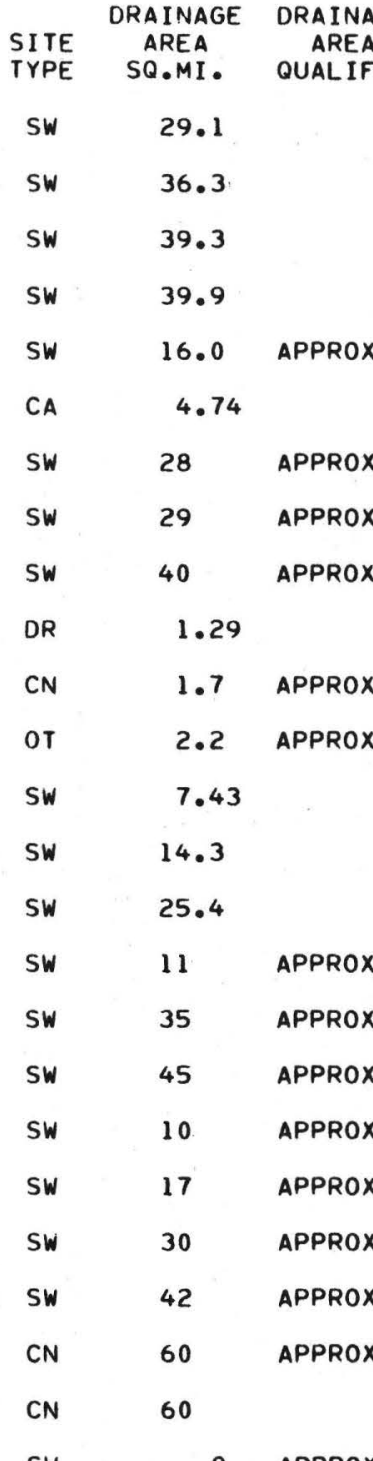

LAT LONG

CNTY

27/47/30 82/21/09 RIVERVIEW

057

27/50/05 82/20/47 RIVERVIEW

057

27/50/15 82/22/56 GIBSONTON

057

27/50/12 82/23/38 GIBSONTON

27/58/52 82/21/17 BRANDON

057

27/58/17 82/18/33 BRANDON

057

27/57/59 82/22/07 BRANDON

057

27/57/21 82/22/15 BRANDON

057

$27 / 56 / 3082 / 24 / 45$ TAMPA

$27 / 53 / 3782 / 31 / 07$ GANDY BRIDGE

$28 / 04 / 3382 / 29 / 32$ SULPHUR SPGS

057

28/04/26 82/30/01 CITRUS PARK

28/02/33 82/30/44 CITRUS PARK

057

057

28/00/55 82/32/31 CITRUS PARK

$27 / 58 / 2282 / 34 / 02$ GANDY BRIDGE

28/03/43 82/33/54 CITRUS PARK

28/02/12 82/34/34 CITRUS PARK

057

$27 / 58 / 5082 / 36 / 04$ GANDY BRIDGES

28/08/50 82/35/40 ODESSA

057

28/08/26 82/38/24 ELFERS

057

28/05/45 82/41/15 OLOSMAR

103

103

103 

TAMPA BAY AND COASTAL AREAS

SITE

NUMBER

NAME AND LOCATION

02307542 NORTH FORK BISHOP CREEK NR OLDSMAR

02307544 BISHOP CREEK NR OLDSMAR

BISHOP CREEK AT MOUTH

02307697 ALLIGATOR CREEK AT SAFETY HARBOR

02307731 ALLEN CREEK NR LARGO

02307768 ALLEN CREEK NR CLEARWATER

ALLEN CREEK AT MOUTH

02308193 BOOKER CREEK AT ST PETERSVILLE

02310212 PECK SINK ORAIN NR BROOKSVILLE

\begin{tabular}{|c|c|c|c|c|c|c|}
\hline $\begin{array}{l}\text { SITE } \\
\text { TYPE }\end{array}$ & $\begin{array}{c}\text { DRAINAGE } \\
\text { AREA } \\
\text { SQ.MI. }\end{array}$ & $\begin{array}{l}\text { DRAINAGE } \\
\text { AREA } \\
\text { QUALIFIER }\end{array}$ & $\begin{array}{c}\text { LAT } \\
D G / M I / S D\end{array}$ & $\begin{array}{c}\text { LONG } \\
\mathrm{DG} / \mathrm{MI} / \mathrm{SD}\end{array}$ & QUADRANGLE NAME & $\begin{array}{l}\text { CNTY } \\
\text { CODE }\end{array}$ \\
\hline SW & 1.0 & APPROX & $28 / 01 / 11$ & $82 / 41 / 21$ & OLDSMAR & 103 \\
\hline SW & 2.02 & & $28 / 01 / 05$ & $82 / 41 / 14$ & OLDSMAR & 103 \\
\hline SW & 2.0 & APPROX & $28 / 01 / 14$ & $82 / 41 / 09$ & OLDSMAR & \\
\hline SW & 9.0 & APPROX & $27 / 58 / 45$ & $82 / 41 / 45$ & SAFETY HARBOR & 103 \\
\hline SW & 1.88 & APPROX & $27 / 56 / 30$ & $82 / 45 / 00$ & SAFETY HARBOR & 103 \\
\hline SW & 7.0 & APPROX & $27 / 56 / 10$ & $82 / 43 / 50$ & SAFETY HARBOR & 103 \\
\hline SW & 7.0 & APPROX & $27 / 56 / 10$ & $82 / 43 / 48$ & SAFETY HARBOR & \\
\hline SW & 3.76 & & $27 / 46 / 23$ & $82 / 39 / 24$ & ST PETERSBURG & 103 \\
\hline DR & 16.6 & & $28 / 32 / 00$ & $82 / 25 / 45$ & BROOKSVILLE & 053 \\
\hline
\end{tabular}

COASTAL. AREA FROM TAMPA BAY TO WITHLACOOCHEE RIVER

\section{SITE
NUMBER NAME AND LOCATION}

02308773 BEAR CREEK AT ST PETERSBURG

02308858 LONG BAYOU AB NORTH DAM NR LARGO

02308889 SEMINOLE LAKE OUTLET NR LARGO

02308929 SAINT JOE CREEK AT ST PETERSBURG

02309011 UNNAMED LAKE OUTLET AT ST PETERSBURG

02309043 WALSINGHAM RESERVOIR NR INDIAN ROCKS

02309044 MCKAY C AT WALSINGHAM RESERVOIR NR INDIAN ROCKS

\begin{abstract}
02309058 TAYLOR LAKE AT LARGO
\end{abstract}
02309059 MCKAY CREEK AT TAYLOR LAKE AT LARGO

02309160 TURNER ST STORM DRAIN AT CLEARWATER, FLA

02309258 STEVENSON CREEK AT CLEARWATER

STEVENSON CREEK AT MOUTH

\begin{tabular}{|c|c|c|c|c|c|c|}
\hline $\begin{array}{l}\text { SITE } \\
\text { TYPE }\end{array}$ & $\begin{array}{l}\text { DRAINAGE } \\
\text { AREA } \\
\text { SQ.MI. }\end{array}$ & $\begin{array}{l}\text { DRA INAGE } \\
\text { AREA } \\
\text { QUALIF IER }\end{array}$ & $\begin{array}{c}\text { LAT } \\
\mathrm{DG} / \mathrm{MI} / \mathrm{SD}\end{array}$ & $\begin{array}{c}\text { LONG } \\
\mathrm{DG} / \mathrm{MI} / \mathrm{SD}\end{array}$ & QUADRANGLE NAME & $\begin{array}{l}\text { CNTY } \\
\text { CODE }\end{array}$ \\
\hline SW & 1.89 & & $27 / 46 / 17$ & $82 / 42 / 51$ & ST PETERSBURG & 103 \\
\hline SW & & & $27 / 53 / 10$ & $82 / 46 / 55$ & CLEARWATER & 103 \\
\hline SW & 6.94 & & $27 / 50 / 20$ & $82 / 46 / 50$ & SEMINOLE & 103 \\
\hline DR & 1.72 & & $27 / 48 / 48$ & $82 / 40 / 47$ & ST PETERSBURG & 103 \\
\hline $\mathrm{CN}$ & .18 & & $27 / 47 / 53$ & $82 / 44 / 52$ & ST PETERSBURG & 103 \\
\hline RE & 2.4 & & $27 / 48 / 40$ & $82 / 45 / 35$ & CLEARWATER & 103 \\
\hline SW & 2.4 & & $27 / 52 / 45$ & $82 / 48 / 35$ & CLEARWATER & 103 \\
\hline RE & 4.1 & & $27 / 54 / 25$ & $82 / 48 / 10$ & CLEARWATER & 103 \\
\hline SW & 4.1 & & $27 / 54 / 25$ & $82 / 48 / 10$ & CLEARWATER & 103 \\
\hline DR & .24 & & $28 / 57 / 36$ & $82 / 47 / 56$ & CLEARWATER & 103 \\
\hline SW & 4.88 & & $27 / 58 / 19$ & $82 / 46 / 54$ & CLEARWATER & 103 \\
\hline SW & 6.77 & & $27 / 58 / 58$ & $24 / 71 / 50$ & CLEARWATER & \\
\hline
\end{tabular}


SITE

NUMBER

NAME AND LOCATION

02309421 CURLEW CREEK NR OZONA

CURLEW CREEK AT MOUTH

02309502 INNISBROOK CANAL NR CRYSTAL BEACH

02309648 ANCLOTE RIVER NR FIVAY JUNCTION

02309848 SOUTH BRANCH ANCLOTE RIVER NR ODESSA

02309900 SOUTH BRANCH ANCLOTE RIVER AT ODESSA SOUTH BRANCH ANCLOTE RIVER AT MOUTH

02310000 ANCLOTE RIVER NR ELFERS

02310150 HOLLIN CREEK TRIBUTARY NR TARPON SPRINGS

HOLLIN CREEK AT MOUTH

02310166 ANCLOTE RIVER NR TARPON SPRINGS

ANCLOTE RIVER AT MOUTH

02310224 SPARKMAN LAKE DUTLET NR MASARYKTOWN

02310240 JUMPING GULLY NR LOYCE

JUMPING GULLY AT MOUTH

02310280 PITHLACHASCOTEE RIVER NR FIVAY JUNCTION 02310285 FIVEMILE CREEK NR FIVAY JUNCTION

FIVEMILE CREEK AT MOUTH

02310300 PITHLACHASCOTEE RIVER NR NEW PORT RICHEY

02310310 PITHLACHASCOTEE RIVER AT PORT RICHEY

PITHLACHASCOTEE RIVER AT MOUTH

02310350 BEAR CREEK NR HUDSON

02310352 BEAR CREEK AT PLAZA DRIVE NR HUDSON

02310355 BEAR CREEK BELOW BEAR SINK NR HUOSON

\begin{tabular}{|c|c|c|c|c|c|c|}
\hline $\begin{array}{l}\text { SITE } \\
\text { TYPE }\end{array}$ & $\begin{array}{l}\text { DRAINAGE } \\
\text { AREA } \\
\text { SQ.MI. }\end{array}$ & $\begin{array}{l}\text { DRAINAGE } \\
\text { AREA } \\
\text { QUALIF IER }\end{array}$ & $\begin{array}{l}\text { LAT } \\
\text { DG/MI/SO }\end{array}$ & $\begin{array}{c}\text { LONG } \\
D G / M I / S D\end{array}$ & QUADRANGLE NAME & $\begin{array}{l}\text { CNTY } \\
\text { CODE }\end{array}$ \\
\hline SW & 3.4 & APPRUX & $28 / 02 / 24$ & $82 / 44 / 51$ & OLDSMAR & 103 \\
\hline SW & 9.8 & APPROX & $28 / 02 / 41$ & $82 / 47 / 11$ & DUNEDIN & \\
\hline $\mathrm{CN}$ & $1.5^{\circ}$ & APPROX & $28 / 06 / 34$ & $82 / 45 / 32$ & DUNEDIN & 103 \\
\hline SW & 9.0 & APPROX & $28 / 15 / 23$ & $82 / 31 / 58$ & FIVAY JUNCTION & 101 \\
\hline SW & 17.1 & & $28 / 11 / 08$ & $82 / 33 / 13$ & ODESSA & 101 \\
\hline SW & 25.3 & & $28 / 12 / 15$ & $82 / 35 / 42$ & ODESSA & 101 \\
\hline SW & 27.8 & & $28 / 13 / 20$ & $82 / 36 / 35$ & ODESSA & \\
\hline SW & 72.5 & & $28 / 12 / 50$ & $82 / 40 / 00$ & ELFERS & 101 \\
\hline SW & 2.1 & APPROX & $28 / 09 / 48$ & $82 / 42 / 46$ & ELFERS & 103 \\
\hline SW & 3.4 & APPROX & $28 / 09 / 51$ & $82 / 43 / 29$ & ELFERS & \\
\hline SW & 104 & & $28 / 09 / 24$ & $82 / 44 / 49$ & ELFERS & 103 \\
\hline SW & 113 & & $28 / 10 / 31$ & $82 / 47 / 32$ & TARPON SPRINGS & \\
\hline SW & 11.6 & & $28 / 27 / 23$ & $82 / 22 / 14$ & SPRING LAKE & 053 \\
\hline SW & 43 & APPROX & $28 / 23 / 06$ & $82 / 29 / 22$ & MASARYKTOWN & 101 \\
\hline OT & 45 & APPROX & $28 / 22 / 44$ & $82 / 31 / 03$ & PORT RICHEY NE & \\
\hline SW & 150 & & $28 / 19 / 44$ & $82 / 32 / 13$ & FIVAY JUNCTION & 101 \\
\hline SW & 7.05 & & $28 / 17 / 20$ & $82 / 31 / 50$ & FIVAY JUNCTION & 101 \\
\hline SW & 9.02 & & $28 / 17 / 44$ & $82 / 34 / 00$ & FIVAY JUNCTION & \\
\hline SW & 182 & & $28 / 15 / 19$ & $82 / 39 / 37$ & PORT RICHEY & 101 \\
\hline SW & 195 & & $28 / 16 / 10$ & $82 / 43 / 36$ & PORT RICHEY & 101 \\
\hline SW & 196 & & $28 / 16 / 34$ & $82 / 44 / 37$ & PORT RICHEY & \\
\hline SW & 22.0 & & $28 / 19 / 10$ & $82 / 39 / 06$ & PORT RICHEY & 101 \\
\hline SW & 29.2 & & $28 / 19 / 38$ & $82 / 39 / 59$ & PORT RICHEY & 101 \\
\hline SW & 29.7 & & $28 / 19 / 50$ & $82 / 40 / 22$ & PORT RICHEY & 101 \\
\hline
\end{tabular}



WITHLACOOCHEE RIVER BASIN

SITE
NUMBER

NAME AND LOCATION

02310787 WITHLACOOCHEE RIVER NR POYNER

02310800 WITHLACOOCHEE RIVER NR EVA

02310900 PONY CREEK NR POLK CITY

02310931 WITHLACOOCHEE RIVER NR ROCK RIDGE

02310944 WITHLACOOCHEE RIVER AT CEDAR FORD NR CUMPRESSCO

02310947 WITHLACOOCHEE RIVER NR CUMPRESSCO

02310995 GATOR CREEK NR RICHLAND

GATOR CREEK AT MOUTH

02311005 WITHLACOOCHEE RIVER NR RICHLAND

02311500 WITHLACOOCHEE RIVER NR DADE. CITY

02311700 DAOE CITY CANAL NR DADE CITY

02311750 DADE CITY CANAL AT MUD LAKE NR DADE CITY

02311787 WITHLACOOCHEE RIVER NR LACOOCHEE

02311836 DEVILS CREEK NR LACOOCHEE

02312000 WITHLACOOCHEE RIVER AT TRILBY

02312140 BAYROOT SLOUGH HEADWATERS NR BAY LAKE

02312145 MILL CREEK NR CARTERS ISLAND

02312180 LITTLE WITHLACOOCHEE RIVER NR TARRYTOWN

02312194 BIG GANT CANAL AT STRUCTURE S-11 NR WEBSTER

02312197 BIG GANT CANAL AT STRUCTURE WC-2 AT RERDELL

02312198 BIG GANT CANAL BELOW STRUCTURE WC-2 AT RERDELL

02312200 LITTLE WITHLACOOCHEE RIVER AT RERDELL

LITTLE WITHLACOOCHEE RIVER AT MOUTH

02312500 WITHLACOOCHEE RIVER AT CROOM

02312530 BLUE SINK DRAIN NR BROOKSVILLE

\begin{tabular}{|c|c|c|c|c|c|c|}
\hline $\begin{array}{l}\text { SITE } \\
\text { TYPE }\end{array}$ & $\begin{array}{l}\text { DRAINAGE } \\
\text { AREA } \\
\text { SQ.MI. }\end{array}$ & $\begin{array}{l}\text { DRAINAGE } \\
\text { AREA } \\
\text { QUALIF IER }\end{array}$ & $\begin{array}{c}\text { LAT } \\
\mathrm{DG} / \mathrm{MI} / \mathrm{SD}\end{array}$ & $\begin{array}{c}\text { LONG } \\
\text { DG/MI/SD }\end{array}$ & QUADRANGLE NAME & $\begin{array}{l}\text { CNTY } \\
\text { CODE }\end{array}$ \\
\hline SW & 16 & APPROX & $28 / 18 / 21$ & $81 / 47 / 36$ & POYNER & 105 \\
\hline Sw & 130 & APPROX & $28 / 21 / 38$ & $81 / 49 / 08$ & POYNER & 105 \\
\hline SW & 9.5 & APPROX & $28 / 15 / 03$ & $81 / 48 / 51$ & POYNER & 105 \\
\hline SW & 235 & APPROX & $28 / 19 / 32$ & $81 / 55 / 56$ & ROCK RIDGE & 105 \\
\hline SW & 260 & APPROX & $28 / 19 / 20$ & $82 / 00 / 24$ & BRANCHBOROUGH & 119 \\
\hline Sw & 280 & APPROX & $28 / 18 / 42$ & $82 / 03 / 22$ & BRANCHBOROUGH & 101 \\
\hline SW & 80 & APPROX & $28 / 18 / 08$ & $82 / 03 / 22$ & BRANCHBOROUGH & 101 \\
\hline SW & 80 & APPROX & $28 / 18 / 14$ & $82 / 03 / 35$ & BRANCHBOROUGH & 101 \\
\hline SW & 365 & APPROX & $28 / 17 / 09$ & $82 / 06 / 24$ & BRANCHBOROUGH & 101 \\
\hline SW & 390 & APPROX & $28 / 21 / 08$ & $82 / 07 / 34$ & DADE CITY & 101 \\
\hline $\mathrm{CN}$ & 35 & APPROX & $28 / 22 / 55$ & $82 / 10 / 48$ & LACOOCHEE & 101 \\
\hline CN & 40 & APPROX & $28 / 24 / 26$ & $82 / 09 / 55$ & LACOOCHEE & 101 \\
\hline SW & 465 & APPROX & $28 / 26 / 33$ & $82 / 09 / 48$ & LACOOCHEE & 101 \\
\hline SW & 35 & APPROX & $28 / 25 / 50$ & $82 / 05 / 11$ & CLAY SINK & 101 \\
\hline Sw & 570 & APPROX & $28 / 28 / 47$ & $82 / 10 / 40$ & LACOOCHEE & 053 \\
\hline SL & 18 & APPROX & $28 / 27 / 23$ & $81 / 55 / 14$ & BAY LAKE & 069 \\
\hline SW & 17 & APPROX & $28 / 29 / 40$ & $81 / 54 / 35$ & BAY LAKE & 069 \\
\hline SW & 85.0 & & $28 / 31 / 17$ & $82 / 03 / 18$ & WEBSTER & 119 \\
\hline $\mathrm{CN}$ & 18 & APPROX & $28 / 34 / 47$ & $82 / 05 / 45$ & WEBSTER & 119 \\
\hline $\mathrm{CN}$ & 30 & APPROX & $28 / 34 / 16$ & $82 / 08 / 52$ & SAINT CATHERINE & 119 \\
\hline $\mathrm{CN}$ & 30 & & $28 / 34 / 16$ & $82 / 08 / 54$ & SAINT CATHERINE & 119 \\
\hline SW & 145 & APPROX & $28 / 34 / 21$ & $82 / 09 / 20$ & SAINT CATHERINE & 053 \\
\hline SW & 150 & APPROX & $28 / 34 / 22$ & $82 / 12 / 01$ & SAINT CATHERINE & \\
\hline SW & 810 & & $28 / 35 / 33$ & $82 / 13 / 20$ & SAINT CATHERINE & 053 \\
\hline DR & 68.4 & & $28 / 36 / 30$ & $82 / 20 / 21$ & BROOKSVILLE SE & 053 \\
\hline
\end{tabular}


SITE

NUMBER

NAME AND LOCATION

02312600 WITHLACOOCHEE RIVER NR FLORAL CITY

02312625 JUMPER CREEK CANAL NR CENTER HILL

02312630 JUMPER CREEK CANAL AT CENTER HILL

02312632 JUMPER CREEK CANAL NR BEVILLES CORNERS

02312635 JUMPER CREEK CANAL NR SUMTERVILLE

02312640 JUMPER CREEK CANAL NR BUSHNELL

02312645 JUMPER CREEK CANAL NR WAHOO

JUMPER CREEK AT MOUTH

02312667 SHADY BROOK NR SUMTERVILLE

02312685 WALLED SINK DRAIN NR COLEMAN

02312700 OUTLET RIVER AT PANACOOCHEE RETREATS

OUTLET RIVER AT MOUTH

02312719 WITHLACOOCHEE RIVER ABOVE WYSONG DAM AT CARLSON 02312720 WITHLACOOCHEE RIVER AT WYSONG DAM NR CARLSON 02313000 WITHLACOOCHEE RIVER NR HOLDER

02313180 BLUE RUN AT DUNNELLON

BLUE RUN AT MOUTH

02313200 WITHLACOOCHEE RIVER AT DUNNELLON

02313229 ROUSSEAU LAKE NR DUNNELLON

DRAINAGE DRAINAGE

AREA AREA LAT LONG

AREA LAT LONG CNTY

TYPE SQ.MI. QUALIFIER DG/MI/SD DG/MI/SD QUADRANGLE NAME CODE

SW 28/44/36 82/13/13 WATTOO 017

$\begin{array}{lll}\text { CN } 6.72 \quad 28 / 37 / 03 & 81 / 59 / 39 \text { MASCOTTE } & 119\end{array}$

CN $13.6 \quad 28 / 38 / 58 \quad 82 / 00 / 19$ BUSHNELL

119

SW $\quad 15.4$

28/40/04 82/01/05 BUSHNELL

28/41/46 82/03/18 BUSHNELL

119

CN $\quad 28.6$

CN $40 \quad$ APPROX

28/41/45 82/06/34 BUSHNELL

119

SW $\quad 50.6$

SW $\quad 83 \quad$ APPROX

$28 / 42 / 15$ 82/09/26 WAHOO

28/45/34 82/11/10 RUTLAND

SW 8.0. APPROX

28/46/10 82/04/00 CLERMONT WEST

119

DR 90. APPROX

28/45/05 82/02/05 WILDWOOD

119

SW 38. APPROX

28/48/40 81/58/50 LEESBURG WEST 119

SW 420. APPROX

28/49/01 82/08/40 RUTLAND

119

SW 425. APPROX

28/47/50 82/09/56 RUTLAND

28/49/22 82/10/58 RUTLAND

119

SW 1.520. APPROX

28/49/23 82/11/00 RUTLAND

119

SW 1,825. APPROX

$28 / 59 / 1982 / 20 / 59$ STOKES FERRY

083

SW

47.8 NONCON

29/02/57 82/26/53 DUNNELLON

DUNNELLON

SW $1,960 \quad$ APPROX

29/02/45 82/27/53 DUNNELLON

29/00/36 82/37/00 YANKEETOWN SE
083

083

075 
DRAINAGE AREAS FOR SELECTED STREAM SITES IN FLORIDA

SUWANNEE AND AUCILLA RIVERS

WACCASASSA RIVER AND COASTAL AREA BETWEEN WITHLACOOCHEE AND SUWANNEE RIVERS

SITE

NAME ANO LOCATION

02313400 WACCASASSA RIVER NR BRONSON

02313500 WACCASASSA RIVER NR OTTER CREEK

02313700 WACCASASSA RIVER NR GULF HAMMOCK

02314000 OTTER CREEK AT OTTER CREEK

02314200 TENMILE CREEK AT LEBANON STATION

02314205 HORSE HOLE CREEK NR LEBANON STATION

TENMILE CREEK AT MOUTH

WACCASASSA RIVER AT MOUTH

\begin{tabular}{|c|c|c|c|c|c|c|}
\hline $\begin{array}{l}\text { SITE } \\
\text { TYPE }\end{array}$ & $\begin{array}{c}\text { DRA INAGE } \\
\text { AREA } \\
\text { SQ.MI. }\end{array}$ & $\begin{array}{l}\text { DRA INAGE } \\
\text { AREA } \\
\text { QUAL IF IER }\end{array}$ & $\begin{array}{c}\text { LAT } \\
\mathrm{DG} / \mathrm{MI} / \mathrm{SO}\end{array}$ & $\begin{array}{c}\text { LONG } \\
\mathrm{DG} / \mathrm{MI} / \mathrm{SD}\end{array}$ & QUADRANGLE NAME & $\begin{array}{l}\text { CNTY } \\
\text { CODE }\end{array}$ \\
\hline SW & 220 & APPROX & $29 / 28 / 32$ & $82 / 42 / 58$ & BRONSON & 075 \\
\hline SW & 300 & APPROX & $29 / 21 / 15$ & $82 / 44 / 06$ & BRONSON SW & 075 \\
\hline SW & 480 & APPROX & $29 / 12 / 14$ & $82 / 46 / 09$ & WACCASASSA BAY & 075 \\
\hline SW & 300 & & $29 / 19 / 08$ & $82 / 47 / 03$ & OTTER CRE\&K & 075 \\
\hline SW & 26 & APPROX & $29 / 09 / 39$ & $80 / 38 / 21$ & LEBANON STATION & 075 \\
\hline SW & 8.1 & APPROX & $29 / 08 / 01$ & $82 / 38 / 14$ & LEBANON STATION & 075 \\
\hline SW & 46.0 & & $29 / 10 / 52$ & $82 / 45 / 15$ & WACCASASSA BAY & \\
\hline SW & 610 & APPROX & $28 / 10 / 02$ & $82 / 48 / 18$ & WACCASASSA BAY & \\
\hline
\end{tabular}

COASTAL AREA BETWEEN SUWANNEE AND AUCILLA RIVERS

SITE

NAME ANO LOCATION

02324000 STEINHATCHEE RIVER NR CROSS CITY

02324005 STEINHATCHEE RIVER NR HINES

STEINHATCHEE RIVER AT MOUTH

02324400 FENHOLLOWAY RIVER NR FOLEY

02324500 FENHOLLOWAY RIVER AT FOLEY

02324690 FENHOLLOWAY RIVER AT US HIGHWAY 19 NR FENHOLLOW

02324710 FENHOLLOWAY RIVER AT STATE HWY 221 NR PERRY

02324713 FENHOLLOWAY R AT COUNTRY CLUB ROAD NR PERRY

02324929 FENHOLLOWAY RIVER AB WALDO SPRING NR PERRY

02324988 FENHOLLOWAY RIVER AT HAMPTON SPRINGS

02325000 FENHOLLOWAY RIVER NR PERRY

02325200 PIMPLE CREEK AT PERRY

PIMPLE CREEK AT MOUTH

\begin{tabular}{|c|c|c|c|c|c|c|}
\hline $\begin{array}{l}\text { SITE } \\
\text { TYPE }\end{array}$ & $\begin{array}{l}\text { DRAINAGE } \\
\text { AREA } \\
\text { SQ.MI. }\end{array}$ & $\begin{array}{l}\text { DRAINAGE } \\
\text { AREA } \\
\text { QUALIF IER }\end{array}$ & $\begin{array}{c}\text { LAT } \\
\mathrm{DG} / \mathrm{MI} / \mathrm{SD}\end{array}$ & $\begin{array}{c}\text { LONG } \\
\mathrm{DG} / \mathrm{MI} / \mathrm{SO}\end{array}$ & QUADRANGLE NAME & $\begin{array}{l}\text { CNTY } \\
\text { CODE }\end{array}$ \\
\hline SW & 350 & APPROX & $29 / 47 / 11$ & $83 / 19 / 18$ & CLARA & 123 \\
\hline SW & 380 & APPROX & $29 / 46 / 27$ & $83 / 15 / 27$ & CLARA & 029 \\
\hline SW & 590 & APPROX & $29 / 40 / 06$ & $83 / 27 / 33$ & STE INHATCHEE & \\
\hline SW & 60 & APPROX & $30 / 05 / 53$ & $83 / 28 / 19$ & FENHOLLOWAY & 123 \\
\hline SW & 120 & APPROX & $30 / 03 / 55$ & $83 / 34 / 29$ & PERRY & 123 \\
\hline SW & 120 & ABOUT & $30 / 03 / 55$ & $83 / 34 / 29$ & PERRY & 123 \\
\hline SW & 120 & ABOUT & $30 / 03 / 32$ & $83 / 35 / 20$ & PERRY & 123 \\
\hline SW & 130 & ABOUT & $30 / 03 / 04$ & $83 / 36 / 35$ & PERRY & 123 \\
\hline SW & 130 & ABOUT & $30 / 02 / 58$ & $83 / 37 / 48$ & HAMPTON SPRINGS & 123 \\
\hline SW & 160 & ABOUT & $30 / 04 / 27$ & $83 / 39 / 18$ & HAMPTON SPRINGS & 123 \\
\hline SW & 160 & ABOUT & $30 / 04 / 16$ & $83 / 39 / 45$ & HAMPTON SPRINGS & 123 \\
\hline SW & 12.0 & & $30 / 07 / 38$ & $83 / 34 / 57$ & BOYD & 123 \\
\hline SW & 13.0 & & $30 / 06 / 54$ & $83 / 35 / 41$ & PERRY & \\
\hline
\end{tabular}


DRAINAGE AREAS FOR SELECTED STREAM SITES IN FLORIDA

SUWANNEE AND AUCILLA RIVERS

AUCILLA RIVER BASIN

SITE

NUMBER

NAME. AND LOCATION

AUCILLA RIVER BASIN IN GEORGIA

02326242 AUCILLA RIVER NR ASHVILLE

02326250 AUCILLA RIVER NR AUCILLA

LITTLE AUCILLA RIVER BASIN IN GEORGIA

02326261 LITTLE AUCILLA RIVER NR CHERRY LAKE

02326300 LITTLE AUCILLA RIVER NR GREENVILLE

LITTLE AUCILLA RIVER AT MOUTH

02326500 AUCILLA RIVER AT LAMONT

02326512 AUCILLA RIVER NR SCANLON

02326526 WACISSA RIVER NR WACISSA

WACISSA RIVER AT MOUTH

AUCILLA RIVER AT MOUTH

02325500 SPRING CREEK NR PERRY

SPRING CREEK AT MOUTH

FENHOLLOWAY RIVER AT MOUTH

02326000 ECONFINA RIVER NR PERRY

ECONFINA RIVER AT MOUTH $\begin{array}{lcllll} & \text { DRAINAGE } & \text { DRAINAGE } & & \\ \text { SITE } & \text { AREA } & \text { AREA } & \text { LAT LNTY } \\ \text { TYPE } & \text { SQ.MI. } & \text { LUALIFIER DG/MI/SD DG/MI/SD QNADRANGL NAME } & \text { CODE }\end{array}$

SW 217 GREENVILLE

SW $223 \quad 30 / 35 / 45 \quad 83 / 43 / 15$ ASHEVILLE

SW $345 \quad 30 / 29 / 3183 / 43 / 53$ GREENVILLE

065

SW $\quad 10.2$

SW $\quad 13.9$

SW $\quad 90.7$

SW $\quad 136$

SW $\quad 747$

SW $\quad 805$

SW 21.8

SW 98.9

SW $\quad 952.00$

SW 90.0

SW 90.0

SW $\quad 320$

SW $\quad 198$

SW $\quad 239$

\section{$30 / 29 / 3183 / 43 / 53$ GREENVILLE
GREENVILLE}

$30 / 37 / 36 \quad 83 / 29 / 44$ NANKIN

$30 / 31 / 1083 / 35 / 20$ HAMBURG

30/28/46 83/43/39 GREENVILLE

$30 / 22 / 1183 / 48 / 25$ LAMONT SE

30/13/52 83/55/08 NUTALL RISE

$30 / 18 / 04$ 83/58/47 WACISSA

30/08/52 83/58/21 NUTALLRISE

30/05/15 83/59/27 ROCK ISLANDS

$30 / 04 / 48$ 83/39/47 HAMPTON SPRINGS

$30 / 04 / 2483 / 40 / 01$ HAMPTON SPRINGS

29/58/34 83/47/08 ROCK ISLANDS

$30 / 10 / 14$ 83/49/26 JOHNSON HAMMOCK

$30 / 02 / 1283 / 55 / 40$ SNIPE ISLAND

SUWANNEE RIVER BASIN ABOVE WIIHLACOOCHEE RIVER EXCLUDING ALAPAHA RIVEK BASIN

SI TE
NUMBER

NAME AND LOCATION

SUWANNEE RIVER RASIN IN GEORGIA

SU'WANNEE RIVER ABOVE WITHL ACOOCHEE RIVER

02314986 ROCKY CREEK NR RELMONT

ROCKY CREEK AT MOUTH

02315000 SUWANNEE RIVER NR BENTON

\begin{tabular}{|c|c|c|c|c|c|c|c|}
\hline $\begin{array}{l}\text { SITE } \\
\text { TYPE }\end{array}$ & $\begin{array}{l}\text { DRAINAGE } \\
\text { AREA } \\
\text { SQ.MI. }\end{array}$ & $\begin{array}{l}\text { DRA INAGE } \\
\text { AREA } \\
\text { QUALIF IER }\end{array}$ & $\begin{array}{c}\text { LAT } \\
D G / M I / S D\end{array}$ & $\begin{array}{c}\text { LONG } \\
\mathrm{DG} / \mathrm{MI} / \mathrm{SD}\end{array}$ & QUADRANGLE & NAME & $\begin{array}{l}\text { CNTY } \\
\text { CODE }\end{array}$ \\
\hline SW & 5,720 & APPROX & & & & & \\
\hline SW & 4,610 & APPROX & $30 / 23 / 07$ & $83 / 10 / 06$ & ELLAVILLE & & \\
\hline SW & 50.00 & APPROX & $30 / 32 / 40$ & $82 / 44 / 02$ & FARGO SW & & 047 \\
\hline SW & 50.00 & APPROX & $30 / 31 / 51$ & $82 / 43 / 39$ & FARGO SW & & \\
\hline SW & $2,090.00$ & APPROX & $30 / 30 / 26$ & $82 / 42 / 59$ & FARGO SW & & 047 \\
\hline
\end{tabular}


DRAINAGE AREAS FOR SELECTED STREAM SITES IN FLORIDA

SUWANNEE AND AUCILLA RIVERS

SUWANNEE RIVER BASIN ABOVE WITHLACOOCHEE RIVER EXCLUDING ALAPAHA RIVER BASIN

SITE
NUMBER

NAME AND LOCATION

02315005 HUNTER CREEK NR BELMONT

HUNTER CREEK AT MOUTH

02315090 ROARING CREEK NR BELMONT

ROARING CREEK AT MOUTH

02315200 DEEP CREEK NR SUWANNEE VALLEY

DEEP CREEK AT MOUTH

02315392 ROBINSON CREEK NR SUWANNEE VALLEY

ROBINSON CREEK AT MOUTH

02315470 FALLING CREEK NR WINF IELD

FALLING CREEK AT SINK HOLE NR WINF IELD

FALLING CREEK AT MOUTH

02315500 SUWANNEE RIVER AT WHITE SPRINGS

02315515 SWIFT CREEK NR GENOA

02315517 OCCIDENTAL MINE DRAINAGE DITCH NR GENOA

02315518 OCCIDENTAL MINE DR D AT STATE HWY S137 NR GENOA

02315520 SWIFT CREEK AT FACIL

SWIFT CREEK AT MOUTH

02315532 ROCKY CREEK NR HOUSTON

02315534 ROCKY CREEK TRIBUTARY NR WELLBORN

ROCKY CREEK AT MOUTH

02315542 CAMP BRANCH NR GENOA

02315550 SUWANNEE RIVER AT SUWANNEE SPRINGS

02315602 SUWANNEE RIVER NR SUWANNEE

02315607 SUWANNEE RIVER NR MARION

02315615 SUWANNEE RIVER NR ADAMS

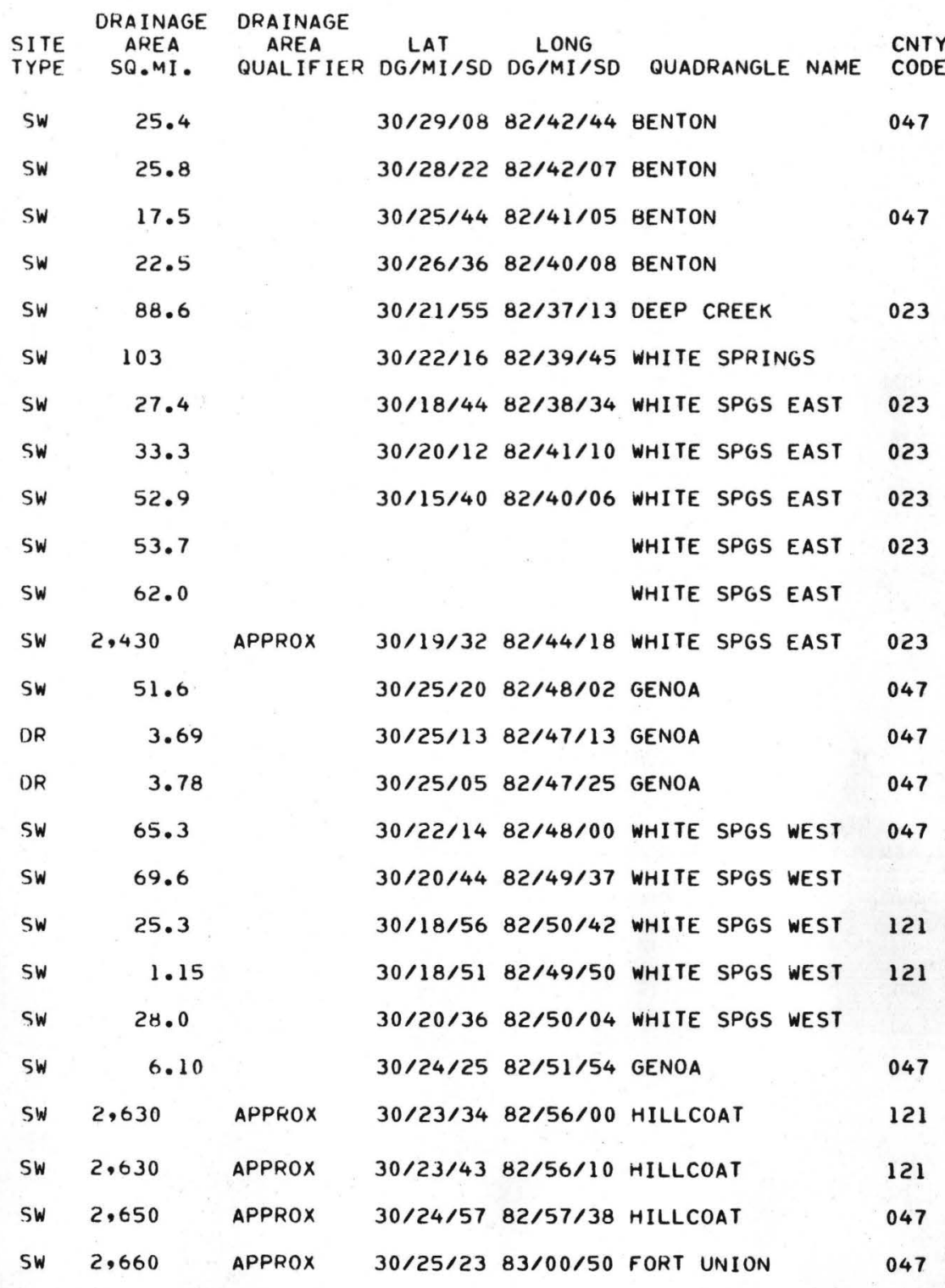


DRAINAGE AREAS FOR SELECTED STREAM SITES IN FLORIDA

SUWANNEE AND AUCILLA RIVERS

ALAPAHA RIVER BASIN

SITE
NUMBER

NAME ANO LOCATION

ALAPAHA RIVER BASIN IN GEORGIA

02317620 ALAPAHA RIVER NR JENNINGS

02317630 ALAPAHA RIVER NR JASPER

02317643 ALAPAHA RIVER AT I75 NR JASPER

ALAPAHA RIVER AT MOUTH

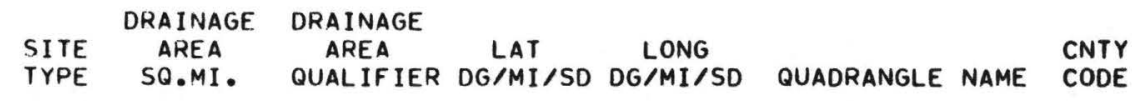

SW $1,730 \quad$ APPROX

SW 1,680 APPROX 30/35/53 83/04/24 JENNINGS 047

SW 1,720 APPROX $30 / 31 / 4283 / 02 / 17$ JENNINGS 047

SW 1,800 APPROX 30/30/00 83/02/25 FORT UNION 047

SW 1,840 APPROX 30/26/12 83/05/49 FORT UNION

WITHLACOOCHEE RIVER BASIN

SITE

NAME AND LOCATION

WITHLACOOCHEE BASIN IN GEORGIA

02319000 WITHLACOOCHEE RIVER NR PINETTA

02319300 WITHLACOOCHEE RIVER NR MADISON

02319387 NORTON CREEK AT LEE

02319390 NORTON CREEK NR LEE

NORTON CREEK AT MOUTH

WITHLACOOCHEE RIVER AT MOUTH

\begin{tabular}{|c|c|c|c|c|c|c|}
\hline $\begin{array}{l}\text { SITE } \\
\text { TYPE }\end{array}$ & $\begin{array}{c}\text { DRAINAGE } \\
\text { AREA } \\
\text { SQ.MI. }\end{array}$ & $\begin{array}{l}\text { DRAINAGE } \\
\text { AREA } \\
\text { QUALIFIER }\end{array}$ & $\begin{array}{l}\text { LAT } \\
\mathrm{DG} / \mathrm{MI} / \mathrm{SD}\end{array}$ & $\begin{array}{c}\text { LONG } \\
D G / M I / S D\end{array}$ & QUAORANGLE NAME & $\begin{array}{l}\text { CNTY } \\
\text { CODE }\end{array}$ \\
\hline SW & 2,090 & APPROX & & & ELLAVILLE & \\
\hline SW & 2,120 & APPROX & $30 / 35 / 43$ & $83 / 15 / 35$ & PINETTA & 079 \\
\hline SW & 2,240 & APPROX & $30 / 28 / 56$ & $83 / 14 / 35$ & ELLAVILLE & 047 \\
\hline SW & 55 & APPROX & $30 / 24 / 52$ & $83 / 18 / 02$ & LEE & 079 \\
\hline SW & 75 & APPROX & $30 / 24 / 51$ & $83 / 16 / 50$ & LEE & 079 \\
\hline SW & 80 & APPROX & $30 / 26 / 06$ & $83 / 13 / 18$ & ELLAVILLE & \\
\hline SW & 2,360 & APPROX & $30 / 23 / 08$ & $83 / 10 / 18$ & ELLAVILLE & \\
\hline
\end{tabular}

SUWANNEE RIVER BASIN BELOW WITHLACOOCHEE RIVER EXCLUDING SANTA FE RIVER BASIN

SITE
NUMBER

NAME AND LOCATION

02319500 SUWANNEE RIVER AT ELLAVILLE

02319508 SUWANNEE RIVER NR ELLAVILLE

02319800 SUWANNEE RIVER AT DOWLING PARK

02320000 SUWANNEE RIVER AT LURAVILLE

\begin{tabular}{|c|c|c|c|c|c|c|}
\hline $\begin{array}{l}\text { SITE } \\
\text { TYPE }\end{array}$ & $\begin{array}{c}\text { DRAINAGE } \\
\text { AREA } \\
\text { SQ.MI. }\end{array}$ & $\begin{array}{l}\text { DRAINAGE } \\
\text { AREA } \\
\text { QUAL IF IER }\end{array}$ & $\begin{array}{l}\text { LAT } \\
\mathrm{DG} / M I / S D\end{array}$ & $\begin{array}{c}\text { LONG } \\
\mathrm{DG} / \mathrm{MI} / \mathrm{SD}\end{array}$ & QUADRANGLE NAME & $\begin{array}{l}\text { CNTY } \\
\text { CODE }\end{array}$ \\
\hline SW & 6,970 & APPROX & $30 / 23 / 04$ & $83 / 10 / 19$ & ELLAVILLE & 121 \\
\hline SW & 6,980 & APPROX & $30 / 22 / 42$ & $83 / 11 / 16$ & ELLAVILLE & 121 \\
\hline SW & 7,190 & APPROX & $30 / 14 / 41$ & $83 / 14 / 59$ & DOWLING PARK & 067 \\
\hline SW & 7,330 & APPROX & $30 / 05 / 59$ & $83 / 10 / 18$ & MAYO & 121 \\
\hline
\end{tabular}




\section{DRAINAGE AREAS FOR SELECTED STREAM SITES IN FLORIDA
SUWANNEE AND AUCILLA RIVERS \\ SUWANNEE RIVER BASIN BELOW WITHLACOOCHEE RIVER EXCLUDING SANTA FE RIVER BASIN}

SITE.
NUMBER

NAME AND LOCATION

02320500 SUWANNEE RIVER AT BRANFORD

02323000 SUWANNEE RIVER NR BELL

02323500 SUWANNEE RIVER NR WILCOX

02323570 SUWANNEE RIVER NR OLD TOWN

02323585 LONG POND OUTLET NR CHIEFLAND

SUWANNEE RIVER AT MOUTH

SITE
NUMBER

NAME AND LOCATION

$\begin{array}{cclllll}\text { SITE } & \begin{array}{c}\text { DRAINAGE } \\ \text { AREA } \\ \text { SQ.MI. }\end{array} & \begin{array}{c}\text { DRAINAGE } \\ \text { AREA } \\ \text { QUALIFIER }\end{array} & \begin{array}{c}\text { LAT } \\ \text { DG/MI/SD }\end{array} & \begin{array}{c}\text { LONG } \\ \text { DG/MI/SD }\end{array} & \text { QUATY } \\ \text { SW } & 7,880.00 & \text { APPROX } & 29 / 57 / 20 & 82 / 55 / 40 & \text { BRANFORD } & 121 \\ \text { SW } & 9,390.00 & \text { APPROX } & 29 / 47 / 43 & 82 / 55 / 09 & \text { HATCHHEND } & 041 \\ \text { SW } & 9,640.00 & \text { APPROX } & 29 / 35 / 22 & 82 / 56 / 12 & \text { SUWANNEE RIVER } & 075 \\ \text { SW } & 9,760.00 & \text { APPROX } & 29 / 28 / 45 & 82 / 59 / 13 \text { MANATEE SPRINGS } & 029 \\ \text { DR } & 53.9 & & 29 / 27 / 50 & 82 / 53 / 21 \text { MANATEE SPRINGS } & 075 \\ \text { SW } & 9,950 & \text { APPROX } & 29 / 18 / 28 & 83 / 09 / 37 \text { SUWANNEE } & \end{array}$

SAIVTA FE RIVER BASIN

\begin{tabular}{|c|c|c|c|c|c|c|}
\hline $\begin{array}{l}\text { SITE } \\
\text { TYPE }\end{array}$ & $\begin{array}{l}\text { DRAINAGE } \\
\text { AREA } \\
\text { SQ.MI. }\end{array}$ & $\begin{array}{l}\text { DRA INAGE } \\
\text { AREA } \\
\text { QUAL IF IER }\end{array}$ & $\begin{array}{c}\mathrm{LAT} \\
\mathrm{DG} / \mathrm{MI} / \mathrm{SD}\end{array}$ & $\begin{array}{c}\text { LONG } \\
\mathrm{DG} / \mathrm{MI} / \mathrm{SD}\end{array}$ & QUADRANGLE NAME & $\begin{array}{l}\text { CNTY } \\
\text { CODE }\end{array}$ \\
\hline SW & 5.40 & & $29 / 51 / 49$ & $82 / 04 / 29$ & KEYSTONE HEIGHTS & 007 \\
\hline SW & 75.1 & & $29 / 50 / 21$ & $82 / 09 / 49$ & WALDO & 007 \\
\hline SW & 94.9 & & $29 / 50 / 46$ & $82 / 13 / 11$ & WALDO & 001 \\
\hline SW & 19.4 & & $29 / 56 / 10$ & $82 / 06 / 43$ & STARKE & 007 \\
\hline Sw & 59.7 & & $29 / 55 / 07$ & $82 / 12 / 39$ & SAMPSON & 007 \\
\hline Sw & 74.3 & & $29 / 51 / 36$ & $82 / 13 / 47$ & WALDO & 007 \\
\hline Sw & 178 & & $29 / 50 / 49$ & $82 / 15 / 51$ & MONTEOCHA & 001 \\
\hline SW & 7.04 & & $29 / 47 / 47$ & $82 / 17 / 20$ & MONTEOCHA & 001 \\
\hline Sw & 237 & & $29 / 52 / 43$ & $82 / 20 / 12$ & BROOKER & 005 \\
\hline SW & 22.6 & & $29 / 50 / 23$ & $82 / 22 / 36$ & ALACHUA & 001 \\
\hline SW & 2.55 & & $29 / 50 / 23$ & $82 / 22 / 48$ & ALACHUA & 001 \\
\hline SW & 28.0 & & $30 / 03 / 30$ & $82 / 08 / 20$ & RAIF ORD & 007 \\
\hline SW & 88.1 & & $30 / 04 / 00$ & $82 / 11 / 03$ & RAIF ORD & 007 \\
\hline SW & 20.6 & & $30 / 01 / 40$ & $82 / 09 / 20$ & RAIF ORD & 007 \\
\hline
\end{tabular}


SITE
NUMBER

NAME. AND LOCATION

02321000 NEW RIVER NR LAKE BUTLER

02321200 RICHARD CREEK NR LAKE BUTLER

RICHARD CREEK TRIBUTARY AT MOUTH

RICHARO CREEK AT MOUTH

02321446 FIVEMILE CREEK NR DUKES

NEW RIVER AT MOUTH

02321500 SANTA FE RIVER AT WORTHINGTON SPRINGS

02321600 OLUSTEE CREEK NR LULU

02321700 SWIFT CREEK NR LAKE BUTLER

SWIFT CREEK AT MOUTH

02321800 OLUSTEE CREEK NR PROVIDENCE

OLIJSTEE CREEK AT MOUTH

02321897 HAMMOCK BRANCH NR FORT WHITE

02321898 SANTA FE RIVER AT OLENO STATE PARK

02321961 SANTA FE RIVER NR TRAXLER

02322000 SANTA FE RIVER NR HIGH SPRINGS

02322008 TURKEY CREEK NEAR HAGUE

02322020 TURKEY CR TRIB. AT HAGUE

02322070 SANTA FE RIVER .75 MILE BL US HWY 27 NR HIGH SG 02322240 SANTA FE RIVER BL LILLY SPRING NR FORT WHITE

02322500 SANTA FE RIVER NR FORT WHITE

02322540 SANTA FE RIVER AT STATE HIGHWAY 47 NR FORT WHIT 02322590 COW CREEK NR FORT WHITE

02322660 ROSE CREEK NR COLUMBIA
DRAINAGE DRAINAGE

AREA LAT LONG CNTY $\begin{array}{llll}\text { SITE } & \text { AREA AREA } \\ \text { TYPE } & \text { SUMI. QUALIFIER DG/MI/SD DG/MI/SD QUADRANGLE NAME CODE }\end{array}$

SW $\quad 191$

SW $\quad 13.9$

SW $\quad 4.77$

SW 29.2

SW $\quad 11.8$

SW $\quad 276$

SW $\quad 575$

SW $\quad 49.1$

SW $\quad 46.0$

SW 81.9

SW $\quad 163$

SW $\quad 186$

SW 2.90

SW $\quad 820$

SW 851

SW $\quad 868$

SW $\quad 5.65$

SW $\quad 1.68$

SW 971

SW $\quad 977$

SW $\quad 1,017$

SW 1,027

SW $\quad 89$

SW $\quad 26.2$

29/59/53 82/16/27 BROOKER 125

30/01/10 82/18/59 LAKE BUTLER 125

30/00/33 82/19/50 LAKE BUTLER

29/57/41 82/20/37 BROOKER

29/58/05 82/22/49 WORTHINGTON SPGS 125

29/55/22 82/25/08 WORTHINGTON

$29 / 55 / 1882 / 25 / 35$ WORTHINGTON SPGS 001

30/05/42 82/28/25 LULU 023

30/03/28 82/25/10 LULU 125

30/01/50 82/32/04 ELLISVILLE

$30 / 00 / 1482 / 34 / 20$ ELLISVILLE

023

29/56/28 82/31/48 MIKESVILLE

29/56/07 82/36/46 MIKESVILLE

023

29/54/51 82/34/48 MIKESVILLE 001

29/52/11 82/35/21 HIGH SPRINGS 001

$29 / 50 / 3382 / 37 / 52$ HIGH SPRINGS SW 023

$29 / 44 / 4582 / 24 / 25$ GAINESVILLE WEST 001

29/46/15 82/25/47 ALACHUA 001

$29 / 49 / 5882 / 38 / 08$ HIGH SPRINGS SW 001

29/49/53 82/39/59 HIGH SPRINGS SW 023

29/50/55 82/42/55 HIGH SPRINGS SW 041

29/51/54 82/44/25 HIGH SPRINGS SW 023

ABOUT 29/51/21 85/45/24 BELL 041

30/04/23 82/40/38 COLUMBIA 023 
ORAINAGE AREAS FOR SELECTED STREAM SITES IN FLORIDA

SUWANNEE AND AUCILLA RIVERS

SANTA FE RIVER BASIN

SITE
NUMBER
NAME AND LOCATION

ICHETUCKNEE RIVER AT MOUTH

02322800 SANTA FE RIVER NR HILDRETH

02322820 SANTA FE RIVER NR 'BRANFORD

SANTA FE RIVER AT MOUTH

\begin{tabular}{|c|c|c|c|c|c|c|c|}
\hline $\begin{array}{l}\text { SITE } \\
\text { TYPE }\end{array}$ & $\begin{array}{c}\text { DRAINAGE } \\
\text { AREA } \\
\text { SQ.MI. }\end{array}$ & $\begin{array}{l}\text { DRAINAGE } \\
\text { AREA } \\
\text { QUALIFIER }\end{array}$ & $\begin{array}{c}\text { LAT } \\
D G / M I / S D\end{array}$ & $\begin{array}{c}\text { LONG } \\
\mathrm{DG} / \mathrm{MI} / \mathrm{SD}\end{array}$ & QUADRANGLE & NAME & $\begin{array}{l}\text { CNTY } \\
\text { CODE }\end{array}$ \\
\hline SW & 210 & & $29 / 55 / 56$ & $82 / 48 / 01$ & HILDRETH & & \\
\hline SW & 1,374 & & $29 / 54 / 41$ & $82 / 51 / 38$ & HILDRETH & & 041 \\
\hline SW & 1,375 & & $29 / 54 / 00$ & $82 / 52 / 00$ & HILDRETH & & 121 \\
\hline SW & 1,380 & & $29 / 53 / 13$ & $82 / 52 / 46$ & BRANF ORO & & \\
\hline
\end{tabular}

OCHLOCKONEE RIVER

ST. MARKS AND WAKULLA RIVERS AND COASTAL AREA BETWEEN AUCILLA AND OCHLOCKONEE RIVERS
SITE

NUMBER

NAME AND LOCATION

ST MARKS RIVER BASIN IN GEORGIA

02326598 CANEY CREEK NR MONTICELLO

CANEY CREEK AT MOUTH

02326601 MICCOSUKEE LAKE OUTLET NR LLOYD

02326700 LLOYD CREEK AT LLOYD

02326800 COPELAND SINK DRAIN AT LLOYD

02326900 ST MARKS RIVER NR NEWPORT

ST MARKS RIVER AT MOUTH

$\begin{array}{cc}\text { SITE } & \begin{array}{c}\text { DRAINAGE } \\ \text { AREA } \\ \text { TYPE } \\ \text { SQ.MI }\end{array} \\ \text { SW } & 114 \\ \text { SW } & 2.54 \\ \text { SW } & 5.52 \\ \text { DR } & 240 \\ \text { SW } & 31.2 \\ \text { DR } & 285 \\ \text { SW } & 535 \\ \text { SW } & 871\end{array}$

DRA INAGE

AREA LAT LONG

QUALIFIER DG/MI/SD DG/MI/SD QUADRANGLE NAME

CNTY
CODE

14

2.54

30/30/52 83/56/24 LAKE MICCOSUKEE

065

$30 / 32 / 34$ 83/57/58 LAKE MICCOSUKEE

30/31/43 83/58/52 LAKE MICCOSUKEE

065

$30 / 28 / 4184 / 00 / 31$ LLOYD

065

$30 / 28 / 4084 / 00 / 51$ LLOYO

065

30/16/00 84/09/00 WOODVILLE

129 
DRAINAGE AREAS FOR SELECTED STREAM SITES IN FLORIDA

OCHLOCKONEE RIVER

OCHLOCKONEE RIVER BASIN

SITE

NAME AND LOCATION

02327050 SOPCHOPPY RIVER NR ARRAN

02327100 SOPCHOPPY RIVER NR SOPCHOPPY

SOPCHOPPY RIVER AT MOUTH

OCHLOCKONEE RIVER BASIN IN GEORGIA

02328522 OCHLOCKONEE RIVER NR CONCORD

02328525 SHAW CREEK NR CONCORD

SHAW CREEK AT MOUTH NR CONCORD

02328859 OCHLOCKONEE RIVER TRIBUTARY NR HAVANA

02328995 LEWIS CREEK NR HAVANA

LEWIS CREEK AT MOUTH NR GIBSON

02329104 OX BOTTOM CREEK NR TALLAHASSEE

02329161 FORDS ARM TRIBUTARY AT TALLAHASSEE

02329180 MEGGINNIS ARM TRIBUTARY AT TALLAHASSEE

02329182 TRIBUTARY TO MEGGINNIS ARM TRIBUTARY AT TALLAHA

02329184 MEGGINNIS ARN TRIBUTARY AB US HIGHWAY I-10 AT T

02329186 MEGGINNIS ARM TRIBUTARY BL US HIGHWAY I-10 AT T

02329252 OCHLOCKONEE RIVER AT OCHLOCKONEE

02329256 OCHLOCKONEE RIVER TRIBUTARY AT OCHLOCKONEE

02329260 MIDWAY BRANCH NR MIDWAY

LITTLE RIVER BASIN IN GEORGIA

02329352 ATTAPULGUS CREEK AT JAMIESON

02329404 SWAMP CREEK AT JAMIESON

SWAMP CREEK AT MOUTH

\begin{tabular}{|c|c|c|}
\hline $\begin{array}{l}\text { SITE } \\
\text { TYPE }\end{array}$ & $\begin{array}{c}\text { DRAINAGE } \\
\text { AREA } \\
\text { SQ.MI. }\end{array}$ & $\begin{array}{c}\text { DRA INAG } \\
\text { AREA } \\
\text { QUAL IF I }\end{array}$ \\
\hline SW & 51.1 & \\
\hline SW & 102 & \\
\hline SW & 172 & \\
\hline SW & 1,170 & APPROX \\
\hline$S W$ & 1,002 & \\
\hline SW & 11.4 & \\
\hline SW & 11.9 & \\
\hline SW & 1.34 & \\
\hline \multirow[t]{2}{*}{ SW } & 2.65 & \\
\hline & 6.86 & \\
\hline SW & 1,140 & APPROX \\
\hline SW & 2.36 & \\
\hline SW & 1.66 & \\
\hline SW & 1.46 & 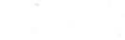 \\
\hline SW & .45 & \\
\hline SW & 3.06 & \\
\hline SW & 3.10 & \\
\hline SW & 1,220 & APPROX \\
\hline SW & .59 & \\
\hline SW & 1.33 & \\
\hline SW & 174 & \\
\hline SW & 95.6 & \\
\hline SW & 53.0 & \\
\hline SW & 57.4 & \\
\hline
\end{tabular}

LAT LONG

30/13/50 84/32/20 BRADWELL BAY

CNTY

30/07/45 84/29/40 CRAWFORDVILLE WES 129

$29 / 59 / 2584 / 25 / 18$ ST TERESA BEACH

ST TERESA BEACH

30/40/08 84/38/19 CALVARY

039

30/39/54 84/19/07 CLAVARY

$30 / 39 / 1084 / 18 / 04$ CALVARY

$30 / 39 / 2584 / 22 / 51$ HAVANA NORTH

039

$30 / 35 / 3784 / 23 / 47$ HAVANA NORTH

$30 / 34 / 3284 / 22 / 34$ HAVANA NORTH

$30 / 33 / 1484 / 23 / 03$ HAVANA SOUTH

073

30/33/02 84/18/15 LAKE JACKSON

30/29/59 84/16/39 TALLAHASSEE

073

073

$30 / 28 / 4084 / 17 / 41$ TALLAHASSEE

073

$30 / 28 / 3684 / 17 / 52$ TALLAHASSEE

073

30/29/01 84/18/01 TALLAHASSEE

073

30/29/06 84/18/06 TALLAHASSEE

073

$30 / 28 / 2584 / 24 / 25$ MIDWAY

073

$30 / 27 / 4684 / 24 / 13$ MIOWAY

073

$30 / 29 / 1984 / 25 / 30$ MIDWAY

039

$30 / 39 / 4684 / 27 / 48$ HAVANA NORTH

039

$30 / 39 / 4484 / 26 / 55$ HAVANA NORTH

039 
SITE

NUMBER

NAME AND LOCATION

ATTAPULGUS CRFEK AT CNF WITH WILLACOOCHEE CREEK

02329481 WILLACOOCHEE CREEK TRIBUTARY NR QUINCY

WILLACODCHEE CREEK TRIBUTARY AT MOUTH

02329487 WILLACHOOCHEE CREEK NR DOGTOWN

02329490 WILLACOOCHEF C NR QUINCY

02329500 LITTLE RIVER NR QUINCY

02329516 QUINCY CREEK NR QUINCY

02329532 QUINCY CREEK TRIBUTARY NR QUINCY

02329534 QUINCY C AT STATE ST HWY 267 AT QUINCY

02329538 HOLMAN BRANCH NR QUINCY

HOLMAN BRANCH AT MOUTH

02329542 QUINCY CREEK AT QUINCY

02329546 SOUTH PRONG TANYARD BRANCH NR QUINCY

02329548 TANYARD BRANCH NR QUINCY

TANYARO BRANCH AT MOUTH

02329553 HURBERT BRANCH NR OUINCY

HUBBERT BRANCH AT MOUTH

02329556 WINKLEY BRANCH NR QUINCY

WINKLEY RRANCH AT MOUTH

QUINCY CREEK AT MOUTH

02329565 LITTLE RIVER NR LITTMAN

02329582 HURRICANE CREEK NR HAVANA

02329591 HURRICANE CREEK NR QUINCY

HURRICANE CREEK AT MOUTH

02329600 LITTLE RIVER NR MIOWAY
DRAINAGE DRAINAGE

$\begin{array}{llllll}\text { SITE } & \text { AREA } & \text { AREA } & \text { LAT LONG } & & \text { CNTY } \\ \text { TYPE } & \text { SO.MI } & \text { QUALIFIER DG/MI/SD DG/MI/SD } & \text { QUADRANGLE NAME } & \text { CODE }\end{array}$

SW $\quad 164$

SW $\quad 1.26$

$1.2630 / 41 / 1884 / 32 / 00$ DOGTOWN

$30 / 40 / 5784 / 33 / 08$ DOGTOWN

$30 / 38 / 1384 / 30 / 02$ DOGTOWN

36.5

64.9

237

$30 / 35 / 1484 / 29 / 48$ HAVANA SOUTH

SW
30/36/08 84/35/20 QUINCY

$30 / 36 / 0084 / 34 / 50$ QUINCY

$30 / 36 / 3484 / 34 / 57$ QUINCY

$30 / 35 / 5684 / 34 / 34$ QUINCY

$30 / 35 / 3284 / 33 / 49$ QUINCY

30/34/08 84/34/17 QUINCY

$30 / 34 / 4284 / 33 / 30$ QUINCY

30/34/59 84/33/02 QUINCY

$30 / 35 / 3984 / 32 / 48$ QUINCY

30/35/11 84/31/36 QUINCY

30/36/03 84/32/02 QUINCY

$30 / 35 / 1784 / 01 / 36$ QUINCY

$30 / 34 / 1884 / 30 / 41$ HAVANA SOUTH

$30 / 33 / 1284 / 30 / 54$ QUINCY

$30 / 34 / 5784 / 28 / 44$ HAVANA SOUTH

$30 / 33 / 1184 / 30 / 38$ QUINCY

$30 / 33 / 0284 / 30 / 47$ QUINCY

$30 / 30 / 4484 / 31 / 25$ QUINCY
30/35/53 84/35/35 QUINCY
039

039

039

039

039

039

039

039

039

039

039

039

039

039

039

039 
SITE
NUMBER

NAME ANO LOCATION

02329625 MONROE CREEK NR MIDWAY

MONROE CREEK AT MOUTH

02329646 RICHLANDER CREEK NR QUINCY

RICHLANDER CREEK AT MOUTH

LITTLE RIVER AT MOUTH

02329668 POLK CREEK NR BLOXHAM

02329669 POLK CREEK AT MOUTH

02329700 ROCKY COMFORT CREEK NR QUINCY

02329775 ROCKY COMFORT CREEK AT WETUMPKA

02329777 ROCKY COMFORT CREEK NR WETUMKA

02329815 BEAR CREEK NR WETUMPKA

ROCKY COMFORT CREEK AT MOUTH

02329829 HARVEY CREEK NR BLOXHAM

02329835 TALQUIN, LAKE TR NR BLOXHAM

02329865 OCKLAWAHA CREEK NR GREENSBORO

02329877 OCKLAWAHA CREEK NR WETUMPKA

02329890 FREEMAN CREEK NR BLOXHAM

02329898 HAMMOCK CREEK NR WETUMPKA

02329900 LAKE TALQUIN NR BLOXHAM

02330000 OCHLOCKONEE RIVER NR BLOXHAM

02330040 TELOGIA CREEK NR GRETNA

02330045 BLUE CREEK NR HOSFORD

BLUE CREEK AT MOUTH

02330048 BLACK CREEK NR WARD

DRAINAGE DRAINAGE
AREA

$\begin{array}{lclll}\text { SITE } & \text { AREA } & \text { AREA } & \text { LAT } & \text { LONG } \\ \text { TYPE } & \text { SQ.MI. } & \text { QUALIFIER DG/MI/SO DG/MI/SO } & \text { QUADRANGLE NAME } & \text { CNTY } \\ \text { CODE }\end{array}$

$\begin{array}{lll}\text { TYPE } & \\ \text { SW } & 6.80 & 30 / 30 / 3384 / 29 / 54 \text { HAVANA SOUTH }\end{array}$

SW $\quad 8.20$

SW $\quad 5.80$

SW 9.66

SW $\quad 329$

SW $\quad 3.64$

SW $\quad 4.84$

SW $\quad 9.46$

SW 31.1

SW 34.1

SW $\quad 4.78$

SW $\quad 10.20$

SW $\quad 56.3$

SW 8.11

SW .48

SW 11.1

SW 28.8

SW $\quad 7.95$

SW 6.82

RE 1,690

SW 1,700

SW $\quad 6.42$

SW $\quad 11.6$

SW 13.3

SW 18.2
$30 / 30 / 1984 / 03 / 19$ QUINCY

$30 / 31 / 18$ 84/33/15 QUINCY

$30 / 29 / 0384 / 31 / 59$ QUINCY

$30 / 28 / 4984 / 32 / 13$ LAKE TALQUIN

$30 / 25 / 4984 / 31 / 47$ LAKE TALQUIN

30/26/05 84/32/17 LAKE TALQUIN

$30 / 32 / 4484 / 38 / 09$ GRETNA

$30 / 29 / 38$ 84/36/55 LAKE TALQUIN

30/29/05 84/35/35 LAKE TALQUIN

30/29/10 84/35/33 LAKE TALQUIN

$30 / 28 / 1484 / 35 / 30$ LAKE TALQUIN

$30 / 27 / 50$ 84/33/42 LAKE TALQUIN

30/24/50 84/33/05 LAKE TALQUIN

$30 / 24 / 18$ 84/23/49 LAKE TALQUIN

$30 / 27 / 4584 / 44 / 20$ BLOXHAM

$30 / 27 / 0084 / 38 / 36$ BLOXHAM

30/24/03 84/35/36 LAKE TALQUIN

30/25/27 84/38/54 BLOXHAM

30/23/15 84/38/35 BLOXHAM

30/22/59 84/39/18 BLOXHAM

30/37/32 84/41/20 GRETNA

$30 / 21 / 1484 / 43 / 18$ WARO

$30 / 20 / 0284 / 42 / 44$ WARD

$30 / 19 / 3284 / 41 / 37$ WARD
073

039

073

039

039

039

039

039

073

039

039

073

039

073

077

039

077

073 
DRAINAGE AREAS FOR SELECTEO STREAM SITES IN FLORIDA OCHLOCKONEE RIVER

OCHLOCKONEE RIVER BASIN

SITE

NUMBER

NAME ANI LOCATION

BLACK CREEK AT MOUTH

02330049 SWEETWATER CRFEK NR WARD

SWEETWATER CREEK. AT MOUTH

02330050 TELOGIA CREEK NR GREENSBORO

02330065 JUNIPER CREEK NR GREENSBORO

JUNIPER CREEK AT MOUTH

02330080 MULE CREEK NF GREENSBORO

MULE CREEK AT MOUTH

02330100 TELOGIA CREEK NR BRISTOL

02330102 MILL BRANCH NR ARISTOL

MILL BRANCH AT MOUTH

02330120 TELOGIA CREEK AT HOSFORD

02330122 BIG CREEK NR HOSFORD

02330129 YELLOW CREEK NR WARD

YELLOW CREEK AT MOUTH

TELOGIA CREEK AT MOUTH

02330145 INDIAN CREEK NR SMITH CREEK

02330150 OCHLOCKONEE RIVER NR SMITH CREEK

02330155 TIGER CREEK NR SMITH CREEK

02330166 OCHLOCKONEE RIVER NR MCINTYRE

OCHLOCKONEE RIVER AT MOUTH
ORAINAGE DRAINAGE

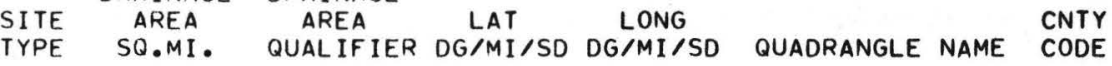

SW $18.4 \quad 30 / 19 / 13 \quad 84 / 42 / 08$ WARD

SW $2.59 \quad 30 / 18 / 2584 / 42 / 01$ WARD

SW $2.83 \quad 30 / 18 / 3984 / 42 / 26$ WARD

SW 28.1

SW $\quad 8.50$

$30 / 33 / 3484 / 43 / 36$ GRETNA

$30 / 31 / 4984 / 44 / 16$ GRETNA

$30 / 31 / 5784 / 44 / 39$ GRETNA

$30 / 30 / 40 \quad 84 / 49 / 41$ SYCAMORE

$30 / 30 / 1584 / 49 / 10$ SYCAMORE

$30 / 25 / 3584 / 55 / 40$ BRISTOL

30/25/45 84/56/08 BRISTOL

30/25/29 84/55/52 BRISTOL

$30 / 22 / 2584 / 48 / 22$ TELOGIA

$30 / 23 / 20 \quad 84 / 47 / 24$ HOSFORD

30/16/58 84/45/08 TELOGIA

$30 / 17 / 18 \quad 84 / 44 / 47$ WARD

$30 / 16 / 0584 / 44 / 03$ WARD

30/11/54 84/43/42 SMITH CREEK

077

SW $\quad 7.93$

30/10/35 84/40/05 SMITH CREEK

129

SW $2,080 \quad$ APPROX

30/09/46 84/42/55 SMITH CREEK

077

SW $2,230 \quad 29 / 59 / 19 \quad 84 / 30 / 09$ MCINTYRE

037

SW 2,250 
SITE
NUMBER

NAME. AND LOCATION

02357500 LAKE SEMINOLF AT CHATTAHOOCHEE

02358000 APALACHICOLA RIVER AT CHATTAHOOCHEE 02358500 NORTH MOSQUITO CREEK AT CHATTAHOOCHEE

02358519 MOSQUITO CREEK AT CHATTAHOOCHEE.

MOSQUITO CREEK AT MOUTH

02358600 FLAT CHEEK NR CHATTAHOOCHEE

FLAT CREEK AT MOUTH

02358620 CROOKED CREEK NR GREENSBORO

CROOKED CREEK AT MOUTH

02358685 LITTLE SWEETWATFR CREEK NR BRISTOL

LITTLE SWEETWATER CREEK AT MOUTH

02358700 APALACHICOLA RIVER NR RLOUNTSTOWN 02358754 APALACHICOLA RIVER NR WEWAHITCHKA 02359170 APALACHICOLA RIVER NR SUMATRA 02359228 JACKSON RIVER INR APALACHICOLA

02359230 APALACHICOLA RIVER NR APALACHICOLA APALACHICOLA AT MOUTH

SITF NAME AND LOCATION

02358800 CHIPOLA RIVER AT OAKDALE

02358998 HOLLIMAN RRANCH NR ALTHA HOLLIMAN BRANCH AT MOUTH

02359000 CHIPOLA RIVER NR ALTHA

02359101 UEAD LAKE OUTLET AT WEWAHITCHKA

CHIPOLA RIVER AT MOUTH

\begin{tabular}{|c|c|c|c|c|c|c|}
\hline $\begin{array}{l}\text { SITE } \\
\text { TYPE }\end{array}$ & $\begin{array}{l}\text { DRAINAGE } \\
\text { AREA } \\
\text { SQ.MI. }\end{array}$ & $\begin{array}{l}\text { DRAINAGE } \\
\text { AREA } \\
\text { QUALIF IER }\end{array}$ & $\begin{array}{c}\text { LAT } \\
D G / M I / S D\end{array}$ & $\begin{array}{c}\text { LONG } \\
\mathrm{DG} / \mathrm{MI} / \mathrm{SD}\end{array}$ & QUADRANGLE NAME & $\begin{array}{l}\text { CNTY } \\
\text { CODE }\end{array}$ \\
\hline RE & 17,00 & APPROX & $30 / 42 / 29$ & $84 / 51 / 56$ & CHATTAHOOCHEE & 063 \\
\hline SW & 17,200 & APPROX & $30 / 42 / 03$ & $84 / 51 / 33$ & CHATTAHOOCHEE & 063 \\
\hline SW & 57.9 & & $30 / 42 / 08$ & $84 / 49 / 35$ & CHATTAHOOCHEE & 039 \\
\hline SW & 86.2 & & $30 / 41 / 19$ & $84 / 50 / 30$ & CHATTAHOOCHEE & 039 \\
\hline SW & $89 . ?$ & & $30 / 41 / 34$ & $84 / 51 / 37$ & CHATTAHOOCHEE & \\
\hline SW & 24.9 & & $30 / 37 / 43$ & $84 / 50 / 06$ & & 039 \\
\hline SW & 51.5 & & & & SNEADS & \\
\hline SW & 11.1 & & $30 / 35 / 30$ & $84 / 52 / 54$ & TATES HELL SWAMP & 039 \\
\hline SW & 18.4 & & $30 / 38 / 04$ & $84 / 52 / 54$ & TATES HELL SWAMP & \\
\hline SW & 2.45 & & $30 / 28 / 27$ & $84 / 58 / 49$ & BRISTOL & 077 \\
\hline SW & 2.77 & & $0 / 00 / 00$ & $0 / 00 / 00$ & BRISTOL & 077 \\
\hline SW & 17,600 & APPROX & $30 / 25 / 30$ & $85 / 01 / 53$ & BLOUNTSTOWN & 013 \\
\hline SW & 17,800 & APPROX & $30 / 07 / 41$ & $85 / 08 / 42$ & DEAD LAKE & 045 \\
\hline SW & 19,200 & APPROX & $29 / 56 / 57$ & $85 / 00 / 56$ & FORBES ISLAND & 037 \\
\hline SW & 239 & & $29 / 47 / 08$ & $85 / 03 / 36$ & JACKSON RIVER & 037 \\
\hline SW & 19,500 & APPROX & $29 / 45 / 56$ & $85 / 01 / 57$ & JACKSON RIVER & 037 \\
\hline SW & 19,600 & & $29 / 43 / 30$ & $84 / 58 / 35$ & APALACHICOLA & \\
\hline
\end{tabular}

CHIPOLA RIVER BASIN

\begin{tabular}{|c|c|}
\hline $\begin{array}{l}\text { SITE } \\
\text { TYPE }\end{array}$ & $\begin{array}{c}\text { DRAINAGE } \\
\text { AREA } \\
\text { SQ.MI. }\end{array}$ \\
\hline SW & 519 \\
\hline SW & 2.04 \\
\hline SW & 2.31 \\
\hline SW & 781 \\
\hline SW & 1,205 \\
\hline SW & 1,237 \\
\hline
\end{tabular}

\section{DRAINAGE}

AREA LAT LONG
QUALIFIER DG/MI/SD DG/MI/SD QUADRANGLE NAME
30/43/02 85/12/01 OAKDALE

30/32/43 85/09/33 ALTHA WEST

30/32/46 85/10/05 ALTHA WEST

30/32/02 85/09/55 ALTHA WEST

$30 / 07 / 3785 / 10 / 38$ DEAD LAKE

045

CNTY
CODE

063

30/00/41 85/05/29 KENNEOY CREEK 
SITE NUMBER

NAME AND LOCATION

02330200 NEW RIVER AT VILAS 02330300 NEW RIVER NR WILMA

02330400 NEW RIVER NR SUMATRA

NEW RIVER AT MOUTH

$\begin{array}{cclll}\text { SITE } & \begin{array}{c}\text { DRAINAGE } \\ \text { AREA } \\ \text { SQ.MI. }\end{array} & \begin{array}{c}\text { DRAINAGE } \\ \text { AREA } \\ \text { QUALIFIER }\end{array} & \begin{array}{c}\text { LAT } \\ \text { DG/MI/SO DG/MI/SD }\end{array} & \text { LUADRANGLE NAME } \\ \text { SW } & 23.21 & 30 / 13 / 0884 / 53 / 28 \text { WILMA } & \begin{array}{c}\text { CNTY } \\ \text { CODE }\end{array} \\ \text { SW } & 81.7 & 30 / 07 / 40 & 07 / 53 / 45 \text { WILMA } & 077 \\ \text { SW } & 157 & 30 / 02 / 1984 / 50 / 38 \text { OWENS BRIDGE } & 077 \\ \text { SW } & 320 & \text { APPROX } & 29 / 51 / 5684 / 40 / 52 \text { CARRABELLE } & \end{array}$

CHOCTAWHATCHEE, YELLOW, AND ESCAMBIA RIVERS

ST. ANDREW HAY, INFLOW AND COASTAL AREA
SITE

NUMBER

NAME AND LOCATION

02359285 WETAPPO CREEK NR WEWAHITCHKA

02359300 SANDY CREEK NR PANAMA CITY

SANDY CREEK AT MOUTH

02359350 ECONFINA CREEK NR COMPASS LAKE

02359425 ECONF INA CREEK NR GREENHEAD

02359450 ECONFINA CREEK NR FOUNTAIN

02359500 ECONFINA CREEK NR BENNETT

02359550 BEAR CREEK NR YOUNGSTOWN

02359600 LITTLE BEAR CREEK AT YOUNGSTOWN

LITTLE BEAR CREEK AT MOUTH

BEAR CREEK AT MOUTH

02359610 WHITE OAK CREEK NR GREENHEAD

02359625 STILL POND NR GREENHEAD

02359650 BIG CEDAR CREEK NR BENINETT

02359660 DEER POINT LAKE NR PANAMA CITY ECONFINA CREEK AT MOUTH
DRAINAGE
AREA SQ.MI.

\section{DRAINAGE
AREA}

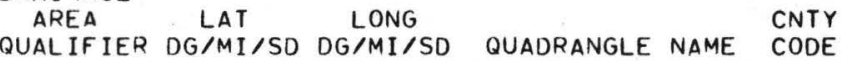
TYPE SW $\quad 45$

SW $\quad 25$

APPROX

30/03/00 85/18/20 WETAPPO CREEK

045

APPROX 30/08/27 85/24/26 NORTH OF ALLANTON 005 30/03/35 85/26/20 ALLANTON

30/33/20 85/26/05 COMPASS LAKE

$30 / 30 / 40$ 85/30/19 GAP LAKE

30/28/55 85/31/30 BENNETT

SW $\quad 122$

SW $\quad 67.2$

SW $\quad 27.4$

SW $\quad 33.9$

SW $\quad 128$

SW $\quad 8.46$

30/23/04 85/33/24 BENNETT.

005

$30 / 19 / 1085 / 27 / 20$ YOUNGSTOWN

005

$30 / 32 / 00 \quad 85 / 26 / 40$ YOUNGSTOWN

$30 / 19 / 28$ 85/27/58 YOUNGSTOWN

30/18/32 85/35/16 BAYHEAD

$30 / 33 / 1085 / 32 / 30$ GAP LAKE

30/29/45 85/34/35 BENNETT

$30 / 22 / 1085 / 37 / 20$ BAYHEAD

005

$30 / 17 / 57$ 85/34/50 BAYHEAD

005 
DRAINAGE AREAS FOR SELECTEO STREAM SITES IN FLORIDA

CHOCTAWHATCHEE, YELLOW, AND ESCAMBIA RIVERS

CHOC.TAWHATCHEE BAY, INFLOW AND COASTAL AREA

SITE
NUMBER

NAME ANI) LOCATION

02366900 MAGNOLIA CREEK NR FREEPORT

MAGNOLIA CREEK AT MOUTH

02366911 LAFAYETTE CREEK AT FREEPORT

LAFAYETTE CREEK AT MOUTH

FOURMILE AT MOUTH

02367000 ALAQUA CREEK NR DE FUNIAK SPRINGS

ALAQUA CREEK AT MOUTH

02367165 BASIN CREEK NR PORTLAND

BASIN CREEK AT MOUTH

02367240 ROCKY CREEK NR PORTLAND

02367242 LITTLE ROCKY CREEK NR NICEVILLE

LITTLE ROCKY CREEK AT MOUTH

02367250 ROCKY CREEK NR NICFVILLE

02367264 LONG CREEK NR PORTLAND

LONG CREEK AT MOUTH

02367276 LONG CREEK NO 2 NR VALPARAISO

LONG CREEK NO 2 AT MOUTH

ROCKY CREEK $\triangle T$ MOUTH

02367284 TURKEY CREEK NR VALPARAISO

TURKEY CREEK AT MOUTH

02367300 SWIFT CREEK NR NICEVILLE

SWIFT CREEK AT MOUTH

02367305 TURKEY CREEK NK NICEVILLE

ROGUE CREEK AT MOUTH

02367308 TENMILE CREEK NR VALPARAISO
DRAINAGE DRAINAGE

SITE AREA AREA LAT LONG CNTY

TYPE SQ.MI. QUALIFIER DG/MI/SD DG/MI/SD QUADRANGLE NAME CODE

SW $11.2 \quad 30 / 31 / 48 \quad 86 / 05 / 15$ ROCK HILL

131

SW $\quad 11.4$

SW $\quad 35.4$

SW $\quad 36.6$

SW $\quad 52.5$

SW $\quad 65.6$

SW $\quad 124$

SW $\quad 36.2$

SW $\quad 38.8$

SW $\quad 42.4$

SW $\quad 3.85$

SW $\quad 19.1$

SW $\quad 67.6$

SW $\quad 6.31$

SW $\quad 6.51$

SW $\quad 4.42$

SW $\quad 5.25$

SW $\quad 84.9$

SW 2.97

SW $\quad 3.11$

SW $\quad 5.96$

SW 9.37

SW 22.7

SW $\quad 6.78$

SW $\quad 16.8$

30/29/35 86/07/33 FREEPORT

30/29/10 86/08/14 FREEPORT

$30 / 28 / 2486 / 08 / 16$ FREEPORT

30/37/00 86/09/50 PORTLAND

30/29/39 86/11/55 FREEPORT

30/31/02 86/14/09 PORTLAND

30/29/58 86/14/39 PORTLAND

30/34/23 86/22/01 NICEVILLE

$30 / 36 / 3486 / 25 / 31$ NICEVILLE

30/32/41 86/22/44 NICEVILLE

$30 / 32 / 0786 / 22 / 55$ NICEVILLE

30/30/45 86/23/24 NICEVILLE

$30 / 30 / 5286 / 23 / 52$ NICEVILLE

$30 / 31 / 2086 / 24 / 42$ NICEVILLE

$30 / 30 / 2786 / 24 / 50$ NICEVILLE

30/30/27 86/25/05 NICEVILLE

$30 / 30 / 4386 / 25 / 30$ NICEVILLE

30/30/28 86/25/26 NICEVILLE

$30 / 31 / 4086 / 28 / 00$ NICEVILLE

30/30/40 86/27/09 NICEVILLE

$30 / 33 / 4386 / 32 / 10$ VALPARAISO

30/33/28 86/32/11 VALPARAISO

$30 / 34 / 1286 / 31 / 00$ VALPARAISO
091

091

091

091

091 
DRAINAGE AREAS FOR SELECTED STREAM SITES IN FLORIDA

CHOCTAWHATCHEE, YELLOW, AND ESCAMBIA RIVERS

CHOCTAWHATCHEE BAY, INFLOW AND COASTAL AREA

SITE

NAME AND LOCATION

TENMILE CREEK AT MOUTH

02367309 ANDERSON BRANCH NR VALPARAISO

02367310 JUNIPER CREEK AT STATE HIGHWAY 85 NR NICEVILLE

JUNIPER CREEK AT MOUTH

02367355 TURKEY CREEK AT US GOVT RAILROAD NR NICEVILLE

TURKEY CREEK AT MOUTH

TOMS CREEK AT MOUTH

02367370 GARNIER CREEK AT LONGWOOD

GARNIER CREEK AT MOUTH

02367377 LIGHTWOOD KNOT CREEK AT LONGWOOD

\begin{tabular}{|c|c|c|}
\hline $\begin{array}{l}\text { SITE } \\
\text { TYPE }\end{array}$ & $\begin{array}{c}\text { DRAINAGE } \\
\text { AREA } \\
\text { SQ.MI. }\end{array}$ & $\begin{array}{l}\text { DRA INAGE } \\
\text { AREA } \\
\text { QUAL IF IER }\end{array}$ \\
\hline SW & 16.9 & \\
\hline SW & 1.15 & \\
\hline SW & 27.6 & \\
\hline SW & 28.5 & \\
\hline SW & 60.8 & \\
\hline SW & 65.7 & \\
\hline SW & 8.17 & \\
\hline SW & 9.42 & \\
\hline SW & 9.72 & \\
\hline SW & 11.8 & \\
\hline SW & 11.9 & \\
\hline
\end{tabular}

NAME AND LOCATION

NUMBER

02367900 YELLOW RIVER NR OAK GROVE
02367950 YELLOW RIVER NR CANNON TOWN

02368000 YELLOW RIVER AT MILLIGAN

02368300 BAGGETT CREEK NR MILLIGAN

02368450 GUM CREEK NR DE FUNIAK SPRINGS

02368500 SHOAL RIVER NR MOSSY HEAD

02368800 POND CREEK NR DORCAS

02368817 PINE LOG CREEK NR FLOWERSVILLE

PINE LOG CREEK AT MOUTH

02368836 LONG CREEK NR MOSSY HEAD
YELLOW RIVER EASIN

\begin{tabular}{|c|c|c|c|c|c|c|}
\hline $\begin{array}{l}\text { SITE. } \\
\text { TYPE }\end{array}$ & $\begin{array}{c}\text { DRA INAGE } \\
\text { AREA } \\
\text { SQ.MI. }\end{array}$ & $\begin{array}{l}\text { DRA INAGE } \\
\text { AREA } \\
\text { QUAL IF IER }\end{array}$ & $\begin{array}{c}\mathrm{LAT} \\
\mathrm{DG} / \mathrm{MI} / \mathrm{SO}\end{array}$ & $\begin{array}{c}\text { LONG } \\
\mathrm{DG} / \mathrm{MI} / \mathrm{SD}\end{array}$ & QUADRANGLE NAME & $\begin{array}{l}\text { CNTY } \\
\text { CODE }\end{array}$ \\
\hline SW & 510 & & $30 / 55 / 30$ & $86 / 33 / 34$ & OAK GROVE & 091 \\
\hline SW & 578 & & $30 / 50 / 00$ & $86 / 36 / 00$ & CRESTVIEW NORTH & 091 \\
\hline SW & 624 & & $30 / 45 / 10$ & $86 / 37 / 45$ & BAKER & 091 \\
\hline SW & 7.77 & & $30 / 43 / 40$ & $86 / 39 / 35$ & HOLT & 091 \\
\hline SW & 24.4 & & $30 / 48 / 08$ & $86 / 11 / 42$ & LIBERTY & 131 \\
\hline Sw & 123 & & $30 / 47 / 45$ & $86 / 18 / 25$ & NEW HARMONY & 131 \\
\hline SW & 94.8 & & $30 / 50 / 02$ & $86 / 25 / 43$ & DORCAS & 091 \\
\hline SW & 22.2 & & $30 / 52 / 43$ & $86 / 21 / 42$ & PAXTON & 131 \\
\hline
\end{tabular}

$30 / 48 / 2786 / 24 / 19$ DORCAS

$30 / 49 / 4286 / 20 / 31$ NEW HARMONY
CNTY 30/34/03 86/31/06 VALPARAISO 091 $30 / 33 / 3586 / 30 / 50$ VALPARAISO 091 $30 / 33 / 2686 / 31 / 10$ VALPARAISO 091 $30 / 32 / 4386 / 31 / 24$ VALPARAISO $30 / 32 / 3586 / 31 / 15$ VALPARAISO $30 / 31 / 1586 / 29 / 53$ VALPARAISO $30 / 30 / 0686 / 30 / 12$ VALPARAISO $30 / 28 / 4986 / 35 / 07$ FORT WALTON BEACH 091 FORT WALTON BEACH 091 30/28/41 86/35/46 FORT WALTON BEACH 091 $30 / 28 / 3186 / 35 / 39$ FORT WALTON BEACH 091 
DRAINAGE AREAS FOR SELECTED STREAM SITES IN FLORIDA CHOCTAWHATCHEE, YELLOW, AND ESCAMBIA RIVERS

SITE

NUMBER NAME ANO LOCATION

LONG CREEK AT MOUTH

02368850 SHOAL RIVER AT DORCAS

02368900 SHOAL RIVER AT US HIGHWAY 90 NR CRESTVIEN

02368944 TITI CREEK NR MOSSY HEAD

02368990 TITI CKEEK NR CRESTVIEW

TITI CREEK AT MOUTH

02369000 SHOAL RIVER NR CRESTVIEW

SHOAL RIVER AT MOUTH

02369400 YELLOW RIVER NR GALLIVER

02369500 YELLOW RIVER NR HOLT

02369600 YELLOW RIVER NR MILTON

YELLOW RIVER AT MOUTH

POND CREEK AT MOUTH

SITE

NAME AND LOCATION

02369920 BLACKWATER RIVER NR GOOD HOPE

02369930 BLACKWATER RIVER NR BLACKMAN

02370000 BLACKWATER RIVER NR BAKER

02370015 MUDDY BRANCH NR BEAVER CREEK

MUDDY BRANCH $\triangle T$ MOUTH

02370100 BLACKWATER RIVER NR HOLT

02370200 BIG JUNIPER CREEK NR MUNSON
YELLOW RIVER BASIN

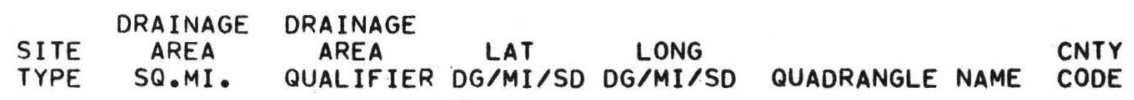

SW $29.2 \quad 30 / 48 / 03 \quad 86 / 23 / 55$ DORCAS

SW $319 \quad 30 / 47 / 27 \quad 86 / 25 / 14$ DORCAS

SW $\quad 365$

SW $\quad 15.4$

SW $\quad 62.9$

SW $\quad 78.5$

SW $\quad 474$

SW 499.

SW 1,155 .

SW 1,210

SW 1,340

SW 1,365

SW $\quad 88.0$

30/45/10 86/30/33 CRESTVIEW NORTH 091 30/42/07 85/23/16 SPENCER FLATS 131 $30 / 42 / 0586 / 29 / 28$ SPENCER FLATS 30/42/07 86/32/06 CRESTVIEW SOUTH $30 / 41 / 5086 / 34 / 15$ CRESTVIEW SOUTH 091 $30 / 41 / 1186 / 38 / 52$ HOLT $30 / 41 / 1286 / 40 / 19$ HOLT $30 / 40 / 2586 / 44 / 50$ HOLT 091 091 $30 / 34 / 1086 / 55 / 25$ WARD BASIN $30 / 33 / 2886 / 59 / 43$ MILTON SOUTH 30/36/23 87/01/53 PACE
BLACKWATER RIVER BASIN

\begin{tabular}{|c|c|c|c|c|c|c|c|}
\hline $\begin{array}{l}\text { SITE } \\
\text { TYPE }\end{array}$ & $\begin{array}{c}\text { DRAINAGE } \\
\text { AREA } \\
\text { SQ.MI. }\end{array}$ & $\begin{array}{l}\text { DRA INAGE } \\
\text { AREA } \\
\text { QUALIFIER }\end{array}$ & $\begin{array}{c}\text { LAT } \\
D G / M I / S D\end{array}$ & $\begin{array}{c}\text { LONG } \\
\mathrm{DG} / \mathrm{MI} / \mathrm{SD}\end{array}$ & QUADRANGLE & NAME & $\begin{array}{l}\text { CNTY } \\
\text { CODE }\end{array}$ \\
\hline SW & 90 & APPROX & $30 / 59 / 20$ & $86 / 43 / 12$ & BLACKMAN & & 091 \\
\hline SW & 144 & & $30 / 56 / 00$ & $86 / 44 / 09$ & BLACKMAN & & 091 \\
\hline SW & 205 & & $30 / 50 / 00$ & $86 / 44 / 05$ & BAKER & & 091 \\
\hline SW & 1.45 & & $30 / 51 / 01$ & $86 / 46 / 54$ & MUNSON & & 091 \\
\hline SW & 1.61 & & $30 / 50 / 36$ & $86 / 46 / 50$ & MUNSON & & \\
\hline SW & 276 & & $30 / 43 / 26$ & $86 / 47 / 34$ & FLORIDALE & & 113 \\
\hline $\mathbf{w}$ & 36.0 & & $30 / 51 / 50$ & $86 / 54 / 20$ & SPRING HILL & & 113 \\
\hline
\end{tabular}


DRAINAGE AREAS FOR SELECTED STREAM SITES IN FLORIDA

CHOCTAWHATCHEE, YELLOW, AND ESCAMBIA RIVERS

BLACKWATER RIVER BASIN

SITE
NUMBER

NAME AND LOCATION

02370230 SWEETWATER CREEK NR MUNSON

02370270 BIG JUNIPER CREEK NR HAROLD

02370280 EAST FORK BIG COLDWATER CREEK NR MUNSON

02370300 WEST FORK COLDWATER CREEK AT COBBTOWN

02370500 BIG COLDWATER CREEK NR MILTON

BIG COLOWATER CREEK AT MOUTH

02370700 POND CREEK NR MILTON

02370750 HURRICANE BRANCH NR MILTON

HURRICANE BRANCH AT MOUTH

BLACKWATER RIVER AT MOUTH

\begin{tabular}{|c|c|c|c|c|c|c|c|}
\hline $\begin{array}{l}\text { SITE } \\
\text { TYPE }\end{array}$ & $\begin{array}{c}\text { DRAINAGE } \\
\text { AREA } \\
\text { SQ.MI. }\end{array}$ & $\begin{array}{l}\text { DRAINAGE } \\
\text { AREA } \\
\text { QUALIF IER }\end{array}$ & $\begin{array}{c}\text { LAT } \\
D G / M I / S D\end{array}$ & $\begin{array}{c}\text { LONG } \\
\mathrm{DG} / \mathrm{MI} / \mathrm{SD}\end{array}$ & QUADRANGLE & NAME & $\begin{array}{l}\text { CNTY } \\
\text { CODE }\end{array}$ \\
\hline SW & 45.0 & & $30 / 51 / 20$ & $86 / 51 / 06$ & MUNSON & & 113 \\
\hline SW & 142 & & $30 / 43 / 46$ & $86 / 53 / 58$ & HAROLD & & 113 \\
\hline SW & 64.0 & & $30 / 52 / 56$ & $86 / 57 / 28$ & MCLELLAN & & 113 \\
\hline SW & 39.5 & & $30 / 53 / 00$ & $87 / 06 / 30$ & ALLENTON & & 113 \\
\hline SW & 237 & & $30 / 42 / 30$ & $86 / 58 / 20$ & HAROLD & & 113 \\
\hline SW & 241 & & $30 / 40 / 23$ & $86 / 57 / 42$ & HAROLD & & \\
\hline SW & 58.7 & & $30 / 40 / 50$ & $87 / 07 / 55$ & WALLACE & & 113 \\
\hline $\mathrm{SW}$ & 2.95 & & $30 / 40 / 32$ & $87 / 08 / 17$ & WALLACE & & 113 \\
\hline SW & 3.18 & & $30 / 40 / 28$ & $87 / 07 / 45$ & WALLACE & & \\
\hline SW & 860 & & $30 / 35 / 36$ & $87 / 00 / 56$ & PACE & & \\
\hline
\end{tabular}

ESCAMBIA BAY, INFLOW ANO COASTAL AREA

SITE

NUMBER NAME AND LOCATION

02367390 TURTLE CREEK NR OCEAN CITY

02367397 LIVE OAK CREEK NR FLOROSA

02367400 EAST BAY RIVER NR WYNNEHAVEN

EAST BAY RIVER AT MOUTH

02376079 CARPENTER CREEK AT PENSACOLA

BAYOU TEXAR AT MOUTH

02376084 GARDEN STREET STORM DRAIN AT PENSACOLA

02376085 TRIBUTARY TO RAYOU CHICO AT W ST AT PENSACOLA

02376087 TRIB TO BAYOU CHICO AT PENSACOLA

02376100 BAYOU MARCUS CREEK NR PENSACOLA

BAYOU MARCUS CREEK AT MOUTH
DRAINAGE DRAINAGE

$\begin{array}{lllll}\text { SITE } & \text { AREA } & \text { AREA } & \text { LAT } & \text { LONG } \\ \text { TYPE } & \text { SQ.MI. } & \text { QUALIFIER DG/MI/SO DG/MI/SD QUADRANGLE NAME }\end{array}$

$22.3 \quad 30 / 30 / 3186 / 40 / 13$ HOLT SW

$30 / 30 / 3986 / 42 / 53$ HOLT SW

$30 / 25 / 5386 / 46 / 20$ NAVARRE

$30 / 26 / 26 \quad 80 / 52 / 35$ HOLLEY

$30 / 28 / 1587 / 12 / 48$ PENSACOLA

033

$30 / 27 / 3987 / 12 / 40$ PENSACOLA

30/24/53 87/14/59 PENSACOLA

$30 / 24 / 5587 / 15 / 01$ WEST PENSACOLA

033

.54

$30 / 24 / 4287 / 16 / 45$ WEST PENSACOLA

033

1.70

$30 / 26 / 5387 / 17 / 26$ WEST PENSACOLA

033

11.2

$30 / 25 / 4887 / 20 / 29$ WEST PENSACOLA 
DRAINAGE AREAS FOR SELECTED STREAM SITES IN FLORIDA

CHOCTAWHATCHEE, YELLOW, AND ESCAMBIA RIVERS

CHOCTAWHATCHEE RIVER BELOW PEA RIVER

SITE

NAME AND LOCATION

WRIGHTS CREEK AT MOUTH

02365500 CHOCTAWHATCHEE RIVER AT CARYVILLE

02365658 SANDY CREEK NR ARGYLE

02365700 SANDY CREEK AT PONCE DE LEON

BLUE CREEK AT MOUTH

MILL CREEK AT MOUTH

SANDY CREEK AT MOUTH

02365726 BRUCE CREEK NR DEFUNIAK SPRINGS

02365758 BRUCE CREEK NR RED BAY

BRUCE CREEK AT MOUTH

SEVEN RUNS AT MOUTH

02366000 HOLMES CREEK AT VERNON

02366164 REFDY BRANCH AT NEW HOPE

REF.OY BRANCH AT MOUTH

02366500 CHOCTAWHATCHEE RIVER NR BRUCE

02366650 PINE LOG CREEK NR EBRO

CHOCTAWHATCHEE RIVER AT MOUTH

02366836 BLACK CREEK NR BRUCE

02366859 PATE BRANCH NR FREEPORT

PATE BRANCH AT MOUTH

02367365 TOMS CREEK $\begin{array}{lcccc}\text { SITE } & \text { AREA } & \text { AREA } & \text { LAT } & \text { LONG } \\ \text { TYPE } & \text { SQ.MI. } & \text { QUALIFIER DG/MI/SD DG/MI/SO }\end{array}$

QUALIFIER DG/MI/SD DG/MI/SD QUADRANGLE NAME

CNTY

CARYVILLE

059

176.00

30/46/32 85/49/40 CARYVILLE

059

SW $3,499.00$

51.80

30/45/49 86/01/22 GLENDALE

059

30/43/28 85/56/12 PONCE DE LEON

059

30/43/29 85/56/07 PONCE DE LEON

30/43/16 85/55/58 PONCE DE LEON

30/37/51 85/54/45 PONCE DE LEON

30/41/53 86/04/50 DEFUNIAK SPGS E 131

30/36/51 86/00/50 ROCK HILL

$30 / 36 / 48$ 85/54/50 REDBAY

30/32/18 85/55/14 REDBAY

30/32/06 85/52/49 REDBAY

30/37/35 85/42/45 POPLAR HEAD

30/35/08 85/48/05 MILLERS FERRY

$30 / 36 / 3185 / 48 / 21$ MILLERS FERRY

30/27/03 85/53/54 BRUCE

30/25/08 85/52/18 REDHEAD

005

30/24/12 86/07/26 BUNKER

30/28/26 85/59/20 BRUCE

$30 / 28 / 44$ 86/04/25 BUNKER

131

30/28/15 86/04/13 BUNKER

$30 / 30 / 2286 / 31 / 27$ VALPARAISO

091 
DRAINAGE AREAS FOR SELECTED STREAM SITES IN FLORIDA

CHOCTAWHATCHEE, YELLOW. AND ESCAMBIA RIVERS PEROIDO RIVER BASIN

SITE

NUMBER

NAME AND LOCATION

02376108 ELEVENMILE CREEK NR ENSLEY

02376300 BRUSHY CREEK NR WALNUT HILL

02376400 MCDAVID CREEK NR BARRINEAU PARK

02376500 PERDIDO RIVER AT BARRINEAU PARK

02376551 CHURCHHOUSE BRANCH NR BARRINEAU PARK

CHURCHHOUSE BRANCH AT MOUTH

02376700 JACKS BRANCH NR MUSCOGEE

PERDIDO RIVER AT MOUTH
NAME AND LOCATION

02364620 EIGHTMILE CREEK NR GASKIN

02364769 SPRING BRANCH NR GASKIN

02364781 LIMESTONE CREEK NR GASKIN

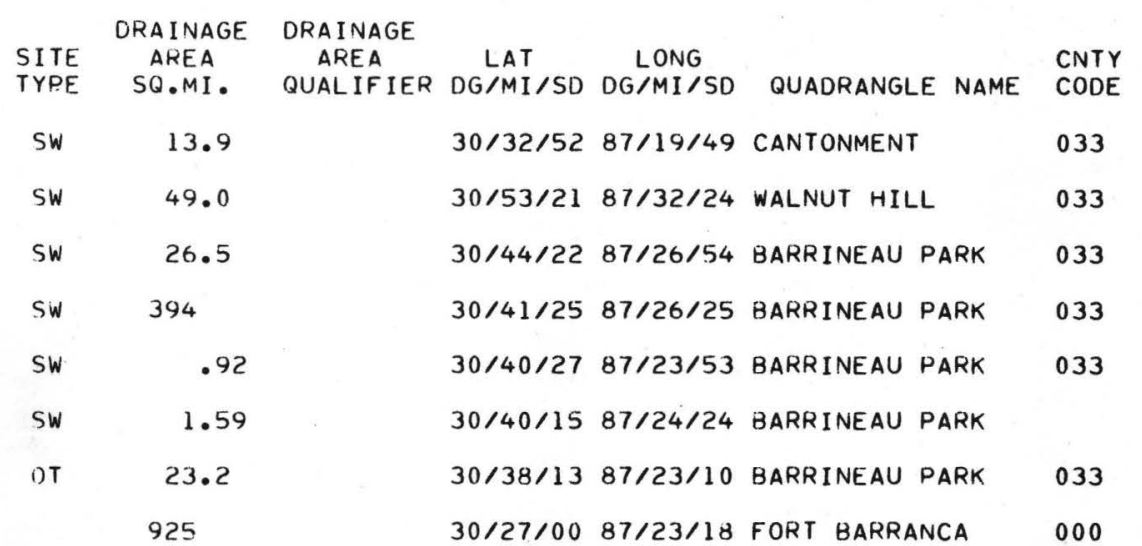

PEA RIVER

\begin{tabular}{|c|c|c|c|c|c|c|c|}
\hline $\begin{array}{l}\text { SITE } \\
\text { TYPE }\end{array}$ & $\begin{array}{l}\text { DRAINAGE } \\
\text { AREA } \\
\text { SQ.MI. }\end{array}$ & $\begin{array}{l}\text { DRA INAGE } \\
\text { AREA } \\
\text { QUALIF IER }\end{array}$ & $\begin{array}{l}\text { LAT } \\
D G / M I / S D\end{array}$ & $\begin{array}{c}\text { LONG } \\
\text { DG/MI/SD }\end{array}$ & QUADRANGLE & NAME & $\begin{array}{l}\text { CNTY } \\
\text { CODE }\end{array}$ \\
\hline SW & 24.9 & & $30 / 58 / 50$ & $86 / 10 / 45$ & GASKIN & & 131 \\
\hline SW & 4.13 & & $30 / 58 / 18$ & $86 / 05 / 53$ & & & 131 \\
\hline s & 30 & APPROX & $30 / 59 / 10$ & $86 / 02 / 41$ & DARL INGTON & & 131 \\
\hline
\end{tabular}

CHOCTAWHATCHEE RIVER BELOW PEA RIVER

\begin{tabular}{|c|c|c|c|c|c|c|c|}
\hline $\begin{array}{l}\text { SITE } \\
\text { TYPE }\end{array}$ & $\begin{array}{c}\text { DRAINAGE } \\
\text { AREA } \\
\text { SQ.MI. }\end{array}$ & $\begin{array}{l}\text { DRAINAGE } \\
\text { AREA } \\
\text { QUALIFIER }\end{array}$ & $\begin{array}{c}\text { LAT } \\
D G / M I / S O\end{array}$ & $\begin{array}{c}\text { LONG } \\
D G / M I / S D\end{array}$ & QUADRANGLE & NAME & $\begin{array}{l}\text { CNTY } \\
\text { CODE }\end{array}$ \\
\hline SW & 3,209 & & $30 / 56 / 59$ & $85 / 50 / 35$ & IZAGORA & & 059 \\
\hline SW & 5.09 & & $30 / 55 / 17$ & $85 / 56 / 01$ & HOBBS CROSS & ROADS & 059 \\
\hline SW & 5.36 & & $30 / 54 / 55$ & $85 / 55 / 38$ & HOBBS CROSS & ROADS & \\
\hline SW & 166 & & $30 / 49 / 37$ & $85 / 48 / 33$ & CARYVILLE & & 059 \\
\hline Sw & 6.88 & & $30 / 48 / 46$ & $85 / 48 / 06$ & CARYVILLE & & 059 \\
\hline SW & 7.12 & & $30 / 48 / 37$ & $85 / 49 / 02$ & & & 059 \\
\hline
\end{tabular}


DRAINAGE AREAS FOR SELECTED STREAM SITES IN FLORIDA

CHOCTAWHATCHEE, YELLOW, AND ESCAMBIA RIVERS

ESCAMBIA RIVER

SI TE
NUMBER

NAME ANO LOCATION

02375500 ESCAMBIA RIVER NR CENTURY

02375660 CANOE CREEK NR BLUFF SPRINGS

02375800 MOORE CREEK NR CHUMUCKLA

02376000 PINE BARREN CREEK NR BARTH

PINE BARREN CREEK AT MOUTH

02376033 ESCAMBIA RIVER NR MOLINO

02376039 ESCAMBIA RIVER NR COTTAGE HILL

02376047 ESCAMBIA RIVER NK GONZALES

02376050 ESCAMBIA RIVER NR PACE

ESCAMBIA RIVER AT MOUTH
02376077 CARPENTER CREEK NR PENSACOLA

\begin{tabular}{|c|c|c|c|c|c|c|}
\hline $\begin{array}{l}\text { SITE } \\
\text { TYPE }\end{array}$ & $\begin{array}{c}\text { DRAINAGE } \\
\text { AREA } \\
\text { SQ.MI. }\end{array}$ & $\begin{array}{l}\text { DRA INAGE } \\
\text { AREA } \\
\text { QUAL IF IER }\end{array}$ & $\begin{array}{l}\text { LAT } \\
D G / M I / S D\end{array}$ & $\begin{array}{c}\text { LONG } \\
\mathrm{DG} / \mathrm{MI} / \mathrm{SD}\end{array}$ & QUADRANGLE NAME & $\begin{array}{l}\text { CNTY } \\
\text { CODE }\end{array}$ \\
\hline SW & $3,817.00$ & & $30 / 57 / 25$ & $87 / 14 / 00$ & ALLENTON & 113 \\
\hline SW & 35.00 & & $30 / 58 / 01$ & $87 / 21 / 43$ & GASKIN & 033 \\
\hline SW & 22.00 & & $30 / 48 / 35$ & $87 / 15 / 14$ & MCDAVID & 113 \\
\hline SW & $75 \cdot 30$ & & $30 / 47 / 55$ & $87 / 22 / 05$ & PENSACOLA & 033 \\
\hline SW & 98.10 & & $30 / 44 / 03$ & $87 / 18 / 45$ & MCDAVID & \\
\hline SW & 4.147 .00 & & $30 / 40 / 05$ & $87 / 16 / 00$ & DOGWOOD CREEK & 033 \\
\hline SW & 4.172 .00 & & $30 / 35 / 03$ & $87 / 15 / 25$ & DOGWOOD CREEK & 033 \\
\hline SW & $4,183.00$ & & $30 / 36 / 42$ & $87 / 15 / 32$ & CANTONMENT & 033 \\
\hline SW & 4.183 .00 & & $30 / 35 / 58$ & $87 / 14 / 48$ & MILTON SOUTH & 113 \\
\hline SW & $4,233.00$ & & $30 / 32 / 32$ & $87 / 10 / 12$ & PACE & \\
\hline SW & 4.6 & & $30 / 29 / 02$ & $87 / 13 / 22$ & PENSACOLA & 033 \\
\hline
\end{tabular}



DRAINAGE AREAS FOR SELECTED LAKE SITES IN FLORIDA 
DRAINAGE AREAS FOR SELECTED LAKE SITES IN FLORIDA

ALTAMAHA/ST. MARYS RIVERS

ST. MARYS RIVER BASIN

SITE
NUMBER

NAME AND LOCATION

02228700 OCEAN POND AT OLUSTEE

SITE

NUMBER

NAME AND LOCATION

02231400 BLUE CYPRESS LAKE NR FELLSMERE.

02232100 LAKE WASHINGTON NR EAU GALLIE

02232300 LAKE POINSETT NR COCOA

02232420 CLEAR LAKF. NR COCOA

02233448 LAKE SPIER NR ORLANDO

02233450 LAKE CORRINE NR ORLANDO

02233755 LAKE CATHERINE NR CHULUOTA

02233900 LAKE GENEVA AT GENEVA

02234160 LAKE WINNEMISSETT NR DE LAND

02234200 SPRING LAKE. AT ORLANDO

02234205 LAKE ADAIR AT ORLANDO

02234210 PARK LAKE AT ORLANDO

02234215 LAKE CONCORD AT ORLANDO

02234220 LAKE HIGHLAND AT ORLANDO

02234225 LAKE IVANHOE AT ORLANDO

02234240 LAKE ROWENA AT ORLANDO

02234261 LAKE SUE AT ORLANDO

02234294 LAKE CHARITY AT MAITLAND

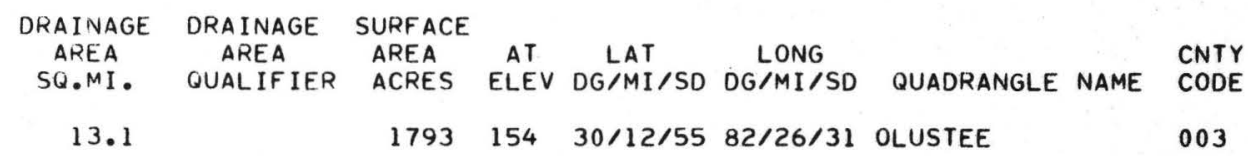

ST. JOHNS RIVER

ST. JOHNS RIVER BASIN ABOVE OKLAWAHA RIVER

\begin{tabular}{|c|c|c|c|c|c|c|c|}
\hline $\begin{array}{l}\text { DRAINAGE } \\
\text { AREA } \\
\text { SQ.MI. }\end{array}$ & $\begin{array}{l}\text { DRA INAGE } \\
\text { AREA } \\
\text { QUAL IF IER }\end{array}$ & $\begin{array}{l}\text { SURFACE } \\
\text { AREA } \\
\text { ACRES }\end{array}$ & $\begin{array}{c}\text { AT } \\
\text { ELEV }\end{array}$ & $\begin{array}{l}\text { LAT } \\
\mathrm{DG} / \mathrm{MI} / \mathrm{SD}\end{array}$ & $\begin{array}{c}\text { LONG } \\
\mathrm{DG} / \mathrm{MI} / \mathrm{SO}\end{array}$ & QUADRANGLE NAME & $\begin{array}{l}\text { CNTY } \\
\text { CODE }\end{array}$ \\
\hline 489 & & 6522 & 24 & $27 / 43 / 35$ & $80 / 46 / 30$ & FORT DRUM NE & 061 \\
\hline 1,025 & & 2665 & 17 & $28 / 09 / 09$ & $80 / 44 / 21$ & EAU GALLIE & 009 \\
\hline 1,272 & & 4293 & 12 & $28 / 21 / 01$ & $80 / 48 / 56$ & LAKE POINSETT & 009 \\
\hline .26 & & 14 & 23 & $28 / 23 / 04$ & $80 / 45 / 32$ & SHARPES & 009 \\
\hline .60 & & 77 & 96 & $28 / 33 / 50$ & $81 / 19 / 27$ & ORLANDO EAST & 095 \\
\hline .34 & & 24 & 90 & $28 / 34 / 43$ & $81 / 19 / 40$ & ORLANDO EAST & 095 \\
\hline 2.09 & & 193 & 91 & $28 / 34 / 28$ & $81 / 19 / 37$ & ORLANDO EAST & 095 \\
\hline .15 & & 14 & 53 & $28 / 38 / 33$ & $81 / 07 / 36$ & OVIEDO & 117 \\
\hline .46 & & 29 & 2 & $28 / 44 / 35$ & $81 / 06 / 25$ & GENEVA & 117 \\
\hline 1.10 & & 169 & 58 & $29 / 01 / 10$ & $81 / 15 / 06$ & DE LAND & 127 \\
\hline .52 & & 35 & 8 & $28 / 33 / 27$ & $81 / 23 / 48$ & ORLANDO WEST & 095 \\
\hline 1.27 & & 22 & 77 & $28 / 33 / 29$ & $81 / 23 / 20$ & ORLANDO WEST & 095 \\
\hline .12 & & 7 & 88 & $28 / 33 / 28$ & $81 / 22 / 21$ & ORLANDO EAST & 095 \\
\hline 2.10 & & 70 & 78 & $28 / 33 / 26$ & $81 / 23 / 17$ & ORLANDO WEST & 095 \\
\hline .22 & & 30 & 77 & $28 / 33 / 36$ & $81 / 22 / 24$ & ORLANDO EAST & 095 \\
\hline 3.27 & & 122 & 78 & $28 / 33 / 26$ & $81 / 22 / 34$ & ORLANDO WEST & 095 \\
\hline 5.13 & & 57 & 73 & $28 / 34 / 19$ & $81 / 21 / 48$ & ORLANDO EAST & 095 \\
\hline 6.34 & & 144 & 73 & $28 / 34 / 52$ & $81 / 21 / 24$ & ORLANDO EAST & 095 \\
\hline .66 & & 63 & 69 & $28 / 38 / 11$ & $81 / 22 / 46$ & FOREST CITY & 095 \\
\hline
\end{tabular}


DRAINAGE AREAS FOR SELECTED LAKE SITES IN FLORIDA

ST. JOHNS RIVER BASIN ABOVE OKLAWAHA RIVER

SI TE
NUMBER

NAME AND LOCATION

02234296 LAKE FAITH AT MAITLAND

02234297 LAKE HOPE AT MAITLAND

02234300 LAKE MAITLAND AT WINTER PARK

02234318 LAKE HOWELL NR CASSELBERRY

02234369 GRACE LAKE AT LONGWOOD

02234373 LAKE MYRTLE NR LONGWOOD

02234394 ISLAND LAKE AT LONGWOOD

02234398 LAKE KATHRYN AT CASSELBERRY

02234409 LAKE ADA AT SANFORD

02234414 LAKE MARY AT LAKE MARY

02234434 LAKE JESSUP NR SANFORD

02234450 LAKE DUPONT NR LAKE HELEN

02234499 LAKE MONROE NR SANFORD

02234638 LAKE BRANTLEY NR FOREST CITY

02234800 SILVER LAKE AT ORLANDO

02234810 LITTLE LAKE FAIRVIEW AT ORLANDO

02234812 LAKE FAIRVIEW AT ORLANDO

02234814 LAKE WEKIVA NR MAITLAND

02234900 HERRICK LAKE NR ORLANDO

02234930 LAKE SHERWOOD NR ORLANDO

02234935 LAKE JOHIO NR OCOEE

02234942 BEAR LAKE NR FOREST CITY

02234943 LAKE ORIENTA AT ALTAMONTE SPRINGS

\begin{tabular}{|c|c|c|c|c|c|c|c|}
\hline $\begin{array}{l}\text { DRAINAGE } \\
\text { AREA } \\
\text { SQ.MI. }\end{array}$ & $\begin{array}{l}\text { DRA INAGE } \\
\text { AREA } \\
\text { QUAL IF IER }\end{array}$ & $\begin{array}{l}\text { SURFACE } \\
\text { AREA } \\
\text { ACRES }\end{array}$ & $\begin{array}{l}\text { AT } \\
\text { ELEV }\end{array}$ & $\begin{array}{c}\text { LAT } \\
D G / M I / S D\end{array}$ & $\begin{array}{c}\text { LONG } \\
\mathrm{DG} / \mathrm{MI} / \mathrm{SO}\end{array}$ & QUADRANGLE NAME & $\begin{array}{l}\text { CNTY } \\
\text { CODE }\end{array}$ \\
\hline .71 & & 33 & 68 & $28 / 38 / 22$ & $81 / 22 / 08$ & CASSELBERRY & 095 \\
\hline 1.67 & & 36 & 67 & $28 / 38 / 24$ & $81 / 22 / 15$ & CASSELBERRY & 095 \\
\hline 20.6 & & 452 & 66 & $28 / 36 / 49$ & $81 / 20 / 35$ & ORLANDO EAST & 095 \\
\hline 27.8 & & 406 & 53 & $28 / 38 / 33$ & $81 / 18 / 46$ & CASSELBERRY & 117 \\
\hline .67 & & 31 & 61 & $28 / 43 / 00$ & $81 / 22 / 36$ & FOREST CITY & 117 \\
\hline 1.76 & & 55 & 4 & $28 / 43 / 22$ & $81 / 21 / 40$ & CASSELBERRY & 117 \\
\hline 1.29 & & 204 & 83 & $28 / 41 / 17$ & $81 / 21 / 42$ & CASSELBERRY & 117 \\
\hline 8.10 & & 80 & 52 & $28 / 40 / 47$ & $81 / 19 / 51$ & CASSELBERRY & 117 \\
\hline .32 & & 49 & 42 & $28 / 46 / 05$ & $81 / 17 / 00$ & SANF ORD & 117 \\
\hline$\bullet 88$ & & 154 & 40 & $28 / 45 / 09$ & $81 / 19 / 13$ & SANFORD & 117 \\
\hline .11 & & 20 & 45 & $28 / 40 / 42$ & $81 / 11 / 55$ & OVIEDO & 117 \\
\hline 156 & & 7792 & 5 & $28 / 47 / 09$ & $81 / 10 / 50$ & OSTEEN & 117 \\
\hline 23.4 & & 220 & 17 & $28 / 55 / 12$ & $81 / 12 / 32$ & LAKE HELEN & 127 \\
\hline 2,582 & & 8840 & 5 & $28 / 50 / 13$ & $81 / 19 / 28$ & SANFORD & 117 \\
\hline 1.56 & & 282 & 48 & $28 / 41 / 28$ & $81 / 25 / 23$ & FOREST CITY & 117 \\
\hline .51 & & 69 & 92 & $28 / 34 / 31$ & $81 / 23 / 53$ & ORLANDO WEST & 095 \\
\hline 1.27 & & 86 & 89 & $28 / 35 / 29$ & $81 / 23 / 38$ & ORLANDO WEST & 095 \\
\hline 3.73 & & 403 & 88 & $28 / 35 / 22$ & $81 / 24 / 20$ & ORLANDO WEST & 095 \\
\hline 13.4 & & 190 & 86 & $28 / 36 / 10$ & $81 / 25 / 38$ & ORLANDO WEST & 095 \\
\hline 1.94 & & 39 & 70 & $28 / 32 / 49$ & $81 / 29 / 01$ & ORLANDO WEST & 095 \\
\hline 17.1 & & 120 & 68 & $28 / 33 / 11$ & $81 / 29 / 37$ & ORLANDO WEST & 095 \\
\hline .32 & & 27 & 111 & $28 / 34 / 41$ & $81 / 30 / 37$ & WINTER GARDEN & 095 \\
\hline 1.59 & & 305 & 104 & $28 / 39 / 20$ & $81 / 26 / 43$ & FOREST CITY & 117 \\
\hline 1.61 & & 130 & 61 & $28 / 39 / 17$ & $81 / 22 / 46$ & FOREST CITY & 117 \\
\hline
\end{tabular}


DRAINAGE AREAS FOR SELECTEO LAKE SITES IN FLORIDA

ST. JOHNS RIVER BASIN ABOVE OKLAWAHA RIVER

SITE

NAME ANO LOCATION

02234995 ELEVENTH HOLE POND AT ALTAMONTE SPRINGS

02234999 LINDEN LAKE NR LAKE MARY

LAKE MARKHAM NR PAOLA

02235010 SYLVAN LAKE NR PAOLA

02235150 LAKE DORR NR ALTOONA

02235900 PINE LAKE NR CASSIA

02236190 LAKE DELANCY NR EUREKA

02236200 LAKE KERR NR EUREKA

02236210 LAKE GEORGE NR SALT SPRINGS

$\stackrel{\infty}{\infty}$

\begin{tabular}{|c|c|c|c|c|c|c|c|}
\hline $\begin{array}{l}\text { DRAINAGE } \\
\text { AREA } \\
\text { SQ.MI. }\end{array}$ & $\begin{array}{l}\text { DRA INAGE } \\
\text { AREA } \\
\text { QUALIF IER }\end{array}$ & $\begin{array}{l}\text { SURFACE } \\
\text { AREA } \\
\text { ACRES }\end{array}$ & $\begin{array}{l}\text { AT } \\
\text { ELEV }\end{array}$ & $\begin{array}{c}\text { LAT } \\
\mathrm{DG} / \mathrm{MI} / \mathrm{SD}\end{array}$ & $\begin{array}{c}\text { LONG } \\
\mathrm{DG} / \mathrm{MI} / \mathrm{SD}\end{array}$ & QUADRANGLE NAME & $\begin{array}{l}\text { CNTY } \\
\text { CODE }\end{array}$ \\
\hline 1.25 & & 1 & 38 & $28 / 41 / 05$ & $81 / 22 / 48$ & FOREST CITY & 117 \\
\hline .62 & & 14 & 42 & $28 / 45 / 20$ & $81 / 22 / 41$ & SANFORD SW & 117 \\
\hline .98 & & 83 & 44 & $28 / 47 / 56$ & $81 / 23 / 37$ & SANFORD SW & 117 \\
\hline 3.43 & & 190 & 40 & $28 / 48 / 26$ & $81 / 23 / 09$ & SANFORD SW & 117 \\
\hline 26.5 & & 1712 & 34 & $29 / 00 / 10$ & $81 / 38 / 05$ & FARLES LAKE & 069 \\
\hline 1.79 & & 27 & 37 & $28 / 56 / 10$ & $81 / 26 / 01$ & PINE LAKES & 069 \\
\hline 30.0 & & 781 & 22 & $29 / 25 / 30$ & $81 / 45 / 40$ & LAKE DELANCY & 083 \\
\hline 102 & & 4484 & 25 & $29 / 20 / 10$ & $81 / 46 / 00$ & LAKE KERR & 083 \\
\hline 3,721 & & 46780 & 4 & $29 / 17 / 44$ & $81 / 39 / 06$ & SALT SPRINGS & 083 \\
\hline
\end{tabular}

OKLAWAHA RIVER BASIN

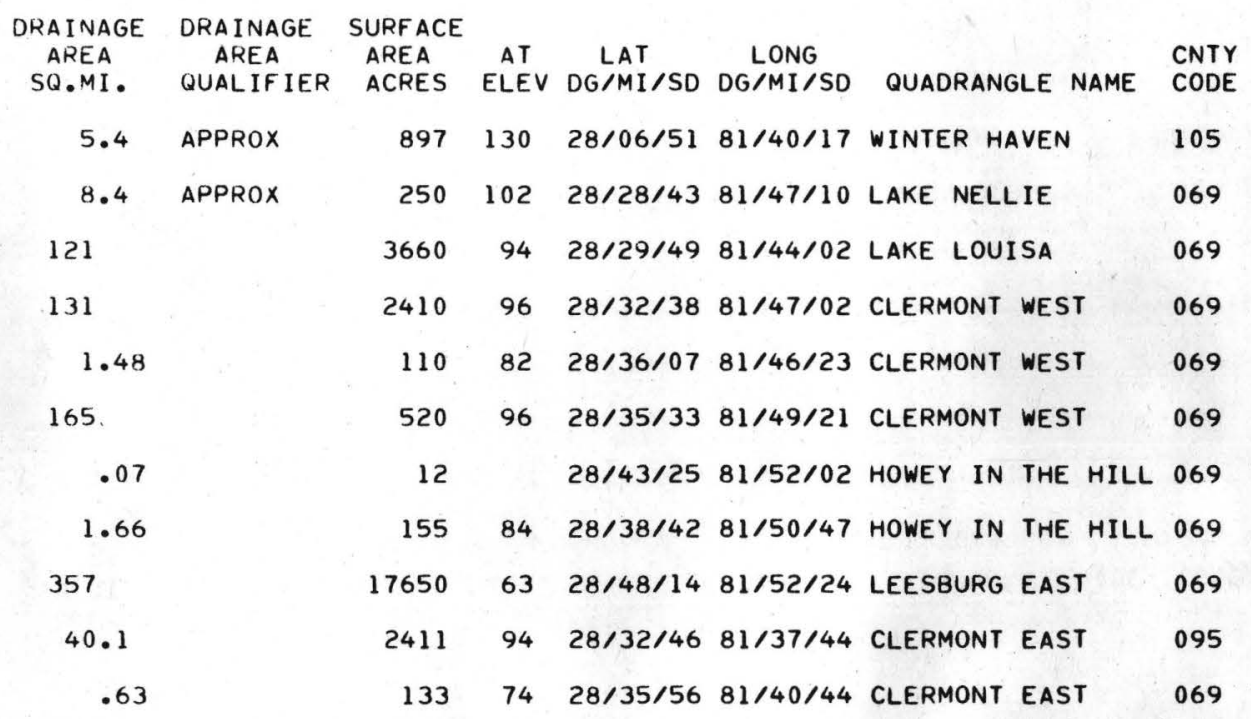


SITE
NIJMBER

NAME AND LOCATION

$02 ? 37600$ LAKE APOPKA AT WINTER GARDEN

02237660 LAKE FRANCIS NR PLYMOUTH

02.237752 LAKE DICIE AT EUSTIS

02237753 WEST CROOKED LAKE NR EUSTIS

02237800 LAKE DORA AT MOUNT DORA

02237865 LAKE UMATILLA AT UMATILLA

02237900 LAKE EUSTIS AT EUSTIS

02238170 NICOTOON LAKE NR ALTOONA

02238180 HOLLY LAKE NR UMATILLA

02238200 LAKE YALE AT GRAND ISLAND

\begin{tabular}{|c|c|c|c|c|c|c|c|}
\hline $\begin{array}{l}\text { DRAINAGE } \\
\text { AREA } \\
\text { SQ.MI. }\end{array}$ & $\begin{array}{c}\text { DRA INAGE } \\
\text { AREA } \\
\text { QUAL IF IER }\end{array}$ & $\begin{array}{l}\text { SURFACE } \\
\text { AREA } \\
\text { ACRES }\end{array}$ & $\begin{array}{l}\text { AT } \\
\text { ELEV }\end{array}$ & $\begin{array}{c}\text { LAT } \\
\mathrm{DG} / \mathrm{MI} / \mathrm{SO}\end{array}$ & $\begin{array}{c}\text { LONG } \\
\mathrm{DG} / \mathrm{MI} / \mathrm{SD}\end{array}$ & QUADRANGLE NAME & $\begin{array}{l}\text { CNTY } \\
\text { CODE }\end{array}$ \\
\hline 128 & & 30630 & 66 & $28 / 34 / 36$ & $81 / 35 / 13$ & WINTER GARDEN & 095 \\
\hline .67 & & 33 & 59 & $28 / 42 / 20$ & $81 / 32 / 23$ & APOPKA & 095 \\
\hline .11 & & 9 & & $28 / 49 / 55$ & $81 / 40 / 53$ & EUSTIS & 069 \\
\hline .67 & & 107 & 65 & $28 / 49 / 49$ & $81 / 40 / 20$ & EUSTIS & 069 \\
\hline 236 & & 4437 & 63 & $28 / 47 / 46$ & $81 / 38 / 39$ & EUSTIS & 069 \\
\hline 2.46 & & 165 & 69 & $28 / 55 / 09$ & $81 / 39 / 57$ & UMATILLA & 069 \\
\hline 646 & & 7806 & 63 & $28 / 51 / 08$ & $81 / 41 / 23$ & EUSTIS & 069 \\
\hline 19.20 & & 328 & 55 & $28 / 59 / 22$ & $81 / 43 / 25$ & UMATILLA & 083 \\
\hline .78 & & 96 & 59 & $28 / 56 / 11$ & $81 / 43 / 04$ & UMATILLA & 069 \\
\hline 67.6 & & 4030 & 59 & $28 / 52 / 52$ & $81 / 42 / 21$ & UMATILLA & 069 \\
\hline 775 & & 10660 & 59 & $28 / 50 / 29$ & $81 / 52 / 12$ & LEESBURG EAST & 069 \\
\hline 53.8 & & 5760 & 57 & $29 / 02 / 23$ & $81 / 55 / 44$ & LAKE WEIR & 083 \\
\hline 5.01 & & 482 & 55 & $29 / 04 / 12$ & $81 / 59 / 25$ & LAKE WEIR & 083 \\
\hline 8.48 & & 633 & 57 & $29 / 02 / 23$ & $81 / 57 / 44$ & LAKE WEIR & 083 \\
\hline 9.86 & & 1260 & 54 & $29 / 08 / 21$ & $81 / 51 / 03$ & HALFMOON LAKE & 083 \\
\hline 39.4 & & 346 & & $29 / 11 / 10$ & $81 / 50 / 10$ & HALFMOON LAKE & 083 \\
\hline 114 & & 7350 & 68 & $29 / 39 / 04$ & $82 / 14 / 22$ & ORANGE HEIGHTS & 001 \\
\hline 1.96 & & 73 & 68 & $29 / 38 / 20$ & $82 / 21 / 15$ & GAINESVILLE EAST & 001 \\
\hline 8.65 & & 204 & 57 & $29 / 36 / 44$ & $82 / 24 / 14$ & ARREDONDO & 001 \\
\hline 3.00 & & 177 & 63 & $29 / 37 / 16$ & $82 / 20 / 32$ & MICANOPY & 001 \\
\hline 88.0 & & 8800 & 58 & $29 / 30 / 19$ & $82 / 06 / 08$ & HAWTHORNE & 001 \\
\hline 1,012 & & 13160 & 58 & $29 / 25 / 37$ & $82 / 12 / 26$ & MC INTOSH & 001 \\
\hline
\end{tabular}

02238800 LAKE WEIR AT OKLAWAHA

02238820 SMITH LAKE NR CANOLER

02238830 BOWERS LAKE NR OKLAWAHA

02240200 LAKE RRYANT NR SILVER SPRINGS

02240210 MILL DAM LAKE NR LYNNE.

02240900 NEWNANS LAKE NR GAINESVILLE

02240952 LAKE ALICE AT GAINESVILLE

02240958 LAKE KANAPAHA AT ARREDONDO

02240980 BIVANS ARM NR GAINESVILLE

02242400 LOCHLOOSA LAKE. NR LOCHLOOSA

02242450 ORANGE LAKE AT ORANGE LAKE 
DRAINAGE AREAS FOR SELECTED LAKE SITES IN FLORIDA

ST. JOHNS RIVER
ST. JOHNS RIVER BASIN BELOW OKLAWAHA RIVER

SITE

NUMBER

NAME AND LOCATION

02244350 LAKE WINONA NR DE. LAND

02244370 LAKE HIRES NR DE LAND

CRESCENT LAKE

02244550 BLUE POND NR KEYSTONE HEIGHTS

02244600 SAND HILL LAKE NR KEYSTONE HEIGHTS

02244650 MAGNOLIA LAKE NR KEYSTONE HEIGHTS

02244700 LOCH LOMMOND NR KEYSTONE HEIGHTS

02244750 BROOKLYN LAKE AT KEYSTONE HEIGHTS

02244800 LAKE GENEVA AT KEYSTONE HEIGHTS

02244850 PEBBLE LAKE NR KEYSTONE HEIGHTS

02244905 LAKE JOHNSON NR KEYSTONE HEIGHTS (BIG LAKE)

02244950 LAKE GRANDIN NR INTERLACHEN

02245700 KINGSLEY LAKE AT CAMP BLANDING

SITE

NUMBER

NAME AND LOCATION

02260800 ALLIGATOR LAKE NR ASHTON

02261900 LAKE MARY JANE. NR NARCOOSSEE

02262200 LAKE HART NR NARCOOSSEE

02262550 LAKE UNDERHILL AT ORLANDO

02262700 BASS LAKE NR ORLANDO

02262800 LAKE CONWAY AT PINE CASTLE

02263400 EAST LAKE TOHOPEKALIGA AT ST CLOUD

02263750 BIG SANO LAKE AT DOCTOR PHILLIPS

\begin{tabular}{|c|c|c|c|c|c|c|c|}
\hline $\begin{array}{l}\text { DRAINAGE } \\
\text { AREA } \\
\text { SQ.MI. }\end{array}$ & $\begin{array}{l}\text { DRAINAGE } \\
\text { AREA } \\
\text { QUALIFIER }\end{array}$ & $\begin{array}{l}\text { SURFACE } \\
\text { AREA } \\
\text { ACRES }\end{array}$ & $\begin{array}{l}\text { AT } \\
\text { ELEV }\end{array}$ & $\begin{array}{c}\text { LAT } \\
D G / M I / S D\end{array}$ & $\begin{array}{c}\text { LONG } \\
\mathrm{DG} / \mathrm{MI} / \mathrm{SD}\end{array}$ & QUADRANGLE NAME & $\begin{array}{l}\text { CNTY } \\
\text { CODE }\end{array}$ \\
\hline 1.35 & & 156 & 36 & $29 / 10 / 50$ & $81 / 20 / 06$ & LAKE DIAS & 127 \\
\hline 2.32 & & 73 & 42 & $29 / 07 / 32$ & $81 / 18 / 02$ & LAKE DIAS & 127 \\
\hline 456 & & 17140 & 1 & & & & \\
\hline 2.32 & & 201 & 176 & $29 / 52 / 40$ & $82 / 01 / 50$ & STARKE & 019 \\
\hline 11.0 & & 1250 & 128 & $29 / 51 / 01$ & $82 / 01 / 10$ & KEYSTONE HEIGHTS & 019 \\
\hline 14.4 & & 201 & 118 & $29 / 49 / 12$ & $82 / 00 / 55$ & KEYSTONE HEIGHTS & 019 \\
\hline .38 & & 17 & 102 & $29 / 48 / 15$ & $82 / 00 / 50$ & KEYSTONE HEIGHTS & 019 \\
\hline 17.4 & & 635 & 112 & $29 / 47 / 46$ & $82 / 01 / 21$ & KEYSTONE HEIGHTS & 019 \\
\hline 35.5 & & 1746 & 108 & $29 / 46 / 26$ & $82 / 01 / 59$ & KEYSTONE HEIGHTS & 019 \\
\hline .19 & & 10 & & $29 / 49 / 31$ & $81 / 57 / 10$ & GOLD HEAD BRANCH & 019 \\
\hline 6.37 & & & & $29 / 49 / 26$ & $81 / 56 / 42$ & GOLD HEAD BRANCH & 019 \\
\hline 3.71 & & 354 & & $29 / 40 / 16$ & $81 / 52 / 55$ & PUTNAM HALL & 107 \\
\hline 6.84 & & 1627 & 176 & $29 / 58 / 28$ & $81 / 59 / 22$ & KINGSLEY & 019 \\
\hline
\end{tabular}

KISSIMMEE RIVER BASIN

\begin{tabular}{|c|c|c|c|c|c|c|c|}
\hline $\begin{array}{l}\text { DRAINAGE } \\
\text { AREA } \\
\text { SQ.MI. }\end{array}$ & $\begin{array}{l}\text { DRA INAGE } \\
\text { AREA } \\
\text { QUALIF IER }\end{array}$ & $\begin{array}{l}\text { SURFACE } \\
\text { AREA } \\
\text { ACRES }\end{array}$ & $\begin{array}{c}\text { AT } \\
\text { ELEV }\end{array}$ & $\begin{array}{c}\text { LAT } \\
D G / M I / S D\end{array}$ & $\begin{array}{c}\text { LONG } \\
\mathrm{DG} / \mathrm{MI} / \mathrm{SD}\end{array}$ & QUADRANGLE NAME & $\begin{array}{l}\text { CNTY } \\
\text { CODE }\end{array}$ \\
\hline 26.6 & & 3401 & 64 & $28 / 13 / 55$ & $81 / 11 / 17$ & ASHTON & 097 \\
\hline 124 & & 1161 & 60 & $28 / 22 / 46$ & $81 / 11 / 15$ & NARCOOSEE NW & 095 \\
\hline 166 & & 1841 & 58 & $28 / 22 / 46$ & $81 / 13 / 27$ & NARCOOSSEE & 095 \\
\hline 1.49 & & & & $28 / 32 / 26$ & $81 / 19 / 59$ & ORLANDO EAST & 095 \\
\hline .83 & & 22 & 90 & $28 / 30 / 36$ & $81 / 20 / 35$ & ORLANDO EAST & 095 \\
\hline 12.7 & & 1079 & 86 & $28 / 27 / 55$ & $81 / 21 / 23$ & PINE CASTLE & 095 \\
\hline 308 & & 11950 & 57 & $28 / 15 / 30$ & $81 / 16 / 57$ & ST CLOUD NORTH & 097 \\
\hline 8.99 & & 1106 & 94 & $28 / 26 / 20$ & $81 / 29 / 50$ & LAKE JESSAMINE & 095 \\
\hline
\end{tabular}


DRAINAGE AREAS FOR SELECTED LAKE SITES IN FLORIDA

SOUTHERN FLORIDA

KISSIMMEE RIVER BASIN

SI TE
NUMBER

NAME AND LOCATION

02263776 LAKE BRYAN NR VINELAND

02263850 BAY LAKE NR VINELAND

02263864 LAKE MABEL NR DOCTOR PHILLIPS

02263890 LAKE BESSIE AT WINDERMERE

02263900 LAKE BUTLER AT WINDERMERE

02264900 LAKE TOHOPEKALIGA AT KISSIMMEE

02265400 LAKE GENTRY NR ST CLOUD

02266239 TROUT LAKE NR CLERMONT

02266600 CYPRESS LAKE NR ST CLOUD

02266650 LAKE MARION NR HAINES CITY

\begin{tabular}{|c|c|c|c|c|c|c|c|}
\hline $\begin{array}{l}\text { DRAINAGE } \\
\text { AREA } \\
\text { SQ.MI. }\end{array}$ & $\begin{array}{l}\text { DRA INAGE } \\
\text { AREA } \\
\text { QUALIFIEK }\end{array}$ & $\begin{array}{l}\text { SURFACE } \\
\text { AREA } \\
\text { ACRES }\end{array}$ & $\begin{array}{l}\text { AT } \\
\text { ELEV }\end{array}$ & $\begin{array}{l}\text { LAT } \\
D G / M I / S D\end{array}$ & $\begin{array}{l}\text { LONG } \\
\mathrm{DG} / \mathrm{MI} / \mathrm{SD}\end{array}$ & QUADRANGLE NAME & $\begin{array}{l}\text { CNTY } \\
\text { CODE }\end{array}$ \\
\hline 2.70 & & 210 & 98 & $28 / 21 / 46$ & $81 / 29 / 57$ & KISSIMMEE & 095 \\
\hline 14.8 & & 436 & 94 & $28 / 24 / 08$ & $81 / 33 / 28$ & WI NOERMERE & 095 \\
\hline 9.54 & & 397 & & $28 / 25 / 11$ & $81 / 32 / 58$ & WINDERMERE & 095 \\
\hline .64 & & 160 & 96 & $28 / 29 / 20$ & $81 / 31 / 50$ & WINOERMERE & 095 \\
\hline 14.5 & & 1665 & 98 & $28 / 29 / 26$ & $81 / 32 / 04$ & WINDERMERE & 095 \\
\hline 620 & APPROX & 18790 & 53 & $28 / 17 / 20$ & $81 / 24 / 20$ & KISSIMMEE & 097 \\
\hline 44.6 & & 1797 & 62 & $28 / 07 / 34$ & $81 / 15 / 31$ & ST CLOUD SOUTH & 097 \\
\hline 1.31 & & 163 & & $28 / 27 / 04$ & $81 / 43 / 00$ & LAKE LOUISA & 069 \\
\hline 1,162 & & 4085 & 52 & $28 / 03 / 40$ & $81 / 19 / 58$ & CYPRESS LAKE & 097 \\
\hline 35.7 & & 2968 & 67 & $28 / 05 / 56$ & $81 / 31 / 51$ & DUNDEE & 105 \\
\hline 58.9 & & 3736 & 76 & $27 / 58 / 37$ & $81 / 32 / 33$ & LAKE WALES & 105 \\
\hline 1,162 & & 6636 & 52 & $28 / 00 / 00$ & $81 / 22 / 50$ & LAKE HATCHINEHA & 097 \\
\hline 2.42 & & 330 & 112 & $27 / 54 / 13$ & $81 / 34 / 44$ & LAKE WALES & 105 \\
\hline 93.5 & & 7555 & 62 & $27 / 48 / 50$ & $81 / 23 / 16$ & LAKE WEOHYAKAPKA & 105 \\
\hline 133 & & 4592 & 53 & $27 / 56 / 35$ & $81 / 25 / 28$ & HESPERIDES & 105 \\
\hline 49.6 & & 5727 & 59 & $27 / 52 / 09$ & $81 / 02 / 36$ & LAKE MARIAN SE & 097 \\
\hline 1,507 & & 34760 & 49 & $27 / 48 / 09$ & $81 / 11 / 50$ & LK WEOHYAKAPKA NE & 097 \\
\hline 28.9 & & 1192 & 87 & $27 / 40 / 45$ & $81 / 32 / 22$ & FROSTPROOF & 105 \\
\hline 31.3 & & 5533 & 118 & $27 / 49 / 39$ & $81 / 33 / 12$ & BABSON PARK & 105 \\
\hline $42.0^{6}$ & & 1194 & 103 & $27 / 45 / 15$ & $81 / 32 / 25$ & BABSON PARK & 105 \\
\hline $60 \cdot 9$ & & 3454 & 78 & $27 / 43 / 39$ & $81 / 28 / 21$ & LAKE ARBUCKLE & 105 \\
\hline 1.70 & & 3787 & 53 & $27 / 39 / 50$ & $81 / 22 / 41$ & LAKE ARBUCKLE & 105 \\
\hline 12.2 & & 795 & 106 & $27 / 35 / 05$ & $81 / 28 / 55$ & LK ARBUCKLE SW & 055 \\
\hline 15.6 & & 471 & 99 & $27 / 33 / 35$ & $81 / 27 / 15$ & LK ARBUCKLE SW & 055 \\
\hline 6.06 & & 465 & 107 & $27 / 31 / 09$ & $81 / 28 / 59$ & LK ARBUCKLE SW & 055 \\
\hline
\end{tabular}


DRAINAGE AREAS FOR SELECTED LAKE SITES IN FLORIDA

KISSIMMEE RIVER BASIN

SITE
NUMBER

NAME AND LOCATION

02270550 LAKE JACKSON AT SEBRING

02270650 LAKE JOSEPHINE NR DE SOTO CITY 02270700 LAKE ANNIE NR LAKE PLACID

02270750 LAKE PLACID NR LK PLACID

02270850 LAKE PEARL AT LAKE PLACID

02270900 LAKE SIRENA AT LAKE PLACIO

02270950 LAKE JUNE-IN-WINTER NR LAKE PLACID

02271200 LAKE FRANCIS NR LAKE PLACID

02271540 GRASSY LAKE NR LAKE PLACID

02271557 MIRROR LAKE NR LAKE PLACID 02271580 LAKE HUNTLEY NR LAKE PLACID 02271600 CLAY LAKE NR LAKE PLACID 02271620 LAKE APTHORPE NR LAKE PLACIO 02271700 LAKE ISTOKPOGA NR DE SOTO CITY

\section{SITE
NUMBER} NAME AND LOCATION

02276400 LAKE OKEECHOBEE

NAME AND LOCATION

DRAINAGE
AREA
SQ.MI.
14.0
46.3
5.72
20.2
.23
1.37
44.0
49.1
5.39
.62
.30
9.54
11.7
15.3
607

LAKE OKEECHOBEE

SURFACE

AREA AT LAT LONG $\begin{array}{cc}\text { AREA } & \text { AREA } \\ \text { QUALIFIER } & \text { ACRES }\end{array}$

CNTY

$124071 \quad 27 / 24 / 00 \quad 81 / 25 / 10$ SEBRING

$85 \quad 111 \quad 27 / 12 / 3581 / 20 / 57$ CHILDS 055

$33819227 / 15 / 4481 / 22 / 08$ LAKE PLACID 055

$6786 \quad 27 / 17 / 1081 / 21 / 40$ LAKE PLACID 055

$1498627 / 17 / 1081 / 22 / 00$ LAKE PLACIO 055

$36627327 / 19 / 1981 / 25 / 08$ LK JUNE IN WINTER 055

$53070 \quad 27 / 20 / 00 \quad 81 / 24 / 10$ LK JUNE IN WINTER 055

$51090 \quad 27 / 15 / 0081 / 20 / 17$ LAKE PLACID 055

9692 27/16/33 81/21/41 LAKE PLACID 055

5486 27/17/05 81/21/25 LAKE PLACID 055

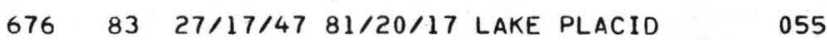

$3617827 / 18 / 3281 / 20 / 17$ LAKE PLACID 055

$2156927 / 20 / 3081 / 22 / 25$ LAKE PLACID 055

$27500 \quad 38 \quad 27 / 26 / 0981 / 17 / 05$ LORIDA

\begin{tabular}{|c|c|c|c|c|c|c|c|c|}
\hline $\begin{array}{l}\text { DRAINAGE } \\
\text { AREA } \\
\text { SQ.MI. }\end{array}$ & $\begin{array}{l}\text { DRA INAGE } \\
\text { AREA } \\
\text { QUAL IF IER }\end{array}$ & $\begin{array}{l}\text { SURFACE } \\
\text { AREA } \\
\text { ACRES }\end{array}$ & $\begin{array}{l}\text { AT } \\
\text { ELEV }\end{array}$ & $\begin{array}{c}\text { LAT } \\
D G / M I / S D\end{array}$ & $\begin{array}{c}\text { LONG } \\
D G / M I / S D\end{array}$ & QUADRANGLE & NAME & $\begin{array}{l}\text { CNTY } \\
\text { CODE }\end{array}$ \\
\hline 650 & & 436000 & 14 & $26 / 57 / 00$ & $80 / 50 / 00$ & OKEECHOBEE & & 093 \\
\hline
\end{tabular}

FLORIDA BAY AND THE FLORIDA KEYS

$\begin{array}{clcccc}\begin{array}{c}\text { DRAINAGE } \\ \text { AREA }\end{array} & \begin{array}{c}\text { DRAINAGE } \\ \text { AREA }\end{array} & \begin{array}{c}\text { SURFACÉ } \\ \text { AREA }\end{array} & \text { AT LAT LAT LONG } & \text { LONG } & \text { CNTY } \\ \text { SQ.MI. } & \text { QUALIFIER } & \text { ACRES } & \text { ELEV DG/MI/SD DG/MI/SO QUADRANGLE NAME } & \text { CODE }\end{array}$


SITE
NIJMBER

NAME AND LOCATION

02293461 LAKE ALFRED AT LAKE ALFRED)

02293478 LAKE ROCHELLE NR LAKE ALFRED

02293491 LAKE CONINE AT FLORENCE VILLA

02293496 LAKE SMART NR FLORENCE VILLA

D2293518 LAKE FANNIE NR FLORENCE VILLA

02293649 LAKE HENRY NR HAINES CITY

02293660 LAKE HAMILTON NR LAKE HAMILTON

02293670 LAKE OTIS AT WINTER HAVEN

02293728 LAKE ANNIE AT WAVERLY

02293774 MOUNTAIN LAKE NR LAKE WALES

PEACE RIVER BASIN

02293785 LAKE EFFIE AT LAKE. WALES

02293808 RUBY LAKE NR WAVERLY

02293823 LAKE MYRTLE NR LAKE WALES

02293999 LAKE MARIANA NR AUBURNDALE

02294008 LAKE JESSIE NR AUBURNDALE

02294010 LAKE HARTRIDGE AT WINTER HAVEN

02294028 DEER LAKE NR WINTER HAVEN

02294031 LAKE CANNON AT WINTER HAVEN

02294036 LAKE HOWARD AT WINTER HAVEN

02294041 LAKE SHIPP AT WINTER HAVEN

02294050 LAKE WINTERSET NR ELOISE

02294055 LAKE ELOISE NR ELOISE

02294065 LAKE LULU NR ELOISE

02294071 LAKE MCLEOD AT EAGLE LAKE

02294112 LAKE GARFIELD NR ALTURAS

\begin{tabular}{|c|c|c|c|c|c|c|c|}
\hline $\begin{array}{l}\text { DRAINAGE } \\
\text { AREA } \\
\text { SQ.MI. }\end{array}$ & $\begin{array}{l}\text { DRAINAGE } \\
\text { AREA } \\
\text { QUALIF IER }\end{array}$ & $\begin{array}{l}\text { SURFACE } \\
\text { AREA } \\
\text { ACRES }\end{array}$ & $\begin{array}{c}\text { AT } \\
\text { ELEV }\end{array}$ & $\begin{array}{c}\text { LAT } \\
D G / M I / S D\end{array}$ & $\begin{array}{l}\text { LONG } \\
\mathrm{DG} / \mathrm{MI} / \mathrm{SO}\end{array}$ & QUADRANGLE NAME & $\begin{array}{l}\text { CNTY } \\
\text { COOE }\end{array}$ \\
\hline 2.93 & & 736 & 129 & $28 / 05 / 48$ & $81 / 44 / 00$ & WINTER HAVEN & 105 \\
\hline 15.6 & & 581 & 128 & $28 / 04 / 10$ & $81 / 43 / 40$ & WINTER HAVEN & 105 \\
\hline 17.2 & & 237 & 128 & $28 / 03 / 08$ & $81 / 42 / 38$ & WINTER HAVEN & 105 \\
\hline 17.9 & & 279 & 128 & $28 / 03 / 08$ & $81 / 42 / 38$ & WINTER HAVEN & 105 \\
\hline 24.7 & & 833 & 125 & $28 / 03 / 08$ & $81 / 41 / 03$ & WINTER HAVEN & 105 \\
\hline 4.12 & & 861 & 126 & $28 / 04 / 50$ & $81 / 39 / 45$ & WINTER HAVEN & 105 \\
\hline 20.5 & & 2170 & 120 & $28 / 01 / 54$ & $81 / 38 / 42$ & WINTER HAVEN & 105 \\
\hline 1.00 & & 144 & 12 & $28 / 01 / 10$ & $81 / 42 / 35$ & WINTER HAVEN & 105 \\
\hline 4.06 & & 534 & 119 & $27 / 59 / 05$ & $81 / 36 / 40$ & LAKE WALES & 105 \\
\hline 2.30 & & 158 & 112 & $27 / 56 / 01$ & $81 / 34 / 59$ & LAKE WALES & 105 \\
\hline 2.51 & & 102 & 117 & $27 / 54 / 31$ & $81 / 36 / 11$ & LAKE WALES & 105 \\
\hline .93 & & 253 & 125 & $27 / 58 / 27$ & $81 / 39 / 30$ & ELOISE & 105 \\
\hline 4.98 & & 416 & 118 & $27 / 57 / 08$ & $81 / 38 / 58$ & ELOISE & 105 \\
\hline 2.85 & & 500 & & $28 / 04 / 10$ & $81 / 45 / 20$ & AUBURNDALE & 105 \\
\hline 4.55 & & 191 & & $28 / 03 / 30$ & $81 / 46 / 03$ & AUBURNDALE & 105 \\
\hline 1.33 & & 437 & 131 & $28 / 03 / 07$ & $81 / 44 / 59$ & WINTER HAVEN & 105 \\
\hline .96 & & 124 & & $28 / 01 / 41$ & $81 / 45 / 54$ & AUBURNDALE & 105 \\
\hline 10.1 & & 333 & 131 & $28 / 01 / 57$ & $81 / 45 / 21$ & AUBURNDALE & 105 \\
\hline $12 \cdot 8$ & & 634 & 131 & $28 / 01 / 20$ & $81 / 44 / 16$ & WINTER HAVEN & 105 \\
\hline 14.9 & & 284 & 131 & $28 / 00 / 25$ & $81 / 44 / 45$ & WINTER HAVEN & 105 \\
\hline 2.19 & & 551 & 131 & $27 / 58 / 18$ & $81 / 40 / 27$ & ELOISE & 105 \\
\hline 5.62 & & 1172 & 131 & $27 / 59 / 34$ & $81 / 41 / 34$ & ELOISE & 105 \\
\hline 22.7 & & 307 & 131 & $27 / 59 / 26$ & $81 / 43 / 48$ & ELOISE & 105 \\
\hline 1.50 & & 488 & 133 & $27 / 58 / 25$ & $81 / 45 / 00$ & ELOISE & 105 \\
\hline 18.0 & & 664 & 100 & $27 / 53 / 48$ & $81 / 43 / 28$ & ELOISE & 105 \\
\hline
\end{tabular}


DRAINAGE AREAS FOR SELECTEO LAKE SITES IN FLORIDA

PEACE, WITHLACOOCHEE, HILLSBOROUGH RIVERS \& WESTERN COASTAL AREAS

PEACE RIVER BASIN

SITE

02294224 LAKE GIBSON NR LAKELAND

02294247 LAKE MORTON AT LAKELAND

02294249 MIRROR LAKE AT LAKELAND

02294254 LAKE BONNY AT LAKELAND

02294259 LAKE PARKER AT LAKELAND

02294269 CRYSTAL LAKE NR LAKELAND

02294298 LAKE ARIETTA NR AUBURNDALE

02294300 LAKE WHISTLER NR AUBURNDALE

02294303 ARIANA LAKE AT AUBURNDALE

02294305 LAKE LENA AT AUBURNDALE

\begin{tabular}{|c|c|c|c|c|c|c|c|c|}
\hline $\begin{array}{l}\text { DRA I NAGE } \\
\text { AREA } \\
\text { SQ.MI. }\end{array}$ & $\begin{array}{l}\text { DRAINAGE. } \\
\text { AREA } \\
\text { QUALIF IER }\end{array}$ & $\begin{array}{l}\text { SURF ACE } \\
\text { AREA } \\
\text { ACRES }\end{array}$ & $\begin{array}{c}A T \\
\text { ELEV }\end{array}$ & $\begin{array}{c}\mathrm{LAT} \\
\mathrm{DG} / \mathrm{MI} / \mathrm{SD}\end{array}$ & $\begin{array}{l}\text { LONG } \\
\mathrm{DG} / \mathrm{MI} / \mathrm{SO}\end{array}$ & QUADRANGLE & NAME & $\begin{array}{l}\text { CNTY } \\
\text { CODE }\end{array}$ \\
\hline 4.31 & & 477 & 145 & $28 / 06 / 40$ & $81 / 57 / 18$ & LAKELAND & & 105 \\
\hline .25 & & 42 & 179 & $28 / 02 / 22$ & $81 / 57 / 08$ & LAKELAND & & 105 \\
\hline .16 & & 19 & 179 & $28 / 02 / 40$ & $81 / 57 / 11$ & LAKELAND & & 105 \\
\hline 2.89 & & 346 & 130 & $28 / 02 / 33$ & $81 / 55 / 41$ & LAKELAND & & 105 \\
\hline 23.6 & & 2291 & 128 & $28 / 02 / 59$ & $81 / 55 / 22$ & LAKELAND & & 105 \\
\hline .40 & & 33 & 131 & $28 / 01 / 30$ & $81 / 54 / 35$ & LAKELAND & & 105 \\
\hline 3.70 & & 764 & & $28 / 05 / 48$ & $81 / 47 / 48$ & AUBURNDALE & & 105 \\
\hline .63 & & 78 & & $28 / 05 / 14$ & $81 / 48 / 46$ & AUBURNDALE & & 105 \\
\hline 7.44 & & 1019 & & $28 / 05 / 13$ & $81 / 47 / 30$ & AUBURNDALE & & 105 \\
\hline .75 & & 208 & & $28 / 03 / 42$ & $81 / 48 / 22$ & AUBURNDALE & & 105 \\
\hline 1.92 & & 356 & 133 & $28 / 01 / 05$ & $81 / 56 / 50$ & LAKELAND & & 105 \\
\hline $14 \cdot 3$ & & 343 & 105 & $27 / 58 / 52$ & $81 / 53 / 50$ & MULBERRY & & 105 \\
\hline 2.52 & & 659 & 131 & $27 / 58 / 35$ & $81 / 46 / 32$ & BARTOW & & 105 \\
\hline 131 & & 4541 & 97 & $27 / 57 / 48$ & $81 / 51 / 29$ & BARTOW & & 105 \\
\hline 11.0 & & 1544 & 132 & $27 / 48 / 30$ & $81 / 40 / 01$ & ALTURAS & & 105 \\
\hline & & & 194 & 42 & 143 & LAKELAN & & 105 \\
\hline
\end{tabular}

MYAKKA RIVER EASIN

$\begin{array}{ccrcccc}\begin{array}{c}\text { DRAINAGE } \\ \text { AREA } \\ \text { SQ.MI. }\end{array} & \begin{array}{c}\text { DRAINAGE } \\ \text { AREA }\end{array} & \begin{array}{c}\text { SURFACE } \\ \text { QUALIFIER }\end{array} & \begin{array}{c}\text { AREA } \\ \text { ACRES }\end{array} & \begin{array}{c}\text { AT } \\ \text { ELEV }\end{array} & \begin{array}{c}\text { LAT } \\ \text { DG/MI/SD }\end{array} & \text { LONG } \\ \text { DG/MI/SD } & \text { QUADRANGLE NAME } & \text { CNTY } \\ 215 & & 933 & 27 / 15 / 50 & 82 / 17 / 20 & \text { OLD MYAKKA } & 115 \\ 240 & & 586 & 27 / 13 / 05 & 82 / 20 / 00 & \text { LOWER MYAKKA LAKE } & 115\end{array}$


SITE
NIJMBER

NAME AND LOCATION

02300400 LAKE WIMAUMA AT WIMAUMA

02319200 LAKE OCTAHATCHEE NR JENNINGS

$$
\begin{aligned}
& \text { SITE } \\
& \text { NIJMBER }
\end{aligned}
$$

NAME AND LOCATION

02300900 SCOTT LAKE NR LAKELAND
NAME AND LOCATION

02302140 LAKE HUNTER AT LAKELAND

02302184 LAKE REULAH AT LAKELANO

02303220 VALRICO LAKE NR VALRICO

02303290 LAKE THONOTOSASSA AT THONOTOSASSA 02303379 KING LAKE NR SAN ANTONIO

02303412 DEANE. LAKE NR DREXEL

02303416 CURVE LAKE NR DREXEL

02303432 . HANNA LAKE NR LUTZ

02303438 KING LAKE AT DREXEL

02303440 LAKE PADGETT NR LUTZ

02303450 EAST LAKE. NR DREXEL

02303610 LAKE KELL NK LUTZ

02303663 KEENE LAKE NR LUTZ

02303700 LAKE STEMPEK NR LUTZ LITTLE MANATEE RIVER BASIN

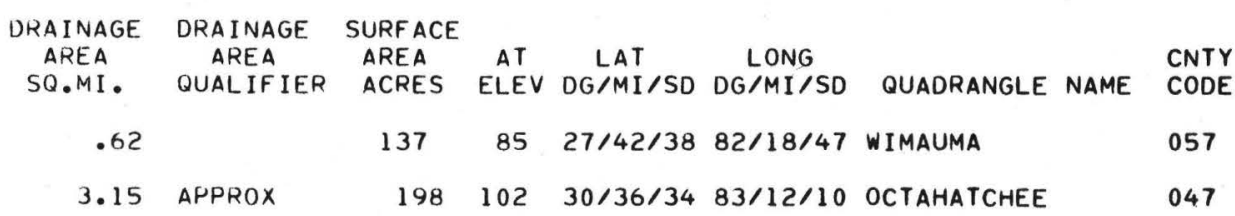

\begin{tabular}{|c|c|c|c|c|c|c|c|c|}
\hline $\begin{array}{l}\text { DRAINAGE } \\
\text { AREA } \\
\text { SQ.MI. }\end{array}$ & $\begin{array}{l}\text { DRAINAGE } \\
\text { AREA } \\
\text { QUALIFIFR }\end{array}$ & $\begin{array}{l}\text { SURFACE } \\
\text { AREA } \\
\text { ACRES }\end{array}$ & $\begin{array}{c}\text { AT } \\
\text { ELEV }\end{array}$ & $\begin{array}{c}\text { LAT } \\
D G / M I / S D\end{array}$ & $\begin{array}{c}\text { LONG } \\
D G / M I / S D\end{array}$ & QUADRANGLE I & NAME & $\begin{array}{l}\text { CNTY } \\
\text { CODE }\end{array}$ \\
\hline .80 & & 100 & 162 & $28 / 01 / 43$ & $81 / 58 / 11$ & LAKELAND & & 105 \\
\hline .12 & & 25 & & $28 / 02 / 24$ & $81 / 58 / 02$ & LAKELAND & & 105 \\
\hline 8.25 & & 130 & 42 & $27 / 57 / 21$ & $82 / 15 / 39$ & BRANDON & & 101 \\
\hline 60 & APPROX & 824 & & $28 / 03 / 33$ & $82 / 17 / 09$ & THONOTOSASSA & & 057 \\
\hline 3.37 & & 263 & 102 & $28 / 17 / 43$ & $82 / 17 / 37$ & SAN ANTONIO & & 101 \\
\hline 1.50 & & 179 & 74 & $28 / 15 / 29$ & $82 / 26 / 14$ & EHREN & & 101 \\
\hline .14 & & 24 & 76 & $28 / 15 / 10$ & $82 / 27 / 42$ & EHREN & & 101 \\
\hline .60 & & 29 & & $28 / 08 / 26$ & $82 / 26 / 52$ & LUTZ & & 057 \\
\hline 1.7 & APPROX & 123 & & $28 / 13 / 46$ & $82 / 27 / 21$ & LUTZ & & 101 \\
\hline 6.6 & APPROX & 200 & & $28 / 12 / 12$ & $82 / 27 / 43$ & LUTZ & & 101 \\
\hline$\cdot 6$ & APPROX & 99 & & $28 / 12 / 52$ & $82 / 26 / 31$ & LUTZ & & 101 \\
\hline 8.9 & APPROX & 31 & & $28 / 09 / 56$ & $82 / 27 / 20$ & LUTZ & & 057 \\
\hline .38 & & 31 & & $28 / 08 / 37$ & $82 / 26 / 48$ & LUTZ & & 057 \\
\hline .74 & & 126 & & $28 / 08 / 09$ & $82 / 27 / 22$ & LUTZ & & 057 \\
\hline
\end{tabular}


DRAINAGE AREAS FOR SELECTED LAKE SITES IN FLORIDA

PEACE, WITHLACOOCHEE, HILLSBOROUGH RIVERS \& WESTERN COASTAL AREAS

SITE NAME AND LOCATION

02304700 LAKE HOBBS NR LUTZ

02304844 COOPER LAKE NR LUTZ

02304949 STRAWBERRY LAKE NR LUTZ

02304951 CRYSTAL LAKE NR LUTZ

02304999 LAKE HUTCHINS NR LUTZ

02305175 LAKE CRENSHAW NR LUTZ

02305178 SADDLEBACK LAKE NR LUTZ

02305200 ROUND LAKE NR LUTZ

02305273 BRANT LAKE NR LUTZ

02305288 LAKE CHARLES NR LUTZ

HILLSBOROUGH RIVER BASIN

02305342 PLATT LAKE NR LUTZ

02305434 CHAPMAN LAKE NR LUTZ
NAME AND LOCATION

02306200 LAKE MAGDALENE NR LUTZ

02306300 BAY LAKE NR SULPHUR SPRINGS

02306371 LAKE ELLEN NR SULPHUR SPRINGS

02306451 LAKE LIPSEY NR SULPHUR SPRINGS

02306600 LAKE CARROLL NR SULPHUR SPRINGS

02306614 WHITE TROUT LAKE NR SULPHUR SPRINGS

02306700 BROWNS LAKE NR LUTZ

02306704 LAKE HARVEY NR LUTZ

02306711 LAKE THOMAS NR LUTZ

\begin{tabular}{|c|c|c|c|c|c|c|c|c|}
\hline $\begin{array}{l}\text { DRAINAGE } \\
\text { AREA } \\
\text { SQ.MI. }\end{array}$ & $\begin{array}{l}\text { DRA INAGE } \\
\text { AREA } \\
\text { QUALIF IER }\end{array}$ & $\begin{array}{l}\text { SURFACE } \\
\text { AREA } \\
\text { ACRES }\end{array}$ & $\begin{array}{c}\text { AT } \\
\text { ELEV }\end{array}$ & $\begin{array}{c}\text { LAT } \\
\mathrm{DG} / \mathrm{MI} / \mathrm{SD}\end{array}$ & $\begin{array}{c}\text { LONG } \\
\mathrm{DG} / \mathrm{MI} / \mathrm{SD}\end{array}$ & QUADRANGLE & NAME & $\begin{array}{l}\text { CNTY } \\
\text { CODE }\end{array}$ \\
\hline . 92 & & 66 & & $28 / 09 / 30$ & $82 / 28 / 00$ & LUTZ & & 057 \\
\hline 1.7 & APPROX & 80 & & $28 / 08 / 55$ & $82 / 28 / 20$ & LUTZ & & 057 \\
\hline 1.9 & APPROX & 39 & & $28 / 08 / 12$ & $82 / 28 / 30$ & LUTZ & & 057 \\
\hline 2.1 & APPROX & 21 & & $28 / 07 / 59$ & $82 / 28 / 40$ & LUTZ & & 057 \\
\hline 2.7 & APPROX & 21 & & $28 / 07 / 40$ & $81 / 29 / 15$ & LUTZ & & 057 \\
\hline 1.3 & APPROX & 33 & & $28 / 07 / 36$ & $82 / 29 / 41$ & LUTZ & & 057 \\
\hline 1.5 & APPROX & 34 & 54 & $28 / 07 / 06$ & $82 / 29 / 45$ & SULPHUR SPGS & & 057 \\
\hline .07 & & 9 & & $28 / 07 / 16$ & $82 / 30 / 04$ & CITRUS PARK & & 057 \\
\hline .7 & APPROX & 56 & 58 & $28 / 07 / 36$ & $82 / 28 / 27$ & LUTZ & & 057 \\
\hline .08 & & 15 & 53 & $28 / 06 / 53$ & $82 / 28 / 57$ & SULPHUR SPGS & & 057 \\
\hline 9.2 & APPROX & 63 & 49 & $28 / 05 / 39$ & $82 / 28 / 55$ & SULPHUR SPGS & & 057 \\
\hline 1.9 & APPROX & 42 & 51 & $28 / 06 / 09$ & $82 / 27 / 48$ & SULPHUR SPGS & & 057 \\
\hline
\end{tabular}

TAMPA BAY AND COASTAL AREAS

\begin{tabular}{|c|c|c|c|c|c|c|c|c|}
\hline $\begin{array}{l}\text { DRAINAGE } \\
\text { AREA } \\
\text { SQ.MI. }\end{array}$ & $\begin{array}{l}\text { DRA INAGE } \\
\text { ARE.A } \\
\text { QUAL IF IER }\end{array}$ & $\begin{array}{l}\text { SURFACE } \\
\text { AREA } \\
\text { ACRES }\end{array}$ & $\begin{array}{c}\text { AT } \\
\text { ELEV }\end{array}$ & $\begin{array}{c}\text { LAT } \\
\mathrm{DG} / \mathrm{MI} / \mathrm{SD}\end{array}$ & $\begin{array}{c}\text { LONG } \\
\mathrm{DG} / \mathrm{MI} / \mathrm{SD}\end{array}$ & QUADRA & NGLE NAME & $\begin{array}{l}\text { CNTY } \\
\text { CODE }\end{array}$ \\
\hline 1.67 & & 232 & & $28 / 04 / 55$ & $82 / 29 / 10$ & SULPHUF & SPGS & 057 \\
\hline 2.52 & & 37 & 45 & $28 / 04 / 10$ & $83 / 30 / 00$ & SULPHUF & SPGS & 057 \\
\hline 4.18 & & 50 & 39 & $28 / 03 / 44$ & $82 / 29 / 57$ & SULPHUF & SPGS & 057 \\
\hline .16 & & 22 & 39 & $28 / 03 / 12$ & $82 / 29 / 46$ & SULPHUR & SPGS & 057 \\
\hline 1.66 & & 188 & 34 & $28 / 03 / 20$ & $82 / 28 / 55$ & SULPHUF & SPGS & 057 \\
\hline 2.1 & APPROX & 75 & 33 & $28 / 02 / 31$ & $82 / 29 / 34$ & SULPHUR & SPGS & 057 \\
\hline 1.3 & АРPROX & 29 & & $28 / 10 / 19$ & $82 / 29 / 01$ & LUTZ & & 101 \\
\hline 1.7 & APPROX & 21 & & $28 / 0 y / 43$ & $82 / 29 / 12$ & LUTZ & & 057 \\
\hline 1.4 & APPROX & 62 & & $28 / 08 / 47$ & $82 / 29 / 21$ & LUTZ & & 057 \\
\hline
\end{tabular}


SITE

NIIMBER NAMF. AND LOCATION

02306714 LAKE $\triangle$ LLEN NR LUTZ

02306723 TURKEY FORD LAKE NR LUTZ

02306744 PRETTY LAKE NR CITRUS PAKK

02306782 VAN DYKF. LAKE NR LUTZ

02306788 DOSSON LAKE NK LUT7

02306822 UNNAMED LAKE AT LAKE PARK NK LUTZ

02307025 GLASS LAKE NR LAKE FEPN

02307197 MOUNO LAKE NR ODESSA

02307227 CALM LAKE NK ODESSA

02307242 KEYSTONE LAKE NR ODESSA

02307283 CKESCENT LAKE NR ONESSA

02307295 ISLAND FORD LAKE NR ODESSA

02307311 SUNSFT LAKE NR LAKF FFRN

02307328 LAKE ALICE NR OCFSSA

02307331 LAKE TAYLOR. NR ONESSA

023073 66 LAKE JUANITA NR CITRUSS PARK

02307369 RAINBON LAKE NR CITRUS PARK

02307378 CHUKCH LAKE NR CITRUS PARK

02307384 ECHO LAKE NK CITRUS PARK

02307388 BUICK LAKE NH LAKE FERN

02307479 LAKE TARPON NR TARPON: SPRINGS

02307696 ALLIGATOR LAKE AT SAFETY HARFOR

02308000 SAWGRASS LAKE NR PINELLAS PARK

02308293 LAKE MAGGIORE AT ST PETERSBURG

\begin{tabular}{|c|c|c|}
\hline $\begin{array}{l}\text { DRAIVAGE } \\
\text { AREA } \\
\text { SO.MI. }\end{array}$ & $\begin{array}{l}\text { DRA INAGE } \\
\text { AKEA } \\
\text { QUALIF IER }\end{array}$ & $\begin{array}{l}\text { SURFACE } \\
\text { AREA } \\
\text { ACRES }\end{array}$ \\
\hline 1.8 & APPROX & 28 \\
\hline 9.8 & APPROX & 93 \\
\hline 13 & APPROX & 79 \\
\hline .4 & APPROX & 13 \\
\hline .7 & APPROX & 10 \\
\hline .1 & APPROX & 9 \\
\hline .23 & & 17 \\
\hline .67 & & 78 \\
\hline .4 & APPROX & 125 \\
\hline 10 & APPROX & 388 \\
\hline 1.2 & APPROX & 46 \\
\hline 12 & APPROX & 96 \\
\hline$\cdot 26$ & & 36 \\
\hline .5 & APPROX & 94 \\
\hline 1.0 & APPROX & 44 \\
\hline .5 & $\triangle P P R O X$ & 24 \\
\hline - 8 & APFROX & 48 \\
\hline .51 & & 67 \\
\hline .89 & & 25 \\
\hline .55 & & 34 \\
\hline $60 \cdot 0$ & & 2534 \\
\hline 9.00 & & 76 \\
\hline 3.97 & & 21 \\
\hline 4.83 & & 380 \\
\hline
\end{tabular}

AT LAT LONG CNTY DG/MI/SD DG/MI/SD QUADRANGLE NAME CODE 28/09/24 82/29/13 LUTZ 28/08/08 82/32/24 ODESSA 057 057 28/06/16 82/33/55 CITRUS PARK 057 28/07/43 82/31/09 ODESSA 057 54 28/07/27 82/31/32 CITRUS PARK 057 $28 / 07 / 20 \quad 82 / 30 / 44$ CITRUS PARK 057

31 28/05/57 82/37/08 CITRUS PARK 057 28/08/54 82/34/31 ODESSA 057

$48 \quad 28 / 08 / 20 \quad 82 / 35 / 00$ ODESSA 057 $\begin{array}{lll}28 / 08 / 50 & 82 / 35 / 40 & \text { ODESSA }\end{array}$ 28/09/37 82/35/42 ODESSA 057 $\begin{array}{llll}28 / 09 / 01 & 82 / 35 / 41 & \text { ODESSA } & 057\end{array}$ 28/08/02 82/37/28 ELFERS 057 $28 / 08 / 0182 / 36 / 07$ ODESSA 057 28/08/13 82/36/44 ODESSA 057 28/06/56 82/35/23 CITRUS PARK 057 28/06/51 82/35/45 CITRUS PARK 057 28/06/15 82/36/00 CITRUS PARK 057 28/06/22 82/36/12 CITRUS PARK 057 28/06/31 82/37/16 CITRUS PARK 057 28/05/35 82/43/20 OLDSMAR 103 5 27/58/45 82/41/45 SAFETY HARBOR 103

$927 / 50 / 33 \quad 82 / 39 / 48$ ST PETERSBURG 103 27/44/32 82/39/23 PASS-A-GRILLE BCH 103 
DRAINAGE AREAS FOR SELECTED LAKE SITES IN FLORIDA

PEACE, WITHLACOOCHEE, HILLSHOROUGH RIVERS \& WESTERN COASTAL AREAS

COASTAL AREA FROM TAMPA BAY TO WITHL.ACOOCHEE RIVER

SITE
NIJMBER

NAME ANID LOCATION

02308888 SEMINOLF LAKE NR LAKGO

02309584 LAKE THOMAS AT DREXEL

02309765 LINUA LAKE. AT DENHAM

02309814 CAMP LAKE NR UENHAM

02309869 BLACK LAKE INR ODESSA

02309872 PARKER LAKE INR ODESSA

02310210 HORSE LAKE NR BKONKSVILLE

02310220 NEFF LAKE NR BROOKSVILLE

02310227 CREWS LAKE. NR LOYCE -NIDRTH-

02310230 LAKE IOLA NR SAN ANTONIO

02310260 CKE WS LAKE NR LOYCF - SOUTH-

02310290 MOON LAKE NR NEW PORT RICHEY

02310400 HUNTERS LAKE NR AKIPEKA

02310520 HIGHLAND LAKE NR BROOKSVILLE

02310680 MORRISON POND AT LECANTO

SITE
NUMBER

NAME AND LOCATION

02310760 LAKE JULIANA NR POLK CITY

02310780 LAKE MATTIE NR POLK CITY

02310850 LAKE HELENE. NR POLK CITY

02310950 LAKE DEESON NR LAKELLAND

02311500 CLEAR LAKE AT SAN ANTONIO

\begin{tabular}{|c|c|c|c|c|c|c|c|c|}
\hline $\begin{array}{l}\text { DRAINAGE } \\
\text { AREA } \\
\text { SQ.MI. }\end{array}$ & $\begin{array}{l}\text { DRAINAGE } \\
\text { AREA } \\
\text { QUALIFIER }\end{array}$ & $\begin{array}{l}\text { SURFACE } \\
\text { AREA } \\
\text { ACRES }\end{array}$ & $\begin{array}{l}\text { AT } \\
\text { ELEV }\end{array}$ & $\begin{array}{c}\text { LAT } \\
D G / M I / S D\end{array}$ & $\begin{array}{c}\text { LONG } \\
\mathrm{DG} / \mathrm{MI} / \mathrm{SD}\end{array}$ & QUADRANGLE & NAME & $\begin{array}{l}\text { CNTY } \\
\text { CODE }\end{array}$ \\
\hline 6.94 & & 684 & 5 & $27 / 50 / 20$ & $82 / 46 / 50$ & CLEAR'WATER & & 103 \\
\hline 1.0 & ARPKUXX & 162 & & $28 / 14 / 14$ & $82 / 28 / 08$ & LUTZ & & 101 \\
\hline .94 & & 31 & & $28 / 11 / 14$ & $82 / 28 / 52$ & LUTZ & & 101 \\
\hline 1.0 & APPROX & 20 & & $28 / 11 / 03$ & $82 / 29 / 26$ & LUTZ & & 101 \\
\hline .3 & APPROX & 5 & & $28 / 11 / 22$ & $82 / 34 / 36$ & ODESSA & & 101 \\
\hline 3.09 & & 94 & & $28 / 10 / 40$ & $82 / 34 / 46$ & ODESSA & & 101 \\
\hline 7.74 & & 21 & & $28 / 32 / 15$ & $82 / 25 / 19$ & BROOKSVILLE & & 053 \\
\hline 5.08 & & 224 & 101 & $28 / 28 / 45$ & $82 / 19 / 15$ & SPRING LAKE & & 053 \\
\hline 138 & & & & $28 / 24 / 04$ & $82 / 30 / 13$ & PORT RICHEY & NE & 101 \\
\hline .49 & & 104 & & $28 / 23 / 28$ & $82 / 17 / 54$ & SPRING LAKE & & 101 \\
\hline 43 & APPROX & 10 & 65 & $28 / 23 / 05$ & $82 / 29 / 17$ & MASARYKTOWN & & 051 \\
\hline 138 & & 749 & & $28 / 23 / 30$ & $82 / 30 / 40$ & PORT RICHEY & $\mathrm{NE}$ & 101 \\
\hline .37 & & 98 & 41 & $28 / 17 / 15$ & $82 / 36 / 49$ & FIVAY JUNCTI & I ON & 101 \\
\hline 2.37 & & 301 & 19 & $28 / 26 / 40$ & $82 / 37 / 40$ & ARIPEKA & & 053 \\
\hline 1.22 & & 50 & & $28 / 32 / 50$ & $82 / 33 / 48$ & WEEKI WACHEE & E SPGS & 053 \\
\hline 16.2 & & 1 & & $28 / 51 / 26$ & $82 / 29 / 04$ & LECANTO & & 017 \\
\hline
\end{tabular}

WITHLACOOCHEE RIVER BASIN

\begin{tabular}{|c|c|c|c|c|c|c|c|c|}
\hline $\begin{array}{l}\text { DRAI VAGE } \\
\text { AREA } \\
\text { SQ.MI. }\end{array}$ & $\begin{array}{l}\text { DRAINAGE } \\
\text { AREA } \\
\text { QUALIF IER }\end{array}$ & $\begin{array}{l}\text { SURFACE } \\
\text { AREA } \\
\text { ACRES }\end{array}$ & $\begin{array}{l}\text { AT } \\
\text { ELEV }\end{array}$ & $\begin{array}{c}\text { LAT } \\
D G / M I / S D\end{array}$ & $\begin{array}{l}\text { LONG } \\
D G / M I / S D\end{array}$ & QUADRANGLE & NAME & $\begin{array}{l}\text { CNTY } \\
\text { CODE }\end{array}$ \\
\hline 5.4 & APPROX & 92 & 132 & $28 / 07 / 51$ & $81 / 47 / 45$ & POLK CITY & & 105 \\
\hline 14.7 & & 1813 & 132 & $28 / 07 / 34$ & $81 / 46 / 56$ & POLK CITY & & 105 \\
\hline .42 & & 54 & 140 & $28 / 10 / 25$ & $81 / 48 / 21$ & POLK CITY & & 105 \\
\hline .96 & & 116 & 134 & $28 / 06 / 37$ & $81 / 55 / 51$ & LAKELAND & & 105 \\
\hline .92 & & 158 & 127 & $28 / 20 / 20$ & $82 / 16 / 02$ & SAN ANTONIO & & 101 \\
\hline
\end{tabular}


DRAINAGE AREAS FUR SELECTEU LAKE SITES IN FLORIDA

PEACE, WITHLACOOCHEE, HILLSBOROUGH RIVERS \& WESTERN COASTAL AREAS WITHLACOOCHEE RIVER BASIN

SITE

NUMBER
NAME AND LOCATION

02312100 SPRING LAKE NR BROOKSVILLE

02312520 LAKE LINDSEY INR BROOKSVILLE

02312670 LAKE CATHERINE AT GROVELAND

02312688 LAKE DEATON NR WILDWOOD

02312691 LAKE OKAHUMPKA NR WILDWOON

02312694 LADY LAKE NR LADY LAKE

02312696 LAKE MIONA NR OXFORO

02312698 LAKE PANASOFFKEE NR PANASOFFKEE

02312794 LITTLF LAKE AT FLORAL CITY

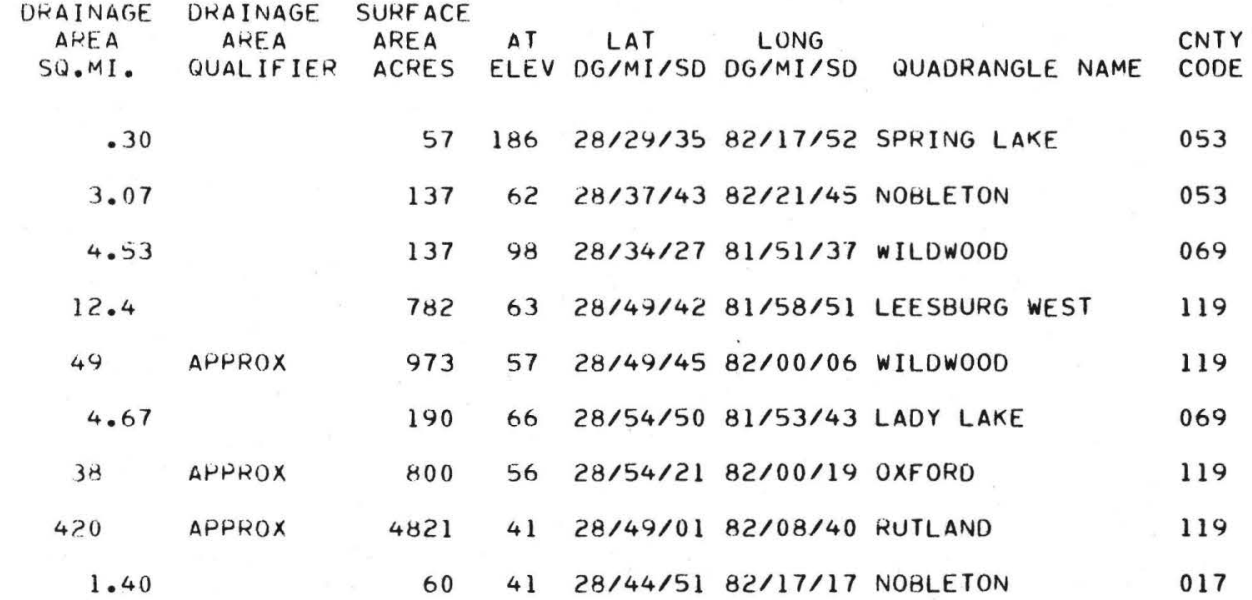

SUWANNEE AND AUCILLA RIVERS

\author{
SITE \\ NUMBER \\ NAME AND LOCATION
}

02313510 CHUNKY POND NR BRONSON

\begin{tabular}{|c|c|c|c|c|c|c|c|c|}
\hline DRAINAGE & DRA INAGE & SURFACE & & & & & & \\
\hline $\begin{array}{c}\text { AREA } \\
\text { SQ.MI. }\end{array}$ & $\begin{array}{c}\text { AREA } \\
\text { QUALIFIER }\end{array}$ & $\begin{array}{l}\text { AREA } \\
\text { ACRES }\end{array}$ & $\begin{array}{l}\text { AT } \\
\text { ELEV }\end{array}$ & $\begin{array}{c}\text { LAT } \\
D G / M I / S D\end{array}$ & $\begin{array}{c}\text { LONG } \\
D G / M I / S D\end{array}$ & QUADRANGLE & NAME & $\begin{array}{l}\text { CNTY } \\
\text { CODE }\end{array}$ \\
\hline 23 & APPROX & 650 & 55 & $29 / 23 / 36$ & $82 / 37 / 19$ & BRONSON NE & & 075 \\
\hline
\end{tabular}

COASTAL AREA BETWEE SUN SUNANEE AND AUCILLA RIVERS

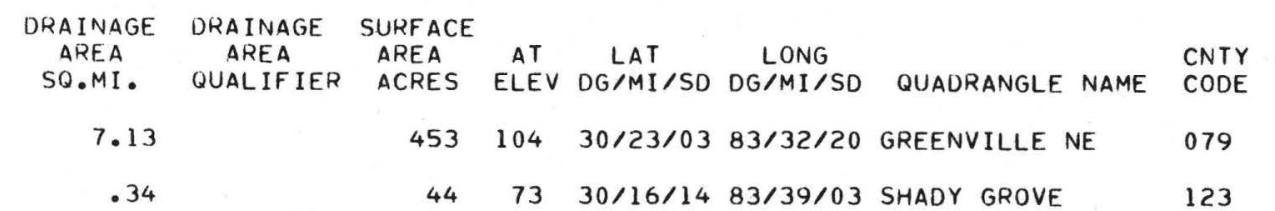

.34
SITE
NUMBER

NAME AND LOCATION

02325700 SAMPALA LAKE NR GREENVILLE

02325820 ANDREWS LAKE NEAR SHADY GROVE 
SITE
NUMBER NAME AND LOCATION

02319150 CHERRY LAKE NR CHERRY LAKE AREA AREA AREA AT LAT LONG

AREA
SQ.MI.

$$
1.58
$$

SUWANNEE RIVER BASIN BELOW WITHLACOOCHE. RIVER EXCLUDING SANTA FE RIVER BASIN

SI TE
NUMBER

NAME AND LOCATION

02320200 TOWNSEND POND NR MAYO

02320275 LOW LAKE NR WELLBORN

02323300 GOVERNOR HILL LAKE NR OLD TOWN

$\begin{array}{ccrllll}\begin{array}{c}\text { DRAINAGE } \\ \text { AREA }\end{array} & \begin{array}{c}\text { DRAINAGE } \\ \text { AREA }\end{array} & \begin{array}{c}\text { SURFACE } \\ \text { AREA }\end{array} & \text { AT } & \text { LAT } & \text { LONG } & \\ \text { SQ.MI } & \text { QUALIFIER } & \text { ACRES } & \text { ELEV } & \text { DG/MI/SD } \\ \text { DG/MI/SD } & \text { QUADRANGLE NAME } & \text { CNTY } \\ 6.40 & 111 & 71 & 30 / 02 / 27 & 83 / 07 / 11 & \text { MAYO SE } & 067 \\ 2.89 & 69 & 46 & 30 / 13 / 16 & 82 / 50 / 14 & \text { WELLBORN } & 121 \\ 1.61 & 153 & 52 & 29 / 45 / 08 & 83 / 02 / 18 & \text { MALLORY SWAMP SE } & 029\end{array}$

SANTA FE. RIVER EASIN

\author{
SI TE
NUMBER \\ NAME AND LOCATION
}

02320600 SANTA FE LAKE NR KEYSTONE HEIGHTS

02320630 LAKE ALTHO AT WALDO

02320750 LAKE SAMPSON NEAR STARKE

02321300 LAKE BUTLER AT LAKE BUTLER

02322550 WATERS LAKE NR TRENTON

02322600 ALLIGATOR LAKE AT LAKE. CITY

\begin{tabular}{|c|c|c|c|c|c|c|c|}
\hline $\begin{array}{l}\text { DRAINAGE } \\
\text { AREA } \\
\text { SQ.MI. }\end{array}$ & $\begin{array}{l}\text { DRAINAGE } \\
\text { AREA } \\
\text { QUALIFIER }\end{array}$ & $\begin{array}{l}\text { SURFACE } \\
\text { AREA } \\
\text { ACRES }\end{array}$ & $\begin{array}{l}\text { AT } \\
\text { ELEV }\end{array}$ & $\begin{array}{c}\text { LAT } \\
D G / M I / S D\end{array}$ & $\begin{array}{c}\text { LONG } \\
\mathrm{DG} / \mathrm{MI} / \mathrm{SO}\end{array}$ & QUADRANGLE NAME & $\begin{array}{l}\text { CNTY } \\
\text { CODE }\end{array}$ \\
\hline $24 \cdot 3$ & & 5299 & 141 & $29 / 45 / 38$ & $82 / 04 / 30$ & KEYSTONE HEIGHTS & 007 \\
\hline 3.39 & & 555 & 141 & $29 / 47 / 19$ & $82 / 09 / 41$ & WALDO & 001 \\
\hline 59.3 & & 2071 & 35 & $29 / 56 / 11$ & $82 / 10 / 15$ & SAMPSON & 007 \\
\hline 3.94 & & 437 & 132 & $30 / 01 / 38$ & $82 / 20 / 18$ & LAKE BUTLER & 125 \\
\hline 15.7 & & 183 & 71 & $29 / 42 / 31$ & $82 / 43 / 59$ & WATERS LAKE & 041 \\
\hline 15.4 & & 348 & 96 & $30 / 10 / 31$ & $82 / 37 / 55$ & LAKE CITY WEST & 023 \\
\hline
\end{tabular}

OCHLOCKONEE RIVER

ST. MARKS AND WAKULLA RIVERS AND COASTAL AREA BETWEEN AUCILLA AND OCHLOCKONEE RIVERS

\author{
SITE \\ NAME AND LOCATION
}

02326600 LAKE MICCOSUKEE NR MICCOSUKEE 02327010 LAKE RRADFORD NR TALLAHASSEE

\begin{tabular}{|c|c|c|c|c|c|c|c|c|}
\hline $\begin{array}{l}\text { DRAINAGE } \\
\text { AREA } \\
\text { SQ.MI. }\end{array}$ & $\begin{array}{l}\text { DRA INAGE } \\
\text { AREA } \\
\text { QUALIFIER }\end{array}$ & $\begin{array}{l}\text { SURFACE } \\
\text { AREA } \\
\text { ACRES }\end{array}$ & $\begin{array}{l}\text { AT } \\
\text { ELEV }\end{array}$ & $\begin{array}{c}\text { LAT } \\
\mathrm{DG} / \mathrm{MI} / \mathrm{SD}\end{array}$ & $\begin{array}{c}\text { LONG } \\
\mathrm{DG} / \mathrm{MI} / \mathrm{SO}\end{array}$ & QUADRANGLE & NAME & $\begin{array}{l}\text { CNTY } \\
\text { CODE }\end{array}$ \\
\hline 240 & & 6312 & 80 & $30 / 36 / 14$ & $84 / 00 / 15$ & MICCOSUKEE & & 065 \\
\hline 20.6 & & 182 & 31 & $30 / 24 / 10$ & $84 / 20 / 05$ & TALLAHASSEE & & 073 \\
\hline
\end{tabular}


DRAINAGE AREAS FOR SELECTED LAKE SITES IN FLORIDA

SI TE
NUMBER

NAME AND LOCATION

02310100 DAN LAKE NR ODESSA

02327150 OTTER LAKE NR PANACEA

02328799 LAKE IAMONIA NR BRADFORDVILLE.

02329200 LAKE JACKSON NR TALLAHASSEE

SITE

NUMBER

NAME AND LOCATION

02358240 OCHEESEE POND NR SNEADS

02358715 LAKE MYSTIC NR BRISTOL
NAME AND LOCATION

02359020 MOSSY POND NR FOUNTAIN

02359100 DEAD LAKE NR WEWAHITCHKA

02359101 DEAD LAKE OUTLET AT WEWAHITCHKA OCHLOCKONEE RIVE

OCHLOCKONEE RIVER BASIN

\begin{tabular}{|c|c|c|c|c|c|c|c|c|}
\hline $\begin{array}{l}\text { DRAINAGE } \\
\text { AREA } \\
\text { SQ.MI. }\end{array}$ & $\begin{array}{l}\text { DRA INAGE } \\
\text { AREA } \\
\text { QUALIF IER }\end{array}$ & $\begin{array}{l}\text { SURFACE } \\
\text { AREA } \\
\text { ACRES }\end{array}$ & $\begin{array}{c}A T \\
\text { ELEV }\end{array}$ & $\begin{array}{c}\text { LAT } \\
D G / M I / S D\end{array}$ & $\begin{array}{c}\text { LONG } \\
D G / M I / S O\end{array}$ & QUADRANGLE & NAME & $\begin{array}{l}\text { CNTY } \\
\text { CODE }\end{array}$ \\
\hline .60 & APPROX & 37 & & $28 / 10 / 00$ & $82 / 38 / 55$ & ELFERS & & 057 \\
\hline 17.9 & & 141 & 4 & $30 / 01 / 32$ & $84 / 25 / 06$ & SOPCHOPPY & & 129 \\
\hline 101 & & 5680 & 98 & $30 / 39 / 04$ & $84 / 12 / 30$ & BEACHTON & & 073 \\
\hline 43.1 & & 4001 & 87 & $30 / 31 / 43$ & $84 / 21 / 30$ & LAKE JACKSON & & 073 \\
\hline
\end{tabular}

APALACHICOLA, CHATTAHOOCHEE AND FLINT RIVERS

APALACHICOLA RIVER

$\begin{array}{ccc}\text { DRAINAGE } & \text { DRAINAGE } & \text { SURFACE } \\ \text { AREA } & \text { AREA } & \text { AREA }\end{array}$

AT LAT LONG

ACRES ELEV DG/MI/SD DG/MI/SO QUADRANGLE NAME CODE

$\begin{array}{llllll}24.2 & 2230 & 111 & 30 / 41 / 34 & 84 / 59 / 26 & \text { SNEADS }\end{array}$

1.04

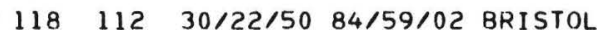

077

CHIPOLA RIVER BASIN

\begin{tabular}{|c|c|c|c|c|c|c|c|c|}
\hline $\begin{array}{l}\text { DRAINAGE } \\
\text { AREA } \\
\text { SQ.MI. }\end{array}$ & $\begin{array}{l}\text { DRAINAGE } \\
\text { AREA } \\
\text { QUALIF IER }\end{array}$ & $\begin{array}{l}\text { SURFACE } \\
\text { AREA } \\
\text { ACRES }\end{array}$ & $\begin{array}{l}\text { AT } \\
\text { ELEV }\end{array}$ & $\begin{array}{c}\text { LAT } \\
D G / M I / S D\end{array}$ & $\begin{array}{c}\text { LONG } \\
D G / M I / S D\end{array}$ & QUADRANGLE & NAME & $\begin{array}{l}\text { CNTY } \\
\text { CODE }\end{array}$ \\
\hline .74 & & 105 & 192 & $30 / 30 / 53$ & $85 / 18 / 36$ & ALFORD SE & & 016 \\
\hline 1,206 & & 3663 & 14 & $30 / 11 / 40$ & $85 / 11 / 50$ & DEAD LAKE & & 045 \\
\hline 1,206 & & 3663 & 14 & $30 / 11 / 40$ & $85 / 11 / 50$ & DEAD LAKE & & 045 \\
\hline
\end{tabular}


DRAINAGE AREAS FOR SELECTEO LAKE SITES IN FLORIDA CHOCTAWHATCHEE, YELLOW. AND ESCAMBIA RIVERS

ST. ANDREW BAY, INFLOW AND COASTAL AREA

SITE

NIMMBER

NAME AND LOCATION

02.359614 GAP LAKE NR GREENHEAD

02.359618 PORTER LAKE NR GREFNHEAD

02359630 HAMMOCK LAKE NR GREENHEAD

02359640 WAGES POND NR GREENHEAD

02359645 GULLY LAKE NR GREENHEAD

02359670 MERIAL LAKE NR GREENHEAD

SITE
NUMBER

NAME AND LOCATION

$$
\begin{aligned}
& \text { DRAINAGE DRA } \\
& \text { AREA }
\end{aligned}
$$

SQ.MI AREA AREA

$$
3.89
$$

7.64

2.22

41.2

43.9

1.57

YELLOW RIVER BASIN
CNTY
CODE

133

$92970 \quad 30 / 29 / 5085 / 33 / 05$ BENNETT 133

$9115130 / 28 / 2585 / 35 / 05$ BENNETT 133

$100 \quad 30 / 30 / 0585 / 35 / 55$ GAP LAKE 133

$17130 / 29 / 3085 / 36 / 55$ BENNETT 133

$22150 \quad 30 / 23 / 10 \quad 85 / 40 / 35$ CRYSTAL LAKE 005

PEA RIVER

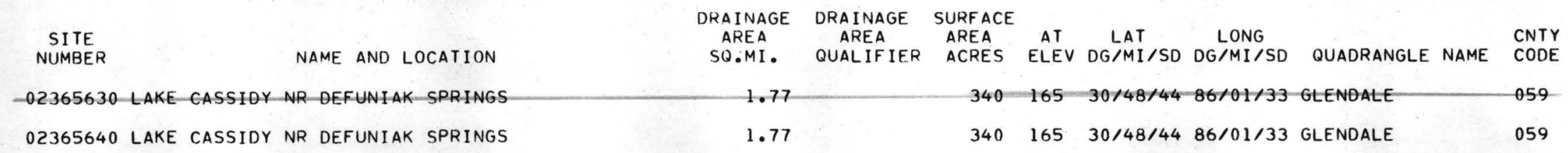

CHOCTAWHATCHEE RIVER BELOW PEA RIVER

SITE
NUMBER

NAME AND LOCATION

02365540 PATE LAKE NR VERNON

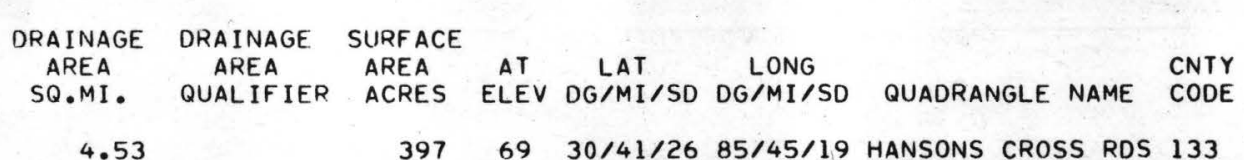

DRAINAGE DRAINAGE SURFACE

4.53

$3976930 / 41 / 26 \quad 85 / 45 / 19$ HANSONS CROSS RDS 133 


\section{REFERENCES}

Conover, C. S., and Leach, S. D., 1975, River basin and hydrologic unit map of Florida: Florida Bureau of Geology Map Series No. 72, 1 map sheet.

Federal Interagency River Basin Committee, Notes on hydrologic activities bulletin No. 4, November 1951: Inter-Agency Coordination of Drainage Area Data.

National Bureau of Standards, Federal Information Processing Standards Publication, FIPS 5-1, and FIPS 6-1. 